\title{
Distinguishability measures between ensembles of quantum states
}

\author{
Ognyan Oreshkov and John Calsamiglia
}

\begin{abstract}
Grup de Física Teòrica, Universitat Autònoma de Barcelona, 08193 Bellaterra (Barcelona), Spain
\end{abstract}
(Dated: June 4, 2018)

\begin{abstract}
A quantum ensemble $\left\{\left(p_{x}, \rho_{x}\right)\right\}$ is a set of quantum states each occurring randomly with a given probability. Quantum ensembles are necessary to describe situations with incomplete a priori information, such as the output of a stochastic quantum channel (generalized measurement), and play a central role in quantum communication. In this paper, we propose measures of distance and fidelity between two quantum ensembles. We consider two approaches: the first one is based on the ability to mimic one ensemble given the other one as a resource and is closely related to the Monge-Kantorovich optimal transportation problem, while the second one uses the idea of extendedHilbert-space (EHS) representations which introduce auxiliary pointer (or flag) states. Both types of measures enjoy a number of desirable properties. The Kantorovich measures, albeit monotonic under deterministic quantum operations, are not monotonic under generalized measurements. In contrast, the EHS measures are. We present operational interpretations for both types of measures. We also show that the EHS fidelity between ensembles provides a novel interpretation of the fidelity between mixed states - the latter is equal to the maximum of the fidelity between all pure-state ensembles whose averages are equal to the mixed states being compared. We finally use the new measures to define distance and fidelity for stochastic quantum channels and positive operator-valued measures (POVMs). These quantities may be useful in the context of tomography of stochastic quantum channels and quantum detectors.
\end{abstract}

\section{INTRODUCTION}

A fundamental difference between classical and quantum systems is that, while classical states can be faithfully distinguished, two generic quantum states cannot be distinguished with arbitrary precision by any operational means. A natural measure that quantifies the similarity of two pure quantum states $|\psi\rangle$ and $|\phi\rangle$ is the transition probability between them, i.e., the probability with which the two states would yield the same outcome under a measurement for which one of the states is the unique state that yields a particular outcome with certainty. This quantity is symmetric with respect to the states and is given by the square of their overlap, $|\langle\psi \mid \phi\rangle|^{2}$. In the case of mixed states, there is no straightforward analogue of the transition probability since there is no measurement for which a mixed state is the unique state that yields a particular outcome with certainty.

A generalization of the concept of transition probability to mixed states was proposed by Uhlmann [1] and it is given by the minimum of the transition probability between two purifications of the mixed states, over all possible purifications. The square root of this quantity, which is given by the simple expression

$$
F(\rho, \sigma)=\operatorname{Tr} \sqrt{\sqrt{\sigma} \rho \sqrt{\sigma}}
$$

is known as the square root fidelity between two density matrices $\sigma$ and $\rho$ and has proven extremely useful in quantum information theory [2]. From the square root fidelity (or fidelity for short), one can define various distances between states, such as the Bures distance $B(\rho, \sigma)=\sqrt{1-F(\rho, \sigma)}$ 3, 4] or the Bures angle $A(\rho, \sigma)=\arccos F(\rho, \sigma)$ [2, 5], which can be regarded as measures of the difference between two states.
In addition to fidelity-based measures, various other measures of distance have been proposed (see, e.g., Refs. [6, 7, 8, 9, 10, 11]). The trace distance [6],

$$
\Delta(\rho, \sigma)=\frac{1}{2}\|\rho-\sigma\|
$$

for example, where $\|O\|=\operatorname{Tr} \sqrt{O^{\dagger} O}$ is the trace norm of an operator $O$, is widely used due to its simple form, various useful properties, and its operational meaning related to the maximum probability with which the two states $\rho$ and $\sigma$ can be distinguished by a measurement.

The problem of distinguishing two quantum states bares strong similarity to the problem of distinguishing two classical probability distributions by looking at the value of a random variable sampled from one of them. Unless the supports of the two distributions have no overlap, the probability of guessing correctly from which ensemble the variable was drawn is strictly smaller than unity. In the classical case, however, the two probability distributions concern the outcomes of only a single observable - the one corresponding to the random variable. In the quantum case, there is a continuum of possible observations that one can perform on the systems and a continuum of corresponding random variables.

Different quantum measurements establish different correspondence between quantum states and probability distributions. This suggests a natural approach to defining distinguishability measures between states. For instance, the fidelity between two quantum states is equal to the minimum statistical overlap between the probability distributions generated by all possible measurements performed on the states [12]. The statistical overlap in question is the Bhattacharyya coefficient $\sum_{x} \sqrt{P(x) Q(x)}$ between classical probability distribu- 
tions $P(x)$ and $Q(x)$ (here $x$ is a classical random variable). Similarly, the trace distance (2) can be obtained by maximizing over all possible measurements the Kolmogorov distance $\sum_{x} \frac{1}{2}|P(x)-Q(x)|$ between the corresponding outcome probability distributions. As expected, in the limit of commuting density matrices, both the fidelity and the trace distance reduce to their classical counterparts, i.e., to the Bhattacharyya overlap and the Kolmogorov distance, respectively.

As is manifested in these examples, density matrices can be thought of as generalizations of classical probability distributions, which include the latter as a special case. However, in many quantum information scenarios, one often deals with an even more general concept, which is a hybrid between the quantum and classical cases. This is the concept of a probabilistic ensemble of quantum states, i.e., a classical probability distribution of density matrices. Ensembles of quantum states describe situations in which a quantum system can take a number of different states at random according to some probability distribution. Such a situation is, for example, the outcome of a quantum measurement. A quantum measurement can be regarded as a stochastic quantum channel that outputs different quantum states with probabilities that depend on the input state according to the generalized Born rule [2]. When the measurement is projective, the possible output states are orthogonal and the output ensemble can be regarded as a classical one. However, in the case of generalized measurements the states need not be orthogonal, and the output of the channel is a genuine quantum ensemble.

A quantum state is said to "...capture the best information available about how a quantum system will react in this or that experimental situation" [13]. Accordingly, a quantum ensemble gives the best information available about how a quantum system will react in this or that experimental situation when the choice of experiment can be made conditional on some classical side information. The uses or applications of the quantum system will depend strongly on the particular quantum states that appear in the ensemble and on their probabilities.

It should be noted that in the context of resource theory [14], a protocol consisting of allowed transformations generally involves measurements, and the resource available after a measurement is given by the average resource of the resulting ensemble. For example, the restriction to local operations and classical communication (LOCC) naturally gives rise to entanglement as a resource, which is quantified by an entanglement monotone - a function which does not increase on average under LOCC [15, 16]. In this sense, entanglement can be thought of as a function defined on ensembles. Ensembles of quantum states have various other applications in quantum information theory, with particularly notable ones in quantum communication, e.g., for representing sources of quantum states used for communication [17, 18], or for describing "static resources" of shared classical-quantum correlations in multi-partite systems [19].
Even though various measures of distance and fidelity between quantum states have been studied, similar measures for ensembles of states have been lacking. With the development of quantum technology, it becomes important to be able to rigorously compare different experimental schemes and assess the degree to which they differ from ideal ones. The existing measures of distance and fidelity between quantum states are sufficient for this purpose when the system of interest at a given stage of the experiment is described by a single quantum state. These measures can also be used to define distance and fidelity between deterministic quantum operations, i.e., completely positive trace-preserving (CPTP) maps [20]. However, in many situations an experiment may involve states obtained randomly according to some probability distribution, such as the states obtained during the process of entanglement concentration [21], or the states resulting from the measurement of an error syndrome during and error-correction protocol [22], or simply a source of quantum states used for communication. It is therefore important to have a distinguishability measure between two ensembles of states. Furthermore, the tools of quantum information involve not only CPTP maps but also stochastic quantum operations (generalized measurements), and a figure of merit comparing two such operations (e.g., a real one with an ideal one) would require a quantitative comparison between their output ensembles. Rigorous measures that compare generalized measurements would be useful, in particular, for assessing the performance of quantum detectors, which can now be characterized experimentally [26] through quantum detector tomography [23, 24, 25].

The purpose of this paper is to propose measures of distance and fidelity between ensembles of quantum states and use them to define distance and fidelity between generalized measurements. The rest of the paper is organized as follows. In Sec. II, we review the concept of an ensemble of quantum states and establish nomenclature. In Sec. III, we discuss some basic properties that we expect a measure of distinguishability between ensembles to have, and rule out several naive candidates. In Sec. IV, we propose measures of distance and fidelity of a Kantorovich type and study their properties. We first introduce the measure of distance on the basis of intuitive considerations concerning the ability of states obtained randomly from one ensemble to mimic states obtained randomly from the other ensemble. The measure is based on the trace distance between states and satisfies a number of desirable properties. In addition to the standard distance properties, it is jointly convex, monotonic under averaging of the ensembles and under CPTP maps. When the ensembles are discrete, the measure is equivalent to a linear program and can be computed efficiently in the size of the set of states participating in the ensembles. We show that for simple limiting cases, the distance between ensembles reduces to intuitive expressions involving the trace distance between states. We introduce a measure of fidelity between ensembles in a similar fashion. The 
fidelity satisfies properties analogous to those of the distance and also can be computed as a linear program. We provide operational interpretations of both quantities. We show that for the case when the measures are based on the trace distance and the standard fidelity, the measures are not monotonic under generalized measurements. We explain why this is natural considering the operational interpretations of the quantities and derive necessary and sufficient conditions which the basic measures of distance or fidelity between states have to satisfy in order for the corresponding Kantorovich measures to be monotonic under measurements. In Sec. V, we propose measures of distance and fidelity which make use of the extended-Hilbert-space (EHS) representation of ensembles [19]. We argue that to every ensemble of quantum states there is a corresponding class of valid EHS representations and provide a rigorous definition of this class. We then define the measures as a minimum (maximum) of the distance (fidelity) between all possible EHS representations of the ensembles being compared. We show that these definitions can be simplified and are equivalent to convex optimization problems. We also provide equivalent formulations without reference to an extended Hilbert space. These quantities are based on the trace distance and the square root fidelity and inherit all their celebrated properties such as joint convexity in the case of the trace distance or strong concavity in the case of the fidelity. In addition, they are monotonic under averaging of the ensembles, as well as under generalized measurements. The latter property can be regarded as a generalization of the monotonicity under CPTP maps of the trace distance and the square root fidelity. The EHS measures are upper (lower) bounded by the Kantorovich distance (fidelity). We provide operational interpretations for the EHS measures too. In Sec. VI, we present a novel interpretation of the standard fidelity between mixed states as a maximum of the fidelity between all pure-state ensembles from which the mixed states being compared can be constructed. The fidelity between pure-state ensembles used in this definition is of the EHS type but can be expressed without any reference to fidelity between mixed states and has a form which can be regarded as a generalization of the Bhattacharyya overlap. In Sec. VII, we use the measures between ensembles of quantum states to define distance and fidelity between generalized measurements. We consider two definitionsone based on the Jamiołkowski isomorphism [27] and another based on worst-case comparison - and discuss their properties. We also propose distance and fidelity between positive operator-valued measures (POVMs). In Sec. VIII, we conclude.

\section{ENSEMBLES OF QUANTUM STATES}

Let $\mathcal{B}(\mathcal{H})$ denote the set of linear operators on a finitedimensional Hilbert space $\mathcal{H}$. For the purposes of this paper, a (probabilistic) ensemble of quantum states is a set of pairs $\left\{\left(p_{x}, \rho_{x}\right)\right\}$ of probabilities $p_{x}\left(p_{x} \geq 0\right.$, $\left.\sum_{x} p_{x}=1\right)$ and distinct density matrices $\rho_{x} \in \overline{\mathcal{B}}(\mathcal{H})$ $\left(\rho_{x}>0, \operatorname{Tr}\left(\rho_{x}\right)=1\right), \rho_{x} \neq \rho_{y}$ for $x \neq y$. For simplicity, we will assume that the set of states participating in an ensemble is discrete (i.e., the index $x$ runs over a countable set), although we expect that our considerations extend to non-discrete ensembles as well. We will use the concept of ensemble of states to describe situations in which a system takes a state $\rho_{x}$ at random with probability $p_{x}$. The statement that a system takes the state $\rho_{x}$ means that there exists classical information about the identity of the state. This is to be distinguished from the situation in which no information about the identity of the state exists or can be obtained. In the latter case, for all practical purposes, the average density matrix of the ensemble, $\bar{\rho}=\sum_{x} p_{x} \rho_{x}$, provides a complete description of the state of the system.

An example of an ensemble of states is the output of a non-destructive generalized measurement. Under the most general type of quantum measurement, a density matrix $\rho$ transforms as

$$
\rho \rightarrow \rho_{i}=\frac{\mathcal{M}_{i}(\rho)}{\operatorname{Tr} \mathcal{M}_{i}(\rho)}, \text { with probability } p_{i}=\operatorname{Tr} \mathcal{M}_{i}(\rho),
$$

where $\mathcal{M}_{i}(\cdot)=\sum_{j} M_{i j}(\cdot) M_{i j}^{\dagger}$ is the measurement superoperator corresponding to measurement outcome $i$. (The operators $M_{i j}$ satisfy the completeness relation $\sum_{i, j} M_{i j}^{\dagger} M_{i j}=I$.) Note that different measurement outcomes do not necessarily yield different output states. For example, both outcomes of a measurement on a qubit system with measurement superoperators $\mathcal{M}_{1}(\cdot)=$ $|0\rangle\langle 0|(\cdot)| 0\rangle\langle 0|$ and $\mathcal{M}_{2}(\cdot)=|0\rangle\langle 1|(\cdot)| 1\rangle\langle 0|$ leave the system in the state $|0\rangle\langle 0|$, although they provide information about the input state. If $\left\{\rho_{x}\right\}$ is the set of distinct output states, each occurring with probability $p_{x}=\sum_{i: \rho_{i}=\rho_{x}} p_{i}$, the ensemble of post-measurement states resulting from the stochastic transformation (3) is $\left\{\left(p_{x}, \rho_{x}\right)\right\}$.

Let $\left\{\left(p_{x}, \rho_{x}\right)\right\}$ be an ensemble of density matrices over a Hilbert space $\mathcal{H}$. If $\Omega_{1}$ is the set of all density matrices $\rho_{x}$ that participate in the ensemble, we can equivalently represent the ensemble as a probability distribution $P(\rho)$, $\rho \in \Omega_{1}\left(P\left(\rho_{x}\right) \equiv p_{x}\right)$, over the set $\Omega_{1}$. Consider a second ensemble, $Q(\sigma), \sigma \in \Omega_{2}$, where the set $\Omega_{2}$ is not necessarily equal to $\Omega_{1}$. We can think of the two ensembles as corresponding to probability distributions over the same set, by extending the definitions of $P(\rho)$ and $Q(\sigma)$ to the larger set $\Omega=\Omega_{1} \cup \Omega_{2}$ through assigning zero probabilities to those states that do not participate in the respective ensembles. Therefore, without loss of generality, we will treat the ensembles that we compare as probability distributions $P(\rho)$ and $Q(\rho)$ over the same set $\Omega$. (Sometimes, when it is clear from the context, we will denote the ensembles we compare simply by $P$ and Q.) Most generally, the set $\Omega$ can be taken to be the full set of density matrices over $\mathcal{H}$, but in this paper we will assume that $\Omega$ is discrete.

The fact that $P(\rho)$ and $Q(\rho)$ are valid probability dis- 
tributions is expressed in the conditions

$$
\begin{aligned}
& \sum_{\rho^{\prime} \in \Omega} P\left(\rho^{\prime}\right)=1, \quad P(\rho) \geq 0, \quad \forall \rho \in \Omega, \\
& \sum_{\rho^{\prime} \in \Omega} Q\left(\rho^{\prime}\right)=1, \quad Q(\rho) \geq 0, \quad \forall \rho \in \Omega .
\end{aligned}
$$

If our world is ultimately quantum, it is natural to expect that an ensemble of quantum states must have a description in terms of the state of a (possibly larger) quantum system. Indeed, there is a correspondence between an ensemble of the form $\left\{\left(p_{x}, \rho_{x}\right)\right\}$ and a state of the form

$$
\widehat{\rho}=\sum_{x} p_{x} \rho_{x} \otimes|x\rangle\langle x|,
$$

where the pointer (or flag) states $\{|x\rangle\}$ are an orthonormal set in the Hilbert space of an auxiliary system of a sufficiently large dimension [19]. The pointer states can be thought of as carrying the classical information about which particular state from the ensemble we are givena measurement of the classical system yields the quantum state $\rho_{x}$ with probability $p_{x}$, which is equivalent to drawing a state randomly from the ensemble. Reversely, if we are given a state drawn randomly from the ensemble, we can record our knowledge about the identity of the state in a 'classical' pointer attached to it and forget the information about the state since this information is stored in the pointer and can always be retrieved. After the latter operation, the state of the original system plus the pointer system is described by $\sum_{x} p_{x} \rho_{x} \otimes|x\rangle\langle x|$. This representation is referred to as an extended-Hilbert-space (EHS) representation of an ensemble [19]. For simplicity and in order to distinguish the system storing the classical memory from the quantum system, we will use the following notation for the pointers:

$$
[x] \equiv|x\rangle\langle x| .
$$

In this notation, the state (6) reads

$$
\widehat{\rho}=\sum_{x} p_{x} \rho_{x} \otimes[x] .
$$

In terms of the description of an ensemble as a probability distribution $P(\rho)$ over a set of states $\Omega$, an EHS representation of this type can be written as

$$
\widehat{\rho}_{P}=\sum_{\rho \in \Omega} P(\rho) \rho \otimes[\rho],
$$

where $\{[\rho]\}$ is an orthonormal set of pure pointer states $|\rho\rangle\langle\rho|$, each of which is associated with a unique density matrix $\rho \in \Omega$. We will develop this concept further in Sec. V.

\section{NAIVE CANDIDATES}

Before we propose distinguishability measures between two ensembles of quantum states, let us first consider what properties we expect such measures to have. The answer to this question will depend on the operational context in which we want to compare the ensembles.

We could ask, for example, how different on average two states drawn randomly from the two ensembles are. Given a measure of distance $d(\rho, \sigma)$ between states, the average distance in that sense would be $\sum \sum P(\rho) Q(\sigma) d(\rho, \sigma)$. This quantity obviously could $\rho \in \Omega \sigma \in \Omega$

be non-zero even when the two ensembles are identical. Similarly, we could look at the average fidelity which can be smaller than 1 for identical ensembles. Thus even though these quantities have a well defined meaning, they are not good measures of distinguishability.

Another possibility is to look at a distance $d\left(\bar{\rho}_{P}, \bar{\rho}_{Q}\right)$ between the average density matrices $\bar{\rho}_{P}=\sum_{\rho \in \Omega} P(\rho) \rho$ and $\bar{\rho}_{Q}=\sum_{\rho \in \Omega} Q(\rho) \rho$ of the two ensembles, or the fidelity $F\left(\bar{\rho}_{P}, \bar{\rho}_{Q}\right)$ between them. Obviously, for identical ensembles the distance is equal to 0 and the fidelity is equal to 1 . However, these quantities cannot discriminate between different ensembles that have the same average density matrices. Imagine, for example, that an experimentalist has at her disposal two devices. The first one produces the two-qubit Bell states $\frac{|00\rangle+|11\rangle}{\sqrt{2}}, \frac{|00\rangle-|11\rangle}{\sqrt{2}}$, $\frac{|01\rangle+|10\rangle}{\sqrt{2}}, \frac{|01\rangle-|10\rangle}{\sqrt{2}}$, each occurring with probability $1 / 4$, together with a classical indicator specifying which state is produced. The second device produces the two-qubit product states $|00\rangle,|01\rangle,|10\rangle,|11\rangle$, each occurring with probability $1 / 4$, again with an indicator of the identity of the state. Although the average states in the two cases are the same, the ensembles produced by the two devices have very different properties. In the first case, the average entanglement between the two qubits is maximal, whereas in the second case it is zero. Therefore, in order to capture the difference between two ensembles, we would like our measure of distance (fidelity) to be 0 (1) if and only if $P(\rho)=Q(\rho), \forall \rho \in \Omega$.

Measures of distance and fidelity which satisfy the latter requirement could be any measures of distance and fidelity between probability distributions which treat $\rho$ as a classical variable. Consider, for example, the Kolmogorov distance $\frac{1}{2} \sum_{\rho \in \Omega}|P(\rho)-Q(\rho)|$. Note that this distance is precisely equal to the trace distance between the EHS representations of the two ensembles of type (9),

$$
\begin{gathered}
\Delta\left(\widehat{\rho}_{P}, \widehat{\rho}_{Q}\right)=\frac{1}{2}\left\|\sum_{\rho \in \Omega} P(\rho) \rho \otimes[\rho]-\sum_{\sigma \in \Omega} Q(\sigma) \sigma \otimes[\sigma]\right\| \\
=\frac{1}{2} \sum_{\rho \in \Omega}|P(\rho)-Q(\rho)| .
\end{gathered}
$$

In a similar manner, we could look at the Bhattacharyya overlap $\sum_{\rho \in \Omega} \sqrt{P(\rho) Q(\rho)}$, which is equal to the fidelity between the two EHS representations of type (9). Such measures, however, do not take into account the quantum-mechanical aspect of the variables $\rho$. If the two 
distributions $P$ and $Q$ have supports on non-overlapping subsets of $\Omega$, the above distance (fidelity) would be maximal (minimal), but as we mentioned earlier, two distinct density matrices are not necessarily distinguishable (they often behave as if they are the same state) and we would like our distance and fidelity to capture this property. In particular, in the special case where each of the two ensembles consists of a single state, we would like the measures between the two ensembles to be equal to the distance or fidelity between the respective states. If we used the above distance (fidelity) between classical probability distributions in this case, we would obtain a maximum (minimum) value even if the two states are very similar. At the same time, it is natural to expect that a distance between ensembles would reduce to a distance between classical probability distributions when the states participating in the ensembles are orthogonal.

\section{DISTANCE AND FIDELITY OF A KANTOROVICH TYPE}

\section{A. Motivating the definitions}

The above examples suggest that distinguishability measures with the desired properties may have to be nontrivial functions of the probability distributions and the set of states participating in the ensembles. Heuristically, a distance (fidelity) between two quantum states can be regarded as a measure of the extent to which the two states do not (do) behave as if they are the same state (the precise meaning of this statement depends on the operational meaning of the distance (fidelity) in question). In a similar manner, we would expect a distance (fidelity) between two ensembles of quantum states to compare the extent to which the two ensembles do not (do) "behave" as if they are the same ensemble. Since the ensemble is a statistical concept which describes the situation of having particular states with particular probabilities, we would like to compare the extent to which states drawn randomly from one ensemble can be used to mimic states drawn randomly from the other ensemble.

When states drawn randomly from the ensemble $\{(Q(\sigma), \sigma)\}$ are used to mimic states drawn from the ensemble $\{(P(\rho), \rho)\}$, a given state $\sigma$ obtained according to the distribution $Q(\sigma)$ most generally can be taken with different probabilities to pass off as different states $\rho$ from $\{(P(\rho), \rho)\}$. In other words, the process of mimicking one ensemble using the other one as a resource can be described by a transition probability matrix whose elements $T(\rho \mid \sigma), \rho, \sigma \in \Omega$, describe the probabilities with which the state $\sigma$ sampled from the distribution $Q(\sigma)$ is taken to pass off as the state $\rho$ sampled from $P(\rho)$. The requirement that under this simulation the probabilities are consistent with the probabilities $P(\rho)$ and $Q(\sigma)$, respectively, is expressed in the condition $\sum_{\sigma \in \Omega} T(\rho \mid \sigma) Q(\sigma)=P(\rho)$. The fact that $T(\rho \mid \sigma)$ describe valid transition probabil- ities imposes the conditions $T(\rho \mid \sigma) \geq 0, \forall \rho, \sigma \in \Omega$, and $\sum_{\rho \in \Omega} T(\rho \mid \sigma)=1, \forall \sigma \in \Omega$.

In order to measure how much the state $\sigma$ fails to mimic the state $\rho$, we can use any measure of distance between states. In this paper, we will concentrate on the case of the trace distance, $\Delta(\rho, \sigma)(\mathrm{Eq}$. (2) $)$. To measure the degree to which a map $T(\rho \mid \sigma)$ from one ensemble to the other fails to mimic the latter, we propose to use the average distance between the actual states and those that they mimic: $\sum_{\rho, \sigma \in \Omega} T(\rho \mid \sigma) Q(\sigma) \Delta(\rho, \sigma)$. We can write this expression in an explicitly symmetric form by introducing the joint probability distribution $\Pi(\rho, \sigma) \equiv T(\rho \mid \sigma) Q(\sigma)$ which satisfies the marginal conditions $\sum_{\sigma \in \Omega} \Pi(\rho, \sigma)=P(\rho), \quad \forall \rho \in \Omega$, and $\sum_{\rho \in \Omega} \Pi(\rho, \sigma)=Q(\sigma), \quad \forall \sigma \in \Omega$ :

$$
D_{\Pi}(P, Q)=\sum_{\rho, \sigma \in \Omega} \Pi(\rho, \sigma) \Delta(\rho, \sigma) .
$$

Clearly, different choices of the map $T(\rho \mid \sigma)$ (or equivalently, of $\Pi(\rho, \sigma))$ can yield different values for the quantity (11). Therefore, we define the distance between the two ensembles as the minimum of the quantity (11) over all possible choices of $\Pi(\rho, \sigma)$, i.e., we choose the optimal mimicking strategy.

Definition 1 (Kantorovich distance). Let $P(\rho)$ and $Q(\rho), \rho \in \Omega$, be two ensembles (probability distributions over $\Omega$ ), which we denote by $P$ and $Q$ for short. Then

$$
D^{K}(P, Q)=\min _{\Pi(\rho, \sigma)} \sum_{\rho, \sigma \in \Omega} \Pi(\rho, \sigma) \Delta(\rho, \sigma),
$$

where minimum is taken over all joint probability distributions $\Pi(\rho, \sigma)$ with marginals $\sum_{\sigma \in \Omega} \Pi(\rho, \sigma)=$ $P(\rho), \quad \forall \rho \in \Omega$, and $\sum_{\rho \in \Omega} \Pi(\rho, \sigma)=Q(\sigma), \forall \sigma \in \Omega$.

The quantity (12) is of the same form as the Kantorovich formulation of the optimal transportation problem [28], which is a relaxation of a problem studied in 1781 by Monge. In 1975, Kantorovich received the Nobel Prize in Economics, together with Koopmans, for their contributions to the theory of optimum allocation of resources, and he is considered to be one of the fathers of linear programming. The optimal transportation problem can be cast in the spirit of its original formulations as follows:

Assume you have to transport the coal produced in some mines $X$ to the factories $Y$. The amounts produced in each mine $\left\{P_{1}, P_{2}, \ldots\right\}$ as well as the needs for each factory $\left\{Q_{1}, Q_{2}, \ldots\right\}$ are given. There is a cost per unit of mass $c(x, y)$ to move coal from mine $x$ to factory $y$. The problem is to find the optimal transportation plan or transportation map $T(y \mid x)$, i.e., for every mine $x$ determine how much material has to be carried to every factory y so as to minimize the overall cost.

The analogy with the above definition (12) is straightforward: mines and factories play the role of the quantum states $\rho$ and $\sigma$ in each ensemble respectively, and the cost 
function is given by the trace distance. Kantorovich's formulation extended also to non-discrete probability measures 29] and was one of the first infinite-dimensional linear programming problems to be considered. If the probability measures are defined over a metric space and the cost function is taken to be the corresponding distance function, the optimal average cost is known as the Kantorovich distance (also referred to as Wasserstein distance [30]). The optimal transportation problem is now an active field of research with tight connections with problems in geometry, probability theory, differential equations, fluid mechanics, economics and image or data processing.

Based on the same idea we can define a fidelity between two ensembles, which we will refer to as the Kantorovich fidelity.

Definition 2 (Kantorovich fidelity). The Kantorovich fidelity between the ensembles $P(\rho)$ and $Q(\rho)$, $\rho \in \Omega$, is

$$
F^{K}(P, Q)=\max _{\Pi(\rho, \sigma)} \sum_{\rho, \sigma \in \Omega} \Pi(\rho, \sigma) F(\rho, \sigma),
$$

where $F(\rho, \sigma)$ is the square root fidelity between $\rho$ and $\sigma$ (Eq. (1) ), and maximum is taken over all joint probability distributions $\Pi(\rho, \sigma)$ that satisfy $\sum_{\sigma \in \Omega} \Pi(\rho, \sigma)=$ $P(\rho), \quad \forall \rho \in \Omega$, and $\sum_{\rho \in \Omega} \Pi(\rho, \sigma)=Q(\sigma), \quad \forall \sigma \in \Omega$.

\section{B. Properties of the Kantorovich distance}

Let $\mathcal{P}_{\Omega}$ denote the set of probability distributions over a set of density matrices $\Omega$.

Property 1 (Positivity).

$$
\begin{gathered}
D^{K}(P, Q) \geq 0, \\
\forall P, Q \in \mathcal{P}_{\Omega},
\end{gathered}
$$

with equality

$$
D^{K}(P, Q)=0 \text { iff } P(\rho)=Q(\rho), \forall \rho \in \Omega .
$$

Proof. Since all terms in Eq. (12) are non-negative, the distance $D^{K}(P, Q)$ is also non-negative. Obviously, if $P(\rho)=Q(\rho), \forall \rho \in \Omega$, we obtain $D^{K}(P, Q)=0$ by choosing the joint probability distribution $\Pi(\rho, \sigma)=\delta_{\rho, \sigma} P(\rho)$. Inversely, assume that $D^{K}(P, Q)=0$. This means that all terms in Eq. (12) must be zero, which can happen only if $\Pi(\rho, \sigma) \propto \delta_{\rho, \sigma}$. From the condition for the marginal probability distributions, we see that $\Pi(\rho, \sigma)=\delta_{\rho, \sigma} P(\rho)$ and $P(\rho)=Q(\rho)$.

Property 2 (Normalization).

$$
\begin{aligned}
& D^{K}(P, Q) \leq 1, \\
& \forall P, Q \in \mathcal{P}_{\Omega},
\end{aligned}
$$

with equality

$$
D^{K}(P, Q)=1
$$

if and only if the supports of $\mathrm{P}$ and $\mathrm{Q}$ are orthogonal sets of states.

Proof. Since $\Delta(\rho, \sigma) \leq 1$, then for any given $\Pi(\rho, \sigma)$ we have $\sum_{\rho, \sigma \in \Omega} \Pi(\rho, \sigma) \bar{\Delta}(\rho, \sigma) \leq \sum_{\rho, \sigma \in \Omega} \Pi(\rho, \sigma)=1$. Furthermore, $\Delta(\rho, \sigma)=1$ if and only if $\rho$ and $\sigma$ are orthogonal. Observe that the only non-zero values $\Pi(\rho, \sigma)$ of a joint probability distribution that respects the marginal distributions $P$ and $Q$ are those for which $\rho$ is in the support of $P$ and $\sigma$ is in the support of $Q$. Therefore, if $P$ and $Q$ have supports on sets of density matrices which are orthogonal, every non-zero component $\Pi(\rho, \sigma)$ in the sum on the right-hand side of Eq. (12) will be multiplied by $\Delta(\rho, \sigma)=1$, which implies that $D^{K}(P, Q)=1$. Inversely, since $\sum_{\rho, \sigma \in \Omega} \Pi(\rho, \sigma)=1$ if $D^{K}(P, Q)=1$, then every non-zero $\Pi(\rho, \sigma)$ on the righthand side of Eq. (12) must be multiplied by 1, which implies that $P$ and $Q$ must have supports on orthogonal sets.

Property 3 (Symmetry).

$$
\begin{gathered}
D^{K}(P, Q)=D^{K}(Q, P), \\
\forall P, Q \in \mathcal{P}_{\Omega} .
\end{gathered}
$$

Proof. The symmetry follows from the definition (12) and the symmetry of $\Delta(\rho, \sigma)$.

\section{Property 4 (Triangle inequality).}

$$
\begin{aligned}
D^{K}(P, R) & \leq D^{K}(P, Q)+D^{K}(Q, R), \\
& \forall P, Q, R \in \mathcal{P}_{\Omega} .
\end{aligned}
$$

Proof. Let $\Pi^{P Q}(\rho, \sigma)$ and $\Pi^{Q R}(\rho, \sigma)$ be the two joint probability distributions which achieve the minimum in Eq. (12) for the pairs of distributions $(P, Q)$ and $(Q, R)$, respectively. Consider the quantity

$$
\tilde{\Pi}^{P R}(\rho, \sigma)=\sum_{\kappa \in \Omega} \Pi^{P Q}(\rho, \kappa) \frac{1}{Q(\kappa)} \Pi^{Q R}(\kappa, \sigma), \quad \rho, \sigma \in \Omega
$$

where for $Q(\kappa)=0$, we define $\Pi^{P Q}(\rho, \kappa) \frac{1}{Q(\kappa)} \Pi^{Q R}(\kappa, \sigma)=0$ (note that if $Q(\kappa)=0$, then $\left.\Pi^{P Q}(\rho, \kappa)=\Pi^{Q R}(\kappa, \sigma)=0, \forall \rho, \sigma \in \Omega\right)$. One can readily verify that this is a valid joint probability distribution with marginals $P$ and $R$. Therefore, we have

$$
\begin{gathered}
D^{K}(P, R) \leq \sum_{\rho, \sigma \in \Omega} \tilde{\Pi}^{P R}(\rho, \sigma) \Delta(\rho, \sigma) \\
=\sum_{\rho, \sigma, \kappa \in \Omega} \Pi^{P Q}(\rho, \kappa) \frac{1}{Q(\kappa)} \Pi^{Q R}(\kappa, \sigma) \Delta(\rho, \sigma) \\
\leq \sum_{\rho, \sigma, \kappa \in \Omega} \Pi^{P Q}(\rho, \kappa) \frac{1}{Q(\kappa)} \Pi^{Q R}(\kappa, \sigma) \Delta(\rho, \kappa) \\
+\sum_{\rho, \sigma, \kappa \in \Omega} \Pi^{P Q}(\rho, \kappa) \frac{1}{Q(\kappa)} \Pi^{Q R}(\kappa, \sigma) \Delta(\kappa, \sigma) \\
=\sum_{\rho, \kappa \in \Omega} \Pi^{P Q}(\rho, \kappa) \Delta(\rho, \kappa)+\sum_{\sigma, \kappa \in \Omega} \Pi^{Q R}(\kappa, \sigma) \Delta(\kappa, \sigma) \\
=D^{K}(P, Q)+D^{K}(Q, R),
\end{gathered}
$$


where in the second inequality we have used the triangle inequality for $\Delta$.

Property 5 (Joint convexity).

$$
\begin{aligned}
& D^{K}\left(p P_{1}+(1-p) P_{2}, p Q_{1}+(1-p) Q_{2}\right) \\
& \leq p D^{K}\left(P_{1}, Q_{1}\right)+(1-p) D^{K}\left(P_{2}, Q_{2}\right), \\
& \quad \forall P_{1}, P_{2}, Q_{1}, Q_{2} \in \mathcal{P}_{\Omega}, \quad \forall p \in[0,1] .
\end{aligned}
$$

Proof. Let $\Pi^{1}(\rho, \sigma)$ and $\Pi^{2}(\rho, \sigma)$ be two joint probability distributions which achieve the minimum in Eq. (12) for the pairs of distributions $\left(P_{1}, Q_{1}\right)$ and $\left(P_{2}, Q_{2}\right)$, respectively. It is immediately seen that

$$
\tilde{\Pi}^{12}(\rho, \sigma)=p \Pi^{1}(\rho, \sigma)+(1-p) \Pi^{2}(\rho, \sigma)
$$

is a joint probability distribution with marginals $p P_{1}+$ $(1-p) P_{2}$ and $p Q_{1}+(1-p) Q_{2}$. Therefore,

$$
\begin{gathered}
D^{K}\left(p P_{1}+(1-p) P_{2}, p Q_{1}+(1-p) Q_{2}\right) \\
\leq \sum_{\rho, \sigma \in \Omega} \tilde{\Pi}^{12}(\rho, \sigma) \Delta(\rho, \sigma) \\
=p \sum_{\rho, \sigma \in \Omega} \Pi^{1}(\rho, \sigma) \Delta(\rho, \sigma)+(1-p) \sum_{\rho, \sigma \in \Omega} \Pi^{2}(\rho, \sigma) \Delta(\rho, \sigma) \\
=p D^{K}\left(P_{1}, Q_{1}\right)+(1-p) D^{K}\left(P_{2}, Q_{2}\right) .
\end{gathered}
$$

Property 6 (Monotonicity under CPTP maps).

Let $\mathcal{E}: \mathcal{B}(\mathcal{H}) \rightarrow \mathcal{B}\left(\mathcal{H}^{\prime}\right)$, where $\mathcal{H}$ and $\mathcal{H}^{\prime}$ generally can have different dimensions, be a completely positive tracepreserving (CPTP) map. (Any such map can be written in the Kraus form $\mathcal{E}(\rho)=\sum_{i} M_{i} \rho M_{i}^{\dagger}, \forall \rho \in \mathcal{B}(\mathcal{H})$ 31]). Denote the set of density matrices consisting of $\mathcal{E}(\rho)$, with $\rho \in \Omega$, by $\Omega_{\mathcal{E}}$. If we apply the same CPTP map $\mathcal{E}$ to every state in an ensemble $P(\rho), \rho \in \Omega$, we obtain another ensemble $P^{\prime}\left(\rho^{\prime}\right), \rho^{\prime} \in \Omega_{\mathcal{E}}$. Note that generally $P(\rho) \neq P^{\prime}(\mathcal{E}(\rho))$, because the map $\mathcal{E}$ may be such that it takes two or more different states from $\Omega$ to one and the same state in $\Omega_{\mathcal{E}}$, e.g., $\mathcal{E}\left(\rho_{1}\right)=\mathcal{E}\left(\rho_{2}\right), \rho_{1} \in \Omega$, $\rho_{2} \in \Omega, \rho_{1} \neq \rho_{2}$. (The opposite obviously cannot happen because every state $\rho$ in $\Omega$ is mapped to a unique state $\mathcal{E}(\rho) \in \Omega_{\mathcal{E}}$.) Thus the operation $\mathcal{E}$ induces a map from the set of probability distributions over $\Omega$ to the set of probability distributions over $\Omega_{\mathcal{E}}$. Denote this map by $M_{\mathcal{E}}: \mathcal{P}_{\Omega} \rightarrow \mathcal{P}_{\Omega_{\mathcal{E}}}$.

Now we can state the property of monotonicity under CPTP maps as follows: For all CPTP maps $\mathcal{E}$,

$$
D^{K}(P, Q) \geq D^{K}\left(M_{\mathcal{E}}(P), M_{\mathcal{E}}(Q)\right),
$$

where $M_{\mathcal{E}}: \mathcal{P}_{\Omega} \rightarrow \mathcal{P}_{\Omega_{\mathcal{E}}}$ is the map induced by $\mathcal{E}$.

Proof. Let $\Pi(\rho, \sigma)$ be a joint probability distribution for which the minimum in the definition (12) of $D^{K}(P, Q)$ is attained. Observe that $\sum_{\rho, \sigma \in \Omega} \Pi(\rho, \sigma) \Delta(\mathcal{E}(\rho), \mathcal{E}(\sigma))=$ $\sum_{\rho^{\prime}, \sigma^{\prime} \in \Omega_{\mathcal{E}}} \Pi^{\prime}\left(\rho^{\prime}, \sigma^{\prime}\right) \Delta\left(\rho^{\prime}, \sigma^{\prime}\right)$, where $\Pi^{\prime}\left(\rho^{\prime}, \sigma^{\prime}\right)$ is a joint probability distribution over $\Omega_{\mathcal{E}} \times \Omega_{\mathcal{E}}$ with marginals $P^{\prime}\left(\rho^{\prime}\right)$ and $Q\left(\rho^{\prime}\right)$. This can be seen from the fact that $P^{\prime}\left(\rho^{\prime}\right)=\sum_{x} P\left(\rho_{x}\right)$, where the sum is over all $\rho_{x} \in \Omega$ such that $\rho^{\prime}=\mathcal{E}\left(\rho_{x}\right)$. Similarly, $Q^{\prime}\left(\sigma^{\prime}\right)=\sum_{y} P\left(\sigma_{y}\right)$, where the sum is over all $\sigma_{y} \in \Omega$ such that $\sigma^{\prime}=\mathcal{E}\left(\sigma_{y}\right)$. Therefore, we have that

$$
\begin{gathered}
D^{K}\left(M_{\mathcal{E}}(P), M_{\mathcal{E}}(Q)\right) \leq \sum_{\rho^{\prime}, \sigma^{\prime} \in \Omega_{\mathcal{E}}} \Pi^{\prime}\left(\rho^{\prime}, \sigma^{\prime}\right) \Delta\left(\rho^{\prime}, \sigma^{\prime}\right) \\
=\sum_{\rho, \sigma \in \Omega} \Pi(\rho, \sigma) \Delta(\mathcal{E}(\rho), \mathcal{E}(\sigma)) \leq \sum_{\rho, \sigma \in \Omega} \Pi(\rho, \sigma) \Delta(\rho, \sigma) \\
=D^{K}(P, Q),
\end{gathered}
$$

where the last inequality follows from the monotonicity of $\Delta(\rho, \sigma)$ under CPTP maps [32].

Corollary (Invariance under unitary maps).

For all unitary maps $\mathcal{U}$,

$$
D^{K}(P, Q)=D^{K}\left(M_{\mathcal{U}}(P), M_{\mathcal{U}}(Q)\right) .
$$

The property follows from the fact that unitary maps are reversible CPTP maps.

Property 7 (Monotonicity under averaging). Let $\bar{P}$ denote the singleton ensemble consisting of the average state of $P(\rho), \bar{\rho}_{P}=\sum_{\rho \in \Omega} P(\rho) \rho$. Then

$$
D^{K}(P, Q) \geq D^{K}(\bar{P}, \bar{Q}) .
$$

Proof. Let $\Pi(\sigma, \rho)$ be a joint probability distribution for which the minimum in the definition (12) of $D(P, Q)$ is attained. Since $\Delta(\rho, \sigma)$ is jointly convex [2], we have

$$
\begin{gathered}
D^{K}(P, Q)=\sum_{\rho, \sigma \in \Omega} \Pi(\rho, \sigma) \Delta(\rho, \sigma) \\
\geq \Delta\left(\sum_{\rho, \sigma \in \Omega} \Pi(\rho, \sigma) \rho, \sum_{\rho, \sigma \in \Omega} \Pi(\rho, \sigma) \sigma\right) \\
=\Delta\left(\sum_{\rho \in \Omega} P(\rho) \rho, \sum_{\sigma \in \Omega} Q(\sigma) \sigma\right) \\
=\Delta\left(\bar{\rho}_{P}, \bar{\rho}_{Q}\right)=D^{K}(\bar{P}, \bar{Q}) .
\end{gathered}
$$

(For the last equality, see Eq. (48) below.)

Corollary. If two distributions are close, their average states are also close, i.e.,

$$
\text { if } D^{K}(P, Q) \leq \varepsilon \text {, then } \Delta\left(\bar{\rho}_{P}, \bar{\rho}_{Q}\right) \leq D^{K}(P, Q) \leq \varepsilon \text {. }
$$

Property 8 (Continuity of the average of a continuous function). Let $h(\rho)$ be a bounded function, which is continuous with respect to the distance $\Delta$. Then the ensemble average of $h(\rho), \bar{h}_{P}=\sum_{\rho \in \Omega} P(\rho) h(\rho)$, is continuous with respect to $D^{K}$.

Proof. The proof is presented in Appendix A.

Comment. Property 8 naturally reflects the idea of states as resources. Assuming that a resource is a continuous function of the state, if two ensembles are close, their corresponding average resources must also be close.

Example (Continuity of the Holevo information). A function of ensembles, which is of great significance in quantum information theory, is the Holevo 
information [18]

$$
\chi(P)=S(\bar{\rho})-\sum_{x} p_{x} S\left(\rho_{x}\right) .
$$

Here $\bar{\rho}=\sum_{x} p_{x} \rho_{x}$ is the average density matrix of the ensemble $\left\{\left(p_{x}, \rho_{x}\right)\right\}$ which we denote by $P$ for short, and $S(\rho)=-\operatorname{Tr}(\rho \log \rho)$ is the von Neumann entropy. This function gives an upper bound to the amount of information about the index $x$ extractable through measurements on a state obtained randomly from the ensemble and is used to define the classical capacity of a quantum channel under independent uses of the channel [33, 34]. The second term in the expression (31) is the average of the von Neumann entropy over the ensemble, while the first term is the von Neumann entropy of the average. Since $S(\rho)$ is a continuous function, from Property 8 and the Corollary of Property 7 one can easily see that the Holevo information is a continuous function of the ensemble with respect to the Kantorovich distance. It would be interesting, however, to obtain an explicit bound of that continuity. For this purpose, we will need the following lemma.

Lemma 1. If a function $h(\rho)$ satisfies the continuity property

$$
|h(\rho)-h(\sigma)| \leq g[\Delta(\rho, \sigma)]
$$

for some function $g[x]$ that is concave in $x \in[0,1]$, then the ensemble average of $h(\rho)$ satisfies

$$
\left|\bar{h}_{P}-\bar{h}_{Q}\right| \leq g\left[D^{K}(P, Q)\right] .
$$

Proof. Let $\Pi(\rho, \sigma)$ be a joint probability distribution which attains the minimum in Eq. (12) for the distributions $P$ and $Q$. Then,

$$
\begin{gathered}
\left|\bar{h}_{P}-\bar{h}_{Q}\right|=\left|\sum_{\rho \in \Omega} P(\rho) h(\rho)-\sum_{\sigma \in \Omega} Q(\sigma) h(\sigma)\right| \\
=\left|\sum_{\rho, \sigma \in \Omega} \Pi(\rho, \sigma) h(\rho)-\sum_{\rho, \sigma \in \Omega} \Pi(\rho, \sigma) h(\sigma)\right| \\
\leq \sum_{\rho, \sigma \in \Omega} \Pi(\rho, \sigma)|h(\rho)-h(\sigma)| \leq \sum_{\rho, \sigma \in \Omega} \Pi(\rho, \sigma) g[\Delta(\rho, \sigma)] \\
\leq g\left[\sum_{\rho, \sigma \in \Omega} \Pi(\rho, \sigma) \Delta(\rho, \sigma)\right]=g\left[D^{K}(P, Q)\right] .
\end{gathered}
$$

Theorem 1 (A Fannes-type inequality for the ensemble average of the von Neumann entropy). For any two ensembles $P$ and $Q$ of density matrices over a $d$-dimensional Hilbert space,

$$
\left|\bar{S}_{P}-\bar{S}_{Q}\right| \leq D^{K} \log _{2}(d-1)+H\left(\left(D^{K}, 1-D^{K}\right)\right),
$$

where $D^{K}$ is the Kantorovich distance between the ensembles $P$ and $Q$, and $H\left(\left(D^{K}, 1-D^{K}\right)\right)=$ $-D^{K} \log _{2}\left(D^{K}\right)-\left(1-D^{K}\right) \log _{2}\left(1-D^{K}\right)$ is the Shannon entropy of the binary probability distribution $\left(D^{K}, 1-\right.$ $\left.D^{K}\right)$.
Comment. This inequality is based on a Fannes-type inequality for the von Neumann entropy due to Audenaert [35], which is stronger than the original inequality by Fannes [36] and provides the sharpest continuity bound for the von Neumann entropy based on $\Delta$ and $d$.

Proof. In Ref. [35], it was shown that

$$
|S(\rho)-S(\sigma)| \leq \Delta \log _{2}(d-1)+H((\Delta, 1-\Delta)) .
$$

The theorem follows from Lemma 1 and the fact that the right-hand side of Eq. (36) is a concave function of $\Delta$.

Corollary (Continuity bound for the Holevo information). The term $S(\bar{\rho})$ in the expression (31) for the Holevo information is not an average of a function, but according to the Corollary of Property 7, $\Delta(\bar{\sigma}, \bar{\rho}) \leq D^{K}(P, Q)$. The right-hand side of Eq. (36) is monotonically increasing in the interval $0 \leq \Delta \leq$ $(d-1) / d$ and monotonically decreasing in the interval $(d-1) / d<\Delta \leq 1$. Therefore, we can write

$$
\begin{gathered}
|S(\bar{\sigma})-S(\bar{\rho})| \leq D^{K} \log _{2}(d-1)+H\left(\left(D^{K}, 1-D^{K}\right)\right) \\
\text { for } 0 \leq D^{K} \leq(d-1) / d
\end{gathered}
$$

Combining Eq. (35) and Eq. (37), we obtain

$$
\begin{gathered}
|\chi(Q)-\chi(P)| \leq 2 D^{K} \log _{2}(d-1)+2 H\left(\left(D^{K}, 1-D^{K}\right)\right) \\
\text { for } 0 \leq D^{K} \leq(d-1) / d .
\end{gathered}
$$

For the interval $(d-1) / d<D^{K} \leq 1$, we can upper bound $|S(\bar{\sigma})-S(\bar{\rho})|$ by its maximum value $\log _{2}(d)$, and we can write the weaker inequality

$$
\begin{gathered}
|\chi(Q)-\chi(P)| \leq \\
\log _{2}(d)+D^{K} \log _{2}(d-1)+H\left(\left(D^{K}, 1-D^{K}\right)\right) \\
\text { for }(d-1) / d<D^{K} \leq 1 .
\end{gathered}
$$

Property 9 (Stability). Let $P(\rho), \rho \in \Omega$, and $R\left(\sigma^{\prime}\right)$, $\sigma^{\prime} \in \Omega^{\prime}$, be two ensembles of quantum states, where $\Omega$ and $\Omega^{\prime}$ are sets of states of two different systems. Define the tensor product of the two ensembles as the ensemble $\left\{\left(P(\rho) R\left(\sigma^{\prime}\right), \rho \otimes \sigma^{\prime}\right)\right\}$, which we will denote by $P \otimes R$ for short. Let $P(\rho)$ and $Q(\rho)$ be two ensembles of states in $\Omega$ and $R\left(\sigma^{\prime}\right)$ be an ensemble of states in $\Omega^{\prime}$. Then,

$$
D^{K}(P \otimes R, Q \otimes R)=D^{K}(P, Q) .
$$

Comment. The physical meaning of this property is that unrelated ensembles do not affect the value of $D^{K}(P, Q)$. Even though this may seem as a natural property to expect from a distance, it does not hold in general even for distance measures between states. For example, the Hilbert-Schmidt distance $\sqrt{\operatorname{Tr}(\rho-\sigma)^{2}}$, which has a well-defined operational meaning [7], is not stable.

Proof. Let

$$
\begin{gathered}
D^{K}(P \otimes R, Q \otimes R)= \\
\sum_{\rho, \sigma \in \Omega ; \tau^{\prime}, \kappa^{\prime} \in \Omega^{\prime}} \Pi\left(\rho \otimes \tau^{\prime}, \sigma \otimes \kappa^{\prime}\right) \Delta\left(\rho \otimes \tau^{\prime}, \sigma \otimes \kappa^{\prime}\right),
\end{gathered}
$$


where $\Pi\left(\rho \otimes \tau^{\prime}, \sigma \otimes \kappa^{\prime}\right)$ has left and right marginals $P(\rho) R\left(\tau^{\prime}\right)$ and $Q(\sigma) R\left(\kappa^{\prime}\right)$, respectively. From the monotonicity of $\Delta$ under partial tracing it follows that

$$
D^{K}(P \otimes R, Q \otimes R) \geq \sum_{\rho, \sigma \in \Omega} \Pi^{\prime}(\rho, \sigma) \Delta(\rho, \sigma),
$$

where

$$
\Pi^{\prime}(\rho, \sigma)=\sum_{\tau^{\prime}, \kappa^{\prime} \in \Omega^{\prime}} \Pi\left(\rho \otimes \tau^{\prime}, \sigma \otimes \kappa^{\prime}\right)
$$

is a joint probability distribution with left and right marginals $P(\rho)$ and $Q(\sigma)$, respectively. Therefore,

$$
D^{K}(P \otimes R, Q \otimes R) \geq D^{K}(P, Q) .
$$

But by choosing $\Pi\left(\rho \otimes \tau^{\prime}, \sigma \otimes \kappa^{\prime}\right)=\Pi(\rho, \sigma) R\left(\tau^{\prime}\right) \delta_{\tau^{\prime} \kappa^{\prime}}$, where $\Pi(\rho, \sigma)$ is a joint distribution which attains the minimum in the definition (12) of $D^{K}(P, Q)$, and using the stability of $\Delta$, the equality in Eq. (44) is attained. This completes the proof.

Property 10 (Linear programming). The task of finding the optimal $\Pi(\sigma, \rho)$ in Eq.(12) is a linear program and can be solved efficiently in the cardinality of $\Omega$.

Proof. If the cardinality of $\Omega$ is $\mathrm{N}$, we can think of $\Delta(\rho, \sigma), \rho, \sigma \in \Omega$ as the components $c_{\mu}, \mu=(\rho, \sigma)$, of an $N^{2}$-component vector which we will denote by $c$. The joint probability distribution $\Pi(\rho, \sigma)$ over which we want to minimize the expression on the right-hand side of Eq. (12) can similarly be thought of as an $N^{2}$-component vector $x$ with components $x_{\mu}, \mu=(\rho, \sigma)$. Thus the task of finding the optimal $\Pi(\rho, \sigma)$ can be expressed in the compact form

$$
\text { Minimize } c^{T} x .
$$

The constraints $\sum_{\sigma \in \Omega} \Pi(\rho, \sigma)=P(\rho), \forall \rho \in \Omega$, and $\sum_{\rho \in \Omega} \Pi(\rho, \sigma)=Q(\sigma), \forall \sigma \in \Omega$, can also be expressed in a compact matrix forms as

$$
\begin{aligned}
& A x=a, \\
& B x=b,
\end{aligned}
$$

where $A$ is an $N \times N^{2}$ matrix with components $A_{\kappa \mu}=\delta_{\kappa \rho}$ where $\mu=(\rho, \sigma)$ is a double index, $B$ is an $N \times N^{2}$ matrix with components $B(\kappa, \mu)=\delta_{\kappa \sigma}(\mu=(\rho, \sigma))$, and $a$ and $b$ are $N$-component vectors with elements $a_{\kappa}=P(\kappa)$, $\kappa \in \Omega$, and $b_{\kappa}=Q(\kappa), \kappa \in \Omega$, respectively. In addition, the positivity of the quantities $\Pi(\rho, \sigma)$ amounts to the constraint

$$
x \geq 0 \text {. }
$$

Eqs. (45)-(47) are the canonical form of a linear program, which can be solved efficiently in the length $N^{2}$ of the vector $x$. This completes the proof.

It is natural to ask about the properties of the distance in certain simple limiting cases. We consider the following three cases.
Limiting case 1 (Two singleton ensembles). If $P(\rho)=\delta_{\rho \tau}, \rho, \tau \in \Omega$ and $Q(\rho)=\delta_{\rho \sigma}, \rho, \sigma \in \Omega$, i.e., each of the ensembles $P$ and $Q$ consists of only a single state, then the distance between the ensembles is equal to the distance between the respective states,

$$
D^{K}(P, Q)=\Delta(\tau, \sigma) .
$$

Proof. Obviously, the only joint probability distribution with marginals $P$ and $Q$ in this case is $\Pi(\kappa, \tau)=$ $\delta_{\kappa \sigma} \delta_{\tau \rho}$, so the property follows.

Limiting case 2 (One singleton ensemble). If the ensemble $Q(\rho)$ consists of only one state $\sigma$, i.e., $Q(\rho)=$ $\delta_{\rho \sigma}, \rho, \sigma \in \Omega$, then the distance between $P(\rho)$ and $Q(\rho)$ is equal to the average distance between a state drawn from the ensemble $P(\rho)$ and the state $\sigma$,

$$
D^{K}(P, Q)=\sum_{\rho \in \Omega} P(\rho) \Delta(\rho, \sigma) .
$$

Proof. The property follows from the fact that the only joint probability distribution with marginals $P$ and $Q$ in this case is $\Pi(\kappa, \rho)=\delta_{\sigma \kappa} P(\rho)$.

Limiting case 3 (Classical distributions). If the set $\Omega$ consists of perfectly distinguishable density matrices, i.e., $\Delta(\rho, \sigma)=1-\delta_{\rho \sigma}, \forall \rho, \sigma \in \Omega$, then $D^{K}(P, Q)$ reduces to the Kolmogorov distance between the classical probability distributions $P$ and $Q$,

$$
D^{K}(P, Q)=\frac{1}{2} \sum_{\rho \in \Omega}|P(\rho)-Q(\rho)| .
$$

Proof. Since in this case the set $\Omega$ consists of orthogonal states, we can write the right-hand side of Eq. (12) as

$$
\begin{gathered}
\min _{\Pi(\rho, \sigma)} \sum_{\rho, \sigma \in \Omega, \rho \neq \sigma} \Pi(\rho, \sigma) \times 1+\sum_{\rho \in \Omega} \Pi(\rho, \rho) \times 0 \\
=\min _{\Pi(\rho, \sigma)}\left(1-\sum_{\rho \in \Omega} \Pi(\rho, \rho)\right),
\end{gathered}
$$

where the equality follows from the fact that

$$
\sum_{\rho, \sigma \in \Omega, \rho \neq \sigma} \Pi(\rho, \sigma)+\sum_{\rho \in \Omega} \Pi(\rho, \rho)=1 .
$$

The minimum in Eq. (51) is achieved when $\sum_{\rho \in \Omega} \Pi(\rho, \rho)$ is maximal, which in turn is achieved when each of the terms $\Pi(\rho, \rho)$ is maximal. Since the maximum value of $\Pi(\rho, \rho)$ is $\min (P(\rho), Q(\rho))$, we obtain

$$
\begin{gathered}
D^{K}(Q, P)=\left(1-\sum_{\rho \in \Omega} \min (Q(\rho), P(\rho))\right) \\
=\frac{1}{2} \sum_{\rho \in \Omega}|Q(\rho)-P(\rho)| .
\end{gathered}
$$

Comment. Note that we can distinguish two limits which can be interpreted as comparing classical probability distributions. One is Limiting case 3 - probability 
distributions over a set of orthogonal states. The other is the case where each of the two ensembles consists of a single state (two singleton ensembles) and the two states are diagonal in the same basis. In both limits, the distance $D^{K}(Q, P)$ reduces to the Kolmogorov distance between classical distributions.

\section{Properties of the Kantorovich fidelity}

The following properties of the Kantorovich fidelity (13) can be proven similarly to the corresponding properties of the Kantorovich distance, which is why we present them without proof.

Property 1 (Positivity and normalization).

$$
\begin{gathered}
0 \leq F^{K}(P, Q) \leq 1, \\
\forall P, Q \in \mathcal{P}_{\Omega},
\end{gathered}
$$

with

$$
F^{K}(P, Q)=1 \text { iff } P(\rho)=Q(\rho), \forall \rho \in \Omega,
$$

and

$$
F^{K}(P, Q)=0
$$

if and only if the supports of $\mathrm{P}$ and $\mathrm{Q}$ are orthogonal sets of states.

Property 2 (Symmetry).

$$
\begin{gathered}
F^{K}(P, Q)=F^{K}(Q, P), \\
\forall P, Q \in \mathcal{P}_{\Omega} .
\end{gathered}
$$

\section{Property 3 (Joint concavity).}

$$
\begin{gathered}
F^{K}\left(p P_{1}+(1-p) P_{2}, p Q_{1}+(1-p) Q_{2}\right) \\
\geq p F^{K}\left(P_{1}, Q_{1}\right)+(1-p) F^{K}\left(P_{2}, Q_{2}\right), \\
\quad \forall P_{1}, P_{2}, Q_{1}, Q_{2} \in \mathcal{P}_{\Omega}, \quad \forall p \in[0,1] .
\end{gathered}
$$

Property 4 (Monotonicity under CPTP maps). For all CPTP maps $\mathcal{E}$,

$$
F^{K}(P, Q) \leq F^{K}\left(M_{\mathcal{E}}(P), M_{\mathcal{E}}(Q)\right),
$$

where $M_{\mathcal{E}}: \mathcal{P}_{\Omega} \rightarrow \mathcal{P}_{\Omega_{\mathcal{E}}}$ is the map induced by $\mathcal{E}$.

Corollary (Invariance under unitary maps). For all unitary maps $\mathcal{U}$,

$$
F^{K}(P, Q)=F^{K}\left(M_{\mathcal{U}}(P), M_{\mathcal{U}}(Q)\right),
$$

where $M_{\mathcal{U}}: \mathcal{P}_{\Omega} \rightarrow \mathcal{P}_{\Omega_{\mathcal{U}}}$ is the map induced by $\mathcal{U}$.

Property 5 (Monotonicity under averaging). Let $\bar{P}$ denote the singleton ensemble consisting of the average state of $P(\rho), \bar{\rho}_{P}=\sum_{\rho \in \Omega} P(\rho) \rho$. Then

$$
F^{K}(P, Q) \leq F^{K}(\bar{P}, \bar{Q}) .
$$

Corollary. If two distributions are close, their average states are also close, i.e.,

$$
\text { if } F^{K}(P, Q) \geq 1-\varepsilon \text {, then } F\left(\bar{\rho}_{P}, \bar{\rho}_{Q}\right) \geq 1-\varepsilon \text {. }
$$

Property 6 (Stability). Let $P(\rho)$ and $Q(\rho)$ be two ensembles of states in $\Omega$ and $R\left(\sigma^{\prime}\right)$ be an ensemble of states in $\Omega^{\prime}$. Then,

$$
F^{K}(P \otimes R, Q \otimes R)=F^{K}(P, Q) .
$$

Property 7 (Linear programming). The task of finding the optimal $\Pi(\rho, \sigma)$ in Eq. (13) is a linear program and can be solved efficiently in the cardinality of $\Omega$.

Limiting case 1 (Two singleton ensembles). If $P(\rho)=\delta_{\rho \tau}, \rho, \tau \in \Omega$ and $Q(\rho)=\delta_{\rho \sigma}, \rho, \sigma \in \Omega$, i.e., each of the ensembles $P$ and $Q$ consists of only a single state, then the fidelity between the ensembles is equal to the fidelity between the respective states,

$$
F^{K}(P, Q)=F(\tau, \sigma) \text {. }
$$

Limiting case 2 (One singleton ensemble). If the ensemble $Q(\rho)$ consists of only one state $\sigma$, i.e., $Q(\rho)=$ $\delta_{\rho \sigma}, \rho, \sigma \in \Omega$, then the fidelity between $P(\rho)$ and $Q(\rho)$ is equal to the average fidelity between a state drawn from the ensemble $P(\rho)$ and the state $\sigma$,

$$
F^{K}(P, Q)=\sum_{\rho \in \Omega} P(\rho) F(\rho, \sigma)
$$

Limiting case 3 (Classical distributions). If the set $\Omega$ consists of perfectly distinguishable density matrices, i.e., $F(\rho, \sigma)=\delta_{\rho \sigma}, \forall \rho, \sigma \in \Omega$, then $F^{K}(P, Q)$ reduces to the following overlap between the classical probability distributions over the set $\Omega$ :

$$
F^{K}(P, Q)=\sum_{\rho \in \Omega} \min (P(\rho), Q(\rho))=1-\frac{1}{2} \sum_{\rho \in \Omega}|P(\rho)-Q(\rho)| .
$$

Comment. As pointed out earlier, there are two limits which can be interpreted as corresponding to classical probability distributions - Limiting case 3 (probability distributions over a set of orthogonal states), and the limit of two singleton ensembles where the two states are diagonal in the same basis. Here, these two limits yield different results. In the first case, we obtain Eq. (66) which is a particular type of overlap between classical probability distributions. In the second case, if $P(\rho)$ and $Q(\rho)$ are the spectra of the two density matrices, the fidelity reduces to the Bhattacharyya overlap $\sum_{\rho \in \Omega} \sqrt{P(\rho), Q(\rho)}$ which upper bounds expression (66). This reflects the fact that the way $F^{K}$ treats the overlap between the 'classical aspect' of the probability distribution $P(\rho)$ is not a special case of the way it treats the overlap between two quantum states. We will show in subsection E, that this property is intimately related to the fact that $F^{K}$ is not monotonic under measurements. The fidelity which we propose in Sec. V is monotonic under measurements and both its classical limits coincide. 


\section{Operational interpretations of the Kantorovich measures}

To further develop our understanding of the meaning of the Kantorovich measures, it is useful to illustrate their interpretation in the spirit of game theory. Let us consider the Kantorovich distance first.

The trace distance is related to the maximum average probability $p_{\max }(\rho, \sigma)$ with which two equally probable states $\rho$ and $\sigma$ can be distinguished by a measurement as follows: $p_{\max }(\rho, \sigma)=\frac{1}{2}+\frac{1}{2}\|\rho-\sigma\|[6]$. This naturally suggests the following game scenario. Imagine that Alice has access to two ensembles of quantum states $P(\rho)$ and $Q(\rho), \rho \in \Omega$. More precisely, we will assume that she has at her disposal two sufficiently large pools of states in which the relative frequencies of different states are approximately equal to the corresponding probabilities for these states within a satisfactory precision. Alice has to pick one state from one pool and another state from the other pool and choose randomly (with equal probability) whether to send the first state to Bob and throw the other away, or vice versa. She has to tell Bob which is the pair of states drawn from the two ensembles. Bob's task is to distinguish, by performing any operation on the received state, from which ensemble the state he receives has been drawn. This is repeated until the two pools are depleted (the two pools are assumed to have equal numbers of states). Bob's success is measured in terms of the average number of times he guesses correctly the ensemble from which the state he receives has been drawn. Alice's goal, on the other hand, is to choose the pairs of states from the two ensembles in such a way as to make Bob's task as difficult as possible.

If every time Bob employs the optimal measurement strategy for distinguishing which state he has been sent, it is obvious that the optimal strategy for Alice is to pair the states according to the joint probability distribution $\Pi(\sigma, \rho)$ which minimizes the right-hand side of Eq. (12), that is, minimizes the average probability of correctly distinguishing the two states in each pair by an optimal measurement. The Kantorovich distance can then be understood as

$$
D^{K}(P, Q)=2 p_{\max }^{\mathrm{Bob}}(P, Q)-1
$$

where $p_{\max }^{\mathrm{Bob}}(P, Q)$ is Bob's maximal probability of success when Alice chooses her strategy optimally.

The fidelity $F^{K}(P, Q)$ can be given a similar operational interpretation, although a bit more artificial. The difference is that Bob's task and corresponding measure of success have to be chosen so that they are given by the fidelity between the two states which Bob wants to distinguish at every round. For this purpose, we can use Fuchs' operational interpretation of the fidelity [12] as the minimum Bhattacharyya overlap between the statistical distributions generated by all possible measurements on the states,

$$
F(\tau, v)=\min _{\left\{E_{i}\right\}} \sum_{i} \sqrt{\operatorname{Tr}\left(E_{i} \tau\right)} \sqrt{\operatorname{Tr}\left(E_{i} v\right)}
$$

where minimum is taken over all positive operators $\left\{E_{i}\right\}$ that form a positive operator-valued measure $\left(\sum_{i} E_{i}=I\right)$. Then we can modify the game as follows. After sending one of the two states to Bob, Alice does not throw away the other state, but waits for Bob to tell her the type of measurement he performs on his state, and she performs the same measurement on her state. They record their results under many repetitions, and at the end they calculate the average of the statistical overlap between the resulting distributions of measurement outcomes for every pair of states. Bob's task is to minimize this quantity by appropriately choosing his measurements for every pair of states, while Alice's goal is again to make Bob's task as difficult as possible by choosing the pairs of states in a suitable manner.

\section{E. Non-monotonicity under generalized measurements}

The trace distance and the fidelity (as well as all fidelity-based distance measures between states) are monotonic under CPTP maps [2, 20, 32]. This property, also known as contractivity, can be understood as an expression of the fact that the distinguishability between states described by these measures cannot be increased by performing any operation on the states. One may wonder if, when going to the realm of ensembles, we should expect a measure of distinguishability between ensembles to be monotonic under the more general class of stochastic operations, i.e., generalized measurements. After all, these are operations that transform ensembles into ensembles. We will show that this is not satisfied by the Kantorovich distance and fidelity. We will also relate this property to the fact that the Kantorovich fidelity yields two different results in the two 'classical' limits since a necessary condition for a Kantorovich measure to be monotonic under measurements is that both its classical limits are the same. This condition, however, is not sufficient, as shown by the case of the Kantorovich distance.

Note, however, that our definitions of the Kantorovich measures were based on the trace distance and the square root fidelity. In an analogous manner, one can define Kantorovich measures based on any other distance or fidelity between states. Non-monotonicity under generalized measurements is not a problem per se and we will see that there is no reason why we should expect it, considering the operational meaning of the Kantorovich measures based on the trace distance and the square root fidelity. Nevertheless, it would be useful to have measures such that the distinguishability between ensembles that they describe cannot be increased by any possible operation 
(see Sec. V). Driven by this motivation, we derive necessary and sufficient conditions that a measure of distance or fidelity between states has to satisfy in order for the corresponding Kantorovich measure to be monotonic under measurements.

Let us first formulate precisely what we mean by monotonicity under generalized measurements. As pointed out earlier, under the most general type of quantum measurement, the state of a system transforms as in Eq. (3).

Definition 3 (Monotonicity under generalized measurements). Consider a measurement $\mathbf{M}$ with measurement superoperators $\left\{\mathcal{M}_{i}\right\}$. Denote the set of distinct density matrices among all possible outcomes $\frac{\mathcal{M}_{i}(\rho)}{\operatorname{Tr} \mathcal{M}_{i}(\rho)}$ over all possible inputs $\rho \in \Omega$ by $\Omega_{\mathbf{M}}$. If we apply the same generalized measurement (3) to every state in an ensemble $P(\rho), \rho \in \Omega$, we obtain another ensemble $P^{\prime}\left(\rho^{\prime}\right), \rho^{\prime} \in \Omega_{\mathbf{M}}$. Thus the generalized measurement (3) induces a map from the set of probability distributions over $\Omega$ to the set of probability distributions over $\Omega_{\mathbf{M}}$. Denote this map by $M: \mathcal{P}_{\Omega} \rightarrow \mathcal{P}_{\Omega_{\mathrm{M}}}$. When we say that a distance function $D(Q, P)$ between ensembles of states $Q$ and $P$ is monotonically decreasing (or simply monotonic) under generalized measurements, we mean that for any generalized measurement (3),

$$
D(M(P), M(Q)) \leq D(P, Q),
$$

where $M: \mathcal{P}_{\Omega} \rightarrow \mathcal{P}_{\Omega_{\mathbf{M}}}$ is the map induced by the measurement. Similarly, a monotonicity of a fidelity $F(Q, P)$ means

$$
F(M(P), M(Q)) \geq F(P, Q)
$$

for any generalized measurement.

Property. The Kantorovich distance based on the trace distance (Eq. (12) ) and the Kantorovich fidelity based on the square root fidelity (Eq. (13) ) are not monotonic under generalized measurements.

Proof. The proof is presented in Appendix B.

The lack of monotonicity of the Kantorovich measures is something that should not be surprising considering the operational interpretations we discussed in the previous subsection. Generally, monotonicity under certain types of operations means that the type of distinguishability described by the measures cannot be increased under these operations. However, from the above game scenarios we see that the distinguishability concerns Bob's ability do distinguish which of a pair of states Alice has sent to him, in the case where Alice has chosen the way she pairs the states in an optimal way. Certainly, by applying a measurement on the state he receives, Bob cannot improve his chances of guessing correctly beyond what he would obtain by doing the optimal measurement. However, the question of monotonicity we are asking concerns applying the same measurement to all states in the original ensembles before Alice has chosen her optimal strategy. There is no reason to expect that after applying a measurement on all of the states in the original ensembles, the optimal strategy that Alice can employ for the resulting ensembles can only be better than her optimal strategy for the original ensembles. Indeed, as shown in Appendix B, this is not the case when the figure of merit is based on the trace distance or the square root fidelity.

We now provide necessary and sufficient conditions that a measure of distance or fidelity between states has to satisfy in order for the Kantorovich measure based on it to be monotonic under measurements. We will denote by $D_{d}^{K}$ the Kantorovich distance based on a distance $d(\rho, \sigma)$ between states, which is defined as in Eq. (12) with $d$ in the place of $\Delta$. Similarly, by $F_{f}^{K}$ we will denote the Kantorovich fidelity based on a fidelity $f(\rho, \sigma)$ between states.

Theorem 2 (Conditions for monotonicity of the Kantorovich measures under generalized measurements). Let $d(\rho, \sigma)$ and $f(\rho, \sigma)$ be normalized distance and fidelity between states, which are monotonic under CPTP maps and jointly convex (concave). The Kantorovich distance $D_{d}^{K}(P, Q)$ or fidelity $F_{f}^{K}(P, Q)$ based on $d(\rho, \sigma)$ and $f(\rho, \sigma)$, respectively, is monotonic under generalized measurements if and only if for every two states of the form $\sum_{i} p_{i} \rho_{i} \otimes|i\rangle\langle i|$ and $\sum_{i} q_{i} \sigma_{i} \otimes|i\rangle\langle i|$, where $\{|i\rangle\}$ is an orthonormal set of states, the distance and fidelity satisfy

$$
\begin{gathered}
d\left(\sum_{i} p_{i} \rho_{i} \otimes|i\rangle\left\langle i\left|, \sum_{i} q_{i} \sigma_{i} \otimes\right| i\right\rangle\langle i|\right) \\
=\sum_{i}\left(\min \left(p_{i}, q_{i}\right) \Delta\left(\rho_{i}, \sigma_{i}\right)+\frac{1}{2}\left|p_{i}-q_{i}\right|\right),
\end{gathered}
$$

and

$$
\begin{gathered}
f\left(\sum_{i} p_{i} \rho_{i} \otimes|i\rangle\left\langle i\left|, \sum_{i} q_{i} \sigma_{i} \otimes\right| i\right\rangle\langle i|\right) \\
=\sum_{i} \min \left(p_{i}, q_{i}\right) F\left(\rho_{i}, \sigma_{i}\right),
\end{gathered}
$$

respectively.

Proof. The proof is presented in Appendix C.

Comment 1. This theorem is a statement regarding the relation between the values of a given measure (distance or fidelity) between states over Hilbert spaces of different dimensions. Note that if a measure has a well-defined operational interpretation formulated without reference to the dimension of the Hilbert space (to the best of our knowledge, this is the case for all known measures of distance and fidelity between states), that measure is automatically defined for any dimension. The property of monotonicity that we are interested in is also dimension-independent. We remark that the above theorem concerns distance and fidelity measures between states which are monotonic under CPTP maps without the restriction that the CPTP maps preserve the dimension of the Hilbert space since we are interested in proving monotonicity under the most general type of quantum operations. One can easily see that monotonicity under CPTP maps that can increase the dimension is equivalent to monotonicity under dimension-preserving CPTP 
maps plus the stability condition $d(\rho, \sigma)=d(\rho \otimes \kappa, \sigma \otimes \kappa)$ and $f(\rho, \sigma)=f(\rho \otimes \kappa, \sigma \otimes \kappa)$ for all $\rho, \sigma \in \mathcal{B}(\mathcal{H})$ and $\kappa \in \mathcal{B}\left(\mathcal{H}^{\prime}\right)$ where $\mathcal{H}$ and $\mathcal{H}^{\prime}$ are arbitrary Hilbert spaces. Similarly, monotonicity under CPTP maps that can decrease the dimension is equivalent to monotonicity under dimension-preserving CPTP maps plus monotonicity under partial tracing.

Comment 2. The third Jozsa axiom states that a fidelity function should satisfy 37]

$$
f(\rho,|\psi\rangle\langle\psi|)=\langle\psi|\rho| \psi\rangle
$$

The square root fidelity we have considered above satisfies a modified version of that axiom, namely,

$$
F(\rho,|\psi\rangle\langle\psi|)=\sqrt{\langle\psi|\rho| \psi\rangle}
$$

But one can see that if the fidelity $f$ satisfies Eq. (72), it must satisfy

$$
\begin{aligned}
& f\left(\sum_{j} p_{j} \rho_{j} \otimes|j\rangle\langle j|,| \psi\rangle\langle\psi|\otimes| i\rangle\langle i|\right) \\
& =p_{i} f\left(\rho_{i} \otimes|i\rangle\langle i|,| \psi\rangle\langle\psi|\otimes| i\rangle\langle i|\right),
\end{aligned}
$$

which can be only consistent with Eq. (73) and not with Eq. (74). This rules out a class of possible fidelity functions.

A natural question to ask is whether there actually exist measures of distance or fidelity between states that satisfy the conditions of the theorem and thereby would give rise to Kantorovich measures that are monotonic under generalized measurements. We leave this problem open for future investigation. Instead, in the next section we propose distance and fidelity between ensembles which are based on the trace distance and the square root fidelity but are not of the Kantorovich type and satisfy the desired monotonicity.

\section{DISTANCE AND FIDELITY BASED ON THE EXTENDED-HILBERT-SPACE REPRESENTATION OF ENSEMBLES}

\section{A. Motivating the definitions}

In this section, we adopt a different approach to defining measures between ensembles of quantum states, which is based on the extended-Hilbert-space (EHS) representation of ensembles that we briefly touched upon in Sec. II. As we pointed out, an ensemble describes states occurring randomly according to some probability distribution, but an indispensable part of the ensemble is the classical side information about the identity of the given state. The idea behind the EHS representation is that the classical system storing that information is ultimately quantum and therefore it must be possible to describe it in the language of quantum mechanics. In the original formulation of the EHS representation [19], an ensemble of the form $\left\{\left(p_{x}, \rho_{x}\right)\right\}$ is represented in terms of a state of the form $\hat{\rho}=\sum_{x} p_{x} \rho_{x} \otimes[x]($ Eq. (8) $)$. When only a single ensemble is involved, this representation is sufficient and it is not important what the pointer (or flag) states $[x] \equiv|x\rangle\langle x|$ are, as long as they form an orthonormal set and each $[x]$ is unambiguously associated with $\rho_{x}$. However, if we want to use the EHS idea to compare two ensembles, we need to go beyond this simple formulation. In Sec. III, we already saw one example where a naive application of this idea fails. Namely, we argued that if we represent two ensembles $P(\rho)$ and $Q(\rho), \rho \in \Omega$, by the states $\sum_{\rho \in \Omega} P(\rho) \rho \otimes[\rho]$ and $\sum_{\rho \in \Omega} Q(\rho) \rho \otimes[\rho]$, a distance or fidelity between these EHS representations is equivalent to a distance or fidelity between the probability distributions $P(\rho)$ and $Q(\rho)$ in which $\rho$ is treated as a classical variable. Such a measure does not capture the idea of closeness between different quantum states. In this section, we will provide a generalized formulation of an EHS representation of an ensemble, which will allow us to define measures of distance and fidelity between ensembles that possess all properties that we would like such measures to have.

For this purpose, it is convenient to introduce the notion of a 'classical' system whose states live in a 'classical' space which we define to be a fixed set $\Omega^{C}$ of orthogonal pure states $[c], \operatorname{Tr}\left([c]\left[c^{\prime}\right]\right)=\delta_{c c^{\prime}}$, where we use the notation $[c] \equiv|c\rangle\langle c|$ to distinguish the states of the 'classical' system from the states of the quantum system. Generally, the classical space can consist of infinitely many different states, but later we will see that it suffices to consider a classical space of cardinality $\left|\Omega^{C}\right|=|\Omega|^{2}$, where $|\Omega|$ is the cardinality of the set $\Omega$ of density matrices participating in the ensembles.

Given the classical system described by the classical space $\Omega^{C}$ and a set $\Omega$ of states of a quantum system, we can ask what are the most general states of the quantumclassical system that represent an ensemble $P(\rho), \rho \in \Omega$, consistently with our notion of ensemble. As we pointed out, the information about the identity of a quantum state from the ensemble must be stored in the classical system in a way which allows one to unambiguously identify the state by measuring the state of the classical system. If we take this to be the definition of a valid EHS representation, then we should allow for the possibility that several flag states $\left\{\left[c_{i}(\rho)\right]\right\}$ point at the same quantum state as long as every flag state is associated with a single quantum state and, of course, each quantum state $\rho$ still appears with the correct total probability. More succinctly, the most general EHS representation should allow for mixed flag states, i.e.,

$$
\widehat{\rho}_{P}=\sum_{\rho \in \Omega} P(\rho) \rho \otimes\left(\sum_{i} p_{i}(\rho)\left[c_{i}(\rho)\right]\right) .
$$

Having a quantum-classical state of this form is equivalent to having the ensemble $\{(P(\rho), \rho)\}$ because by measuring the state of the classical system, we can infer which state from the ensemble we are given, and given a state drawn randomly from the ensemble we can always pre- 
pare the state (76) by attaching the corresponding classical state and discarding any additional information. Note that in the expression (76) we have written the classical states as $\left[c_{i}(\rho)\right]$, explicitly indicating which classical states are associated with the quantum state $\rho$, but it is convenient to express the condition that every pointer state is associated with a unique $\rho \in \Omega$ as a condition on a general state of the quantum-classical system.

Definition 4 (EHS representation of an ensemble). An EHS representation of an ensemble $P(\rho)$, $\rho \in \Omega$, is a quantum-classical state of the form

$$
\widehat{\rho}=\sum_{\rho \in \Omega} \sum_{[c] \in \Omega^{C}} \widetilde{P}(\rho,[c]) \rho \otimes[c]
$$

for which the non-negative quantities $\widetilde{P}(\rho,[c])$ satisfy

$$
\begin{gathered}
\sum_{[c] \in \Omega^{C}} \widetilde{P}(\rho,[c])=P(\rho), \quad \forall \rho \in \Omega, \\
\widetilde{P}(\rho,[c]) \widetilde{P}(\sigma,[c])=0, \quad \forall \rho, \sigma \in \Omega \mid \rho \neq \sigma, \quad \forall[c] \in \Omega^{C} .
\end{gathered}
$$

Equation (78) ensures that every quantum state $\rho \in \Omega$ occurs with the correct probability $P(\rho)$ and Eq. (79) expresses the fact that a given pointer state $[c]$ in $\Omega^{C}$ cannot be associated with more than one state in $\Omega$. In other words, there exists an injective function $\zeta: \Omega \rightarrow \Omega^{C}$ which specifies the pointer states associated with a given $\rho \in \Omega$, and $\widetilde{P}(\rho,[c])=0$ if $\zeta^{-1}([c]) \neq \rho$. It is important to note that a given ensemble can be encoded using many different injections. If two ensembles $P$ and $Q$ are encoded using injections $\zeta_{P}$ and $\zeta_{Q}$ which map the space $\Omega$ to two non-overlapping subsets of $\Omega^{C}$, the corresponding EHS representations of the two ensembles would be completely orthogonal and therefore perfectly distinguishable. However, if the sets of quantum states participating in the two ensembles are not orthogonal, one can always chose two EHS representations of the two ensembles which have a non-zero overlap because one can assign one and the same pointer to two non-overlapping states from the two ensembles. At the same time, unless the two ensembles are identical, their EHS representations cannot be made identical. This suggests a way of defining distance and fidelity between ensembles based on an optimal choice of their EHS representations.

Definition 5 (EHS distance between ensembles). The EHS distance between the ensembles $P(\rho)$ and $Q(\rho)$, $\rho \in \Omega$, is

$$
D^{\mathrm{EHS}}(P, Q)=\min _{\widehat{\rho}, \widehat{\sigma}} \Delta(\widehat{\rho}, \widehat{\sigma}),
$$

where $\Delta$ is the trace distance (Eq. (2)), and minimum is taken over all EHS representations $\widehat{\rho}$ and $\widehat{\sigma}$ of $P(\rho)$ and $Q(\rho)$, respectively.

Definition 6 (EHS fidelity between ensembles). The EHS fidelity between the ensembles $P(\rho)$ and $Q(\rho)$, $\rho \in \Omega$, is

$$
F^{\mathrm{EHS}}(P, Q)=\max _{\widehat{\rho}, \widehat{\sigma}} F(\widehat{\rho}, \widehat{\sigma})
$$

where $F$ is the square root fidelity (Eq. (1)), and maximum is taken over all EHS representations $\widehat{\rho}$ and $\widehat{\sigma}$ of $P(\rho)$ and $Q(\rho)$, respectively.

Before we proceed with studying the properties of these measures, it is convenient to present two equivalent formulations of the above definitions.

Lemma 2 (Equivalent form of the EHS distance). The EHS distance (80) is equivalent to

$$
\begin{gathered}
D^{\mathrm{EHS}}(P, Q)= \\
\min _{\substack{P(\rho, \sigma), Q(\rho, \sigma)}} \Delta\left(\sum_{\rho, \sigma \in \Omega} P(\rho, \sigma) \rho \otimes[\rho \sigma], \sum_{\rho, \sigma \in \Omega} Q(\rho, \sigma) \sigma \otimes[\rho \sigma]\right),
\end{gathered}
$$

where minimum is taken over pairs of joint probability distributions $P(\rho, \sigma)$ and $Q(\rho, \sigma)$ such that the left marginal of $P(\rho, \sigma)$ is equal to $P(\rho)$ and the right marginal of $Q(\rho, \sigma)$ is equal to $Q(\sigma)$. The set of pointer states $[\rho \sigma]$ is fixed and has cardinality equal to the square of the cardinality of $\Omega$.

Proof. First, observe that for any two EHS representations $\widehat{\rho}$ and $\widehat{\sigma}$ of $P$ and $Q$, the distance $\Delta(\widehat{\rho}, \widehat{\sigma})$ has the form

$$
\begin{gathered}
\Delta(\widehat{\rho}, \widehat{\sigma})= \\
\Delta\left(\sum_{\rho \in \Omega} \sum_{[c] \in \Omega^{C}} \widetilde{P}(\rho,[c]) \rho \otimes[c], \sum_{\rho \in \Omega} \sum_{[c] \in \Omega^{C}} \widetilde{Q}(\rho,[c]) \rho \otimes[c]\right),
\end{gathered}
$$

where $\widetilde{P}(\rho,[c])$ and $\widetilde{Q}(\rho,[c])$ are consistent with Definition 4 . It can generally happen that one and the same pointer $[c]$ is attached to a state $\rho$ from the first ensemble and to a state $\sigma$ from the second ensemble, that is, $\widetilde{P}(\rho,[c]) \neq 0$ and $\widetilde{Q}(\sigma,[c]) \neq 0$. However, having a pair of states $\rho$ and $\sigma$ from the first and second ensembles, respectively, attached simultaneously to more than one pointer, does not help in attaining the minimum in Eq. (80). This follows from the fact that we could replace the second pointer by the first one, which would result in valid EHS representations of the two ensembles. But the latter operation also corresponds to a CPTP map on the states in the extended Hilbert space, and since $\Delta$ is monotonic under CPTP maps, the resultant representations will be closer. Therefore, without loss of generality, we can assume that every pair of states $\rho$ and $\sigma$ from the first and second ensemble, respectively, is associated with a single pointer state, which we will label by $[\rho \sigma]$. This implies that the minimum in Eq. (80) can be taken over EHS representations of $P$ and $Q$ of the form $\sum_{\rho, \sigma \in \Omega} P(\rho, \sigma) \rho \otimes[\rho \sigma]$ and $\sum_{\rho, \sigma \in \Omega} Q(\rho, \sigma) \sigma \otimes[\rho \sigma]$, where the condition of consistency with the original distributions $P$ and $Q$ amounts to conditions on the left and right marginals of $P(\rho, \sigma)$ and $Q(\rho, \sigma)$, respectively:

$$
\begin{aligned}
& \sum_{\sigma} P(\rho, \sigma)=P(\rho), \\
& \sum_{\rho} Q(\rho, \sigma)=Q(\sigma) .
\end{aligned}
$$

This completes the proof. 
Lemma 3 (Equivalent form of the EHS fidelity). The EHS fidelity (81) is equivalent to

$F^{\mathrm{EHS}}(P, Q)=$
$\max _{P(\rho, \sigma), Q(\rho, \sigma)} F\left(\sum_{\rho, \sigma \in \Omega} P(\rho, \sigma) \rho \otimes[\rho \sigma], \sum_{\rho, \sigma \in \Omega} Q(\rho, \sigma) \sigma \otimes[\rho \sigma]\right)$,

where minimum is taken over pairs of joint probability distributions $P(\rho, \sigma)$ and $Q(\rho, \sigma)$ such that the left marginal of $P(\rho, \sigma)$ is equal to $P(\rho)$ and the right marginal of $Q(\rho, \sigma)$ is equal to $Q(\sigma)$. The set of pointer states $[\rho \sigma]$ is fixed and has cardinality equal to the square of the cardinality of $\Omega$.

Proof. The proof is analogous to the proof of Lemma 2.

Corollary (Formulation without reference to an extended Hilbert space). Considering the explicit forms of the trace distance and the square root fidelity, one can see that Eqs. (82) and (86) can be written without reference to the classical pointer system:

$D^{\mathrm{EHS}}(P, Q)=\frac{1}{2} \min _{P(\rho, \sigma), Q(\rho, \sigma)} \sum_{\rho, \sigma \in \Omega}\|P(\rho, \sigma) \rho-Q(\rho, \sigma) \sigma\|$,

$F^{\mathrm{EHS}}(P, Q)=\max _{P(\rho, \sigma), Q(\rho, \sigma)} \sum_{\rho, \sigma \in \Omega} \sqrt{P(\rho, \sigma) Q(\rho, \sigma)} F(\rho, \sigma)$,

where optimization is taken over all joint distributions $P(\rho, \sigma)$ with left marginal $P(\rho)$ and $Q(\rho, \sigma)$ with right marginal $Q(\sigma)$.

\section{B. Properties of the EHS distance}

\section{Property 1 (Positivity).}

$$
\begin{gathered}
D^{\mathrm{EHS}}(P, Q) \geq 0, \\
\forall P, Q \in \mathcal{P}_{\Omega},
\end{gathered}
$$

with equality

$$
D^{\mathrm{EHS}}(P, Q)=0 \text { iff } P(\rho)=Q(\rho), \forall \rho \in \Omega .
$$

Proof. The EHS distance is obviously non-negative since $\Delta(\rho, \sigma) \geq 0$. If both ensembles are the same, $P(\rho)=Q(\rho), \forall \rho \in \Omega$, clearly $D^{\mathrm{EHS}}(P, Q)=0$, because we can choose identical EHS representations for both ensembles. Reversely, if $D^{\mathrm{EHS}}(P, Q)=0$, this means that the EHS representations of $P$ and $Q$ must be identical, which means that $P$ and $Q$ must be the same.

Property 2 (Normalization).

$$
\begin{gathered}
D^{\mathrm{EHS}}(P, Q) \leq 1, \\
\forall P, Q \in \mathcal{P}_{\Omega},
\end{gathered}
$$

with equality

$$
D^{\mathrm{EHS}}(P, Q)=1
$$

if and only if the supports of $\mathrm{P}$ and $\mathrm{Q}$ are orthogonal sets of states.

Proof. Since $\Delta(\rho, \sigma) \leq 1$, obviously $D^{\mathrm{EHS}}(P, Q) \leq 1$. If $P$ and $Q$ have supports on orthogonal sets of states, then all of their EHS representations will also be orthogonal, which implies $D^{\mathrm{EHS}}(P, Q)=1$. Reversely, if $D^{\mathrm{EHS}}(P, Q)=1$, this means that the EHS states for which the minimum in Eq. (800) is achieved, must be orthogonal. But unless $P$ and $Q$ have supports on orthogonal sets of states, it is always possible to find EHS representations of $P$ and $Q$ which have non-zero overlap because we can assign one and the same pointer to two non-overlapping states from the two different ensembles.

Property 3 (Symmetry).

$$
\begin{gathered}
D^{\mathrm{EHS}}(P, Q)=D^{\mathrm{EHS}}(Q, P), \\
\forall P, Q \in \mathcal{P}_{\Omega} .
\end{gathered}
$$

Proof. The symmetry follows from the definition (12) and the symmetry of $\Delta(\rho, \sigma)$.

Property 4 (Triangle inequality).

$$
\begin{aligned}
D^{\mathrm{EHS}}(P, R) \leq & D^{\mathrm{EHS}}(P, Q)+D^{\mathrm{EHS}}(Q, R), \\
& \forall P, Q, R \in \mathcal{P}_{\Omega} .
\end{aligned}
$$

Proof. The proof is presented in Appendix D.

Property 5 (Joint convexity).

$$
\begin{gathered}
D^{\mathrm{EHS}}\left(p P_{1}+(1-p) P_{2}, p Q_{1}+(1-p) Q_{2}\right) \\
\leq p D^{\mathrm{EHS}}\left(P_{1}, Q_{1}\right)+(1-p) D^{\mathrm{EHS}}\left(P_{2}, Q_{2}\right), \\
\quad \forall P_{1}, P_{2}, Q_{1}, Q_{2} \in \mathcal{P}_{\Omega}, \quad \forall p \in[0,1] .
\end{gathered}
$$

Proof. Let

$$
\begin{gathered}
D^{\mathrm{EHS}}\left(P_{1}, Q_{1}\right)= \\
\Delta\left(\sum_{\rho, \sigma \in \Omega} P_{1}(\rho, \sigma) \rho \otimes[\rho \sigma], \sum_{\rho, \sigma \in \Omega} Q_{1}(\rho, \sigma) \sigma \otimes[\rho \sigma]\right)
\end{gathered}
$$

and

$$
\begin{gathered}
D^{\mathrm{EHS}}\left(P_{2}, Q_{2}\right)= \\
\Delta\left(\sum_{\rho, \sigma \in \Omega} P_{2}(\rho, \sigma) \rho \otimes[\rho \sigma], \sum_{\rho, \sigma \in \Omega} Q_{2}(\rho, \sigma) \sigma \otimes[\rho \sigma]\right),
\end{gathered}
$$

where the joint distributions $P_{1}(\rho, \sigma)$ and $P_{2}(\rho, \sigma)$ have left marginals $P_{1}(\rho)$ and $P_{2}(\rho)$, respectively, and the joint distributions $Q_{1}(\rho, \sigma)$ and $Q_{2}(\rho, \sigma)$ have right marginals $Q_{1}(\sigma)$ and $Q_{2}(\sigma)$, respectively. Since $\Delta$ is jointly convex, we have

$$
\begin{gathered}
p D^{\mathrm{EHS}}\left(P_{1}, Q_{1}\right)+(1-p) D^{\mathrm{EHS}}\left(P_{2}, Q_{2}\right) \geq \\
\Delta\left(\sum_{\rho, \sigma \in \Omega}\left(p P_{1}(\rho, \sigma)+(1-p) P_{2}(\rho, \sigma)\right) \rho \otimes[\rho \sigma],\right. \\
\left.\sum_{\rho, \sigma \in \Omega}\left(p Q_{1}(\rho, \sigma)+(1-p) Q_{2}(\rho, \sigma)\right) \sigma \otimes[\rho \sigma]\right) .
\end{gathered}
$$


But obviously $p P_{1}(\rho, \sigma)+(1-p) P_{2}(\rho, \sigma)$ is a joint distribution with left marginal $p P_{1}(\rho)+(1-p) P_{2}(\rho)$, and $p Q_{1}(\rho, \sigma)+(1-p) Q_{2}(\rho, \sigma)$ is a joint distribution with right marginal $p Q_{1}(\sigma)+(1-p) Q_{2}(\sigma)$. Therefore, the quantity on the right-hand side of Eq. (99) is greater than or equal to $D^{\mathrm{EHS}}\left(p P_{1}+(1-p) P_{2}, p Q_{1}+(1-p) Q_{2}\right)$, which completes the proof.

Property 6 (Monotonicity under generalized measurements). $D^{\mathrm{EHS}}(P, Q)$ is monotonic under generalized measurements in the sense of Definition 3, $D^{\mathrm{EHS}}(P, Q) \geq D^{\mathrm{EHS}}(M(P), M(Q))$.

Proof. Let $P(\rho)$ and $Q(\rho), \rho \in \Omega$ be two ensembles of quantum states, and let

$$
\begin{gathered}
D^{\mathrm{EHS}}(P, Q)= \\
\Delta\left(\sum_{\rho, \sigma \in \Omega} P(\rho, \sigma) \rho \otimes[\rho \sigma], \sum_{\rho, \sigma \in \Omega} Q(\rho, \sigma) \sigma \otimes[\rho \sigma]\right) .
\end{gathered}
$$

Let $\left\{\mathcal{M}_{i}\right\}, \mathcal{M}_{i}(\rho)=\sum_{j} M_{i j} \rho M_{i j}^{\dagger}$, be the measurement superoperators of a generalized measurement $\mathbf{M}$, $\sum_{i, j} M_{i j}^{\dagger} M_{i j}=I$. Consider the following CPTP map:

$$
\mathcal{M}(\rho) \rightarrow \sum_{i} \mathcal{M}_{i}(\rho) \otimes[i],
$$

where $\{[i]\}$ is an orthonormal set of pure states in the Hilbert space of some additional system. Since $\Delta$ is monotonic under CPTP maps, we have

$$
\begin{gathered}
D^{\mathrm{EHS}}(P, Q) \geq \\
\Delta\left(\mathcal{M}\left(\sum_{\rho, \sigma \in \Omega} P(\rho, \sigma) \rho \otimes[\rho \sigma]\right), \mathcal{M}\left(\sum_{\rho, \sigma \in \Omega} Q(\rho, \sigma) \sigma \otimes[\rho \sigma]\right)\right) \\
=\Delta\left(\sum_{\rho, \sigma \in \Omega} \sum_{i} P(\rho, \sigma) \operatorname{Tr}\left(\mathcal{M}_{i}(\rho)\right) \frac{\mathcal{M}_{i}(\rho)}{\operatorname{Tr}\left(\mathcal{M}_{i}(\rho)\right)} \otimes[\rho \sigma i],\right. \\
\left.\sum_{\rho, \sigma \in \Omega} \sum_{i} Q(\rho, \sigma) \operatorname{Tr}\left(\mathcal{M}_{i}(\sigma)\right) \frac{\mathcal{M}_{i}(\sigma)}{\operatorname{Tr}\left(\mathcal{M}_{i}(\sigma)\right)} \otimes[\rho \sigma i]\right) \\
\geq D^{\mathrm{EHS}}(M(P), M(Q)),
\end{gathered}
$$

where $M: \mathcal{P}_{\Omega} \rightarrow \mathcal{P}_{\Omega_{\mathrm{M}}}$ is the map induced by the measurement as explained in Definition 3. The last inequality follows from the fact that $\sum_{\rho, \sigma \in \Omega} \sum_{i} P(\rho, \sigma) \operatorname{Tr}\left(\mathcal{M}_{i}(\rho)\right) \frac{\mathcal{M}_{i}(\rho)}{\operatorname{Tr}\left(\mathcal{M}_{i}(\rho)\right)} \otimes[\rho \sigma i]$ and $\sum_{\rho, \sigma \in \Omega} \sum_{i} Q(\rho, \sigma) \operatorname{Tr}\left(\mathcal{M}_{i}(\sigma)\right) \frac{\mathcal{M}_{i}(\sigma)}{\operatorname{Tr}\left(\mathcal{M}_{i}(\sigma)\right)} \otimes[\rho \sigma i]$ are EHS representations of the new ensembles $M(P)$ and $M(Q)$.

Corollary (Monotonicity under CPTP maps and invariance under unitary maps). Property 6 obviously implies monotonicity under CPTP maps, which can be regarded as a special type of generalized measurements. This in turn implies invariance under unitary maps since the latter are reversible CPTP maps.

Property 7 (Monotonicity under averaging). Let $\bar{P}$ denote the singleton ensemble consisting of the average state of $P(\rho), \bar{\rho}_{P}=\sum_{\rho \in \Omega} P(\rho) \rho$. Then

$$
D^{\mathrm{EHS}}(P, Q) \geq D^{\mathrm{EHS}}(\bar{P}, \bar{Q}) .
$$

Proof. Let

$$
\begin{gathered}
D^{\mathrm{EHS}}(P, Q)= \\
\Delta\left(\sum_{\rho, \sigma \in \Omega} P(\rho, \sigma) \rho \otimes[\rho \sigma], \sum_{\rho, \sigma \in \Omega} Q(\rho, \sigma) \sigma \otimes[\rho \sigma]\right) .
\end{gathered}
$$

Observe that

$$
\bar{\rho}_{P}=\operatorname{Tr}_{C}\left(\sum_{\rho, \sigma \in \Omega} P(\rho, \sigma) \rho \otimes[\rho \sigma]\right)
$$

and

$$
\bar{\rho}_{Q}=\operatorname{Tr}_{C}\left(\sum_{\rho, \sigma \in \Omega} Q(\rho, \sigma) \sigma \otimes[\rho \sigma]\right),
$$

where $\operatorname{Tr}_{C}$ denotes partial tracing over the subsystem containing the classical pointers $\{[\rho \sigma]\}$. On the other hand, $\Delta(\bar{\rho}, \bar{\sigma})=D^{\mathrm{EHS}}(\bar{P}, \bar{Q})$ (see Eq. (116) below). Since $\Delta(\rho, \sigma)$ is monotonic under partial tracing (which is a CPTP map), the property follows.

Corollary. If two distributions are close, their average states are also close, i.e.,

$$
\text { if } D^{\mathrm{EHS}}(P, Q) \leq \varepsilon \text {, then } \Delta\left(\bar{\rho}_{P}, \bar{\rho}_{Q}\right) \leq \varepsilon \text {. }
$$

Property 8 (Continuity of the average of a continuous function). Let $h(\rho)$ be a bounded function, which is continuous with respect to the distance $\Delta$. Then the ensemble average of $h(\rho), \bar{h}_{P}=\sum_{\rho \in \Omega} P(\rho) h(\rho)$, is continuous with respect to $D^{\mathrm{EHS}}$.

Proof. The proof is presented in Appendix E.

Comment. Again, as we pointed out in relation to the Kantorovich distance, Property 8 naturally reflects the idea of states as resources - if a resource is a continuous function of the state, when two ensembles are close, their average resources must also be close.

Property 9 (The EHS distance is upper bounded by the Kantorovich distance).

$$
D^{\mathrm{EHS}}(P, Q) \leq D^{K}(P, Q) .
$$

Proof. Let $\Pi(\rho, \sigma)$ be a joint probability distribution with left and right marginals $P(\rho)$ and $Q(\sigma)$ for which the minimum in the definition (12) of $D^{K}(P, Q)$ is attained. Obviously, the minimum in Eq. (82) satisfies

$$
\begin{aligned}
& D^{\mathrm{EHS}}(P, Q) \leq \\
& \Delta\left(\sum_{\rho, \sigma \in \Omega} \Pi(\rho, \sigma) \rho \otimes[\rho \sigma], \sum_{\rho, \sigma \in \Omega} \Pi(\rho, \sigma) \sigma \otimes[\rho \sigma]\right) \\
& =\sum_{\rho, \sigma \in \Omega} \Pi(\rho, \sigma) \Delta(\rho, \sigma)=D^{K}(P, Q) .
\end{aligned}
$$

Property 10 (Stability). Let $P(\rho)$ and $Q(\rho)$ be two ensembles of states in $\Omega$ and $R\left(\sigma^{\prime}\right)$ be an ensemble of states in $\Omega^{\prime}$, where $\Omega$ and $\Omega^{\prime}$ are sets of states of two different systems. Then,

$$
D^{\mathrm{EHS}}(P \otimes R, Q \otimes R)=D^{\mathrm{EHS}}(P, Q) .
$$


Proof. Let

$$
\begin{gathered}
D^{\mathrm{EHS}}(P \otimes R, Q \otimes R)= \\
\Delta\left(\sum_{\rho, \sigma \in \Omega} \sum_{\tau^{\prime}, \kappa^{\prime} \in \Omega^{\prime}} \Pi\left(\rho \otimes \tau^{\prime}, \sigma \otimes \kappa^{\prime}\right) \rho \otimes \tau^{\prime} \otimes\left[\rho \tau^{\prime} \sigma \kappa^{\prime}\right],\right. \\
\left.\sum_{\rho, \sigma \in \Omega} \sum_{\tau^{\prime}, \kappa^{\prime} \in \Omega^{\prime}} J\left(\rho \otimes \tau^{\prime}, \sigma \otimes \kappa^{\prime}\right) \sigma \otimes \kappa^{\prime} \otimes\left[\rho \tau^{\prime} \sigma \kappa^{\prime}\right]\right),
\end{gathered}
$$

where $\Pi\left(\rho \otimes \tau^{\prime}, \sigma \otimes \kappa^{\prime}\right)$ has left marginal $P(\rho) R\left(\tau^{\prime}\right)$ and $J\left(\rho \otimes \tau^{\prime}, \sigma \otimes \kappa^{\prime}\right)$ has right marginal $Q(\sigma) R\left(\tau^{\prime}\right)$.

One can readily see that the monotonicity of $\Delta$ under partial tracing implies

$$
D^{\mathrm{EHS}}(P \otimes R, Q \otimes R) \geq D^{\mathrm{EHS}}(P, Q) .
$$

Using the stability of $\Delta$, we see that if we choose $\Pi\left(\rho \otimes \tau^{\prime}, \sigma \otimes \kappa^{\prime}\right)=P(\rho, \sigma) R\left(\tau^{\prime}\right) \delta_{\tau^{\prime} \kappa^{\prime}}$ and $J\left(\rho \otimes \tau^{\prime}, \sigma \otimes \kappa^{\prime}\right)=$ $Q(\rho, \sigma) R\left(\tau^{\prime}\right) \delta_{\tau^{\prime} \kappa^{\prime}}$, where $P(\rho, \sigma)$ and $Q(\rho, \sigma)$ are two joint distributions for which the minimum in Eq. (82) is attained, we obtain

$$
D^{\mathrm{EHS}}(P \otimes R, Q \otimes R) \leq D^{\mathrm{EHS}}(P, Q),
$$

which together with Eq. (112) implies Eq. (110).

This property can also be seen to follow from Property 6 because one can go from $P$ and $Q$ to $P \otimes R$ and $Q \otimes R$, respectively, and vice versa, via stochastic operations.

Property 11 (Convex optimization). The task of finding the optimal $P(\rho, \sigma)$ and $Q(\rho, \sigma)$ in Eq.(82) is a convex optimization problem.

Proof. We can think of $P(\rho, \sigma)$ and $Q(\rho, \sigma)$ as the components of a vector $x$ of dimension $2 N^{2}$, where $N$ is the cardinality of the set $\Omega$. The first $N^{2}$ components of the vector are equal to $P(\rho, \sigma)$ and the second $N^{2}$ components are equal to $Q(\rho, \sigma)$. The convexity of the function

$$
\xi(x) \equiv \Delta\left(\sum_{\rho, \sigma \in \Omega} P(\rho, \sigma) \rho \otimes[\rho \sigma], \sum_{\rho, \sigma \in \Omega} Q(\rho, \sigma) \sigma \otimes[\rho \sigma]\right)
$$

can be seen from the fact that for any $x_{1}, x_{2}$, and $t$, $0 \leq t \leq 1$, we have

$$
\begin{gathered}
\xi\left(t x_{1}+(1-t) x_{2}\right)= \\
\Delta\left(\sum_{\rho, \sigma \in \Omega}\left(t P_{1}(\rho, \sigma)+(1-t) P_{2}(\rho, \sigma)\right) \rho \otimes[\rho \sigma],\right. \\
\left.\sum_{\rho, \sigma \in \Omega}\left(t Q_{1}(\rho, \sigma)+(1-t) Q_{2}(\rho, \sigma)\right) \sigma \otimes[\rho \sigma]\right) \leq \\
t \Delta\left(\sum_{\rho, \sigma \in \Omega} P_{1}(\rho, \sigma) \rho \otimes[\rho \sigma], \sum_{\rho, \sigma \in \Omega} Q_{1}(\rho, \sigma) \sigma \otimes[\rho \sigma]\right)+ \\
(1-t) \Delta\left(\sum_{\rho, \sigma \in \Omega} P_{2}(\rho, \sigma) \rho \otimes[\rho \sigma], \sum_{\rho, \sigma \in \Omega} Q_{2}(\rho, \sigma) \sigma \otimes[\rho \sigma]\right) \\
=t \xi\left(x_{1}\right)+(1-t) \xi\left(x_{2}\right),
\end{gathered}
$$

due to the joint convexity of $\Delta$. Notice that if $P_{1}(\rho, \sigma)$ and $P_{2}(\rho, \sigma)$ have left marginals equal to $P(\rho)$, so does $t P_{1}(\rho, \sigma)+(1-t) P_{2}(\rho, \sigma)$. Similarly, if $Q_{1}(\rho, \sigma)$ and $Q_{2}(\rho, \sigma)$ have right marginals equal to $Q(\rho)$, so does $t Q_{1}(\rho, \sigma)+(1-t) Q_{2}(\rho, \sigma)$. Since the marginal conditions on $x$ are linear, the problem of finding $x$ which minimizes $\xi(x)$ subject to these constraints is a convex optimization problem, for which efficient numerical techniques exist.

Limiting case 1 (Two singleton ensembles). If $P(\rho)=\delta_{\rho \tau}, \rho, \tau \in \Omega$ and $Q(\rho)=\delta_{\rho \sigma}, \rho, \sigma \in \Omega$, i.e., each of the ensembles $P$ and $Q$ consists of only a single state, then the distance between the ensembles is equal to the distance between the respective states,

$$
D^{\mathrm{EHS}}(P, Q)=\Delta(\tau, \sigma) .
$$

Proof. Due to the monotonicity of $\Delta$ under partial tracing over the pointer system, we have that $D(P, Q) \geq$ $\Delta(\tau, \sigma)$. But clearly, equality is achievable because we can choose the probability distributions in Eq. (82) $P(\kappa, \rho)=Q(\kappa, \rho)=\delta_{\kappa \tau} \delta_{\rho \sigma}$.

Limiting case 2 (One singleton ensemble). Unlike the Kantorovich distance, when the ensemble $Q(\rho)$ consists of only one state $\sigma$, i.e., $Q(\rho)=\delta_{\rho \sigma}, \rho, \sigma \in \Omega$, the EHS distance between $P(\rho)$ and $Q(\rho)$ is generally not equal to the average distance between a state drawn from the ensemble $P(\rho)$ and the state $\sigma$,

$$
D^{\mathrm{EHS}}(P, Q) \neq \sum_{\rho \in \Omega} P(\rho) \Delta(\rho, \sigma) .
$$

Proof. We provide a proof by counterexample. Let the singleton ensemble consist of the sate $\sigma_{0}=\tilde{\sigma} \otimes|0\rangle\langle 0|$ and let the other ensemble consist of two states, $\rho_{0}=$ $\tilde{\rho}_{0} \otimes|0\rangle\langle 0|$ and $\rho_{1}=\tilde{\rho}_{1} \otimes|1\rangle\langle 1|$, with probabilities $p_{0}$ and $p_{1}=1-p_{0}$, respectively. The average distance between the state $\sigma_{0}$ and the states from the other ensemble is

$$
\begin{aligned}
\Delta_{a v e}= & p_{0} \Delta\left(\rho_{0}, \sigma_{0}\right)+p_{1} \Delta\left(\rho_{1}, \sigma_{0}\right) \\
& =p_{0} \Delta\left(\tilde{\rho}_{0}, \tilde{\sigma}_{0}\right)+p_{1} .
\end{aligned}
$$

However, if we choose the joint distributions $P(\rho, \sigma)=$ $p_{0} \delta_{\rho \rho_{0}}+p_{1} \delta_{\rho \rho_{1}}$ and $Q(\rho, \sigma)=\delta_{\rho_{0} \sigma_{0}}$, we see from Eq. (87) that

$$
\begin{gathered}
D^{\mathrm{EHS}}(P, Q) \leq \frac{1}{2}\left\|p_{0} \rho_{0}-\sigma_{0}\right\|+\frac{1}{2} p_{1} \leq \\
\frac{p_{0}}{2}\left\|\rho_{0}-\sigma_{0}\right\|+\frac{1}{2}\left(1-p_{0}\right)\left\|\sigma_{0}\right\|+\frac{1}{2} p_{1}= \\
p_{0} \frac{1}{2}\left\|\tilde{\rho}_{0}-\tilde{\sigma}_{0}\right\|+p_{1}=\Delta_{\text {ave }} .
\end{gathered}
$$

For an appropriate choice of $\tilde{\rho}_{0}$ and $\tilde{\sigma_{0}}$, the second inequality can be made strict, which completes the proof.

Limiting case 3 (Classical distributions). If the set $\Omega$ consists of perfectly distinguishable density matrices, i.e., $\Delta(\rho, \sigma)=1-\delta_{\rho \sigma}, \forall \rho, \sigma \in \Omega$, then $D^{\mathrm{EHS}}(P, Q)$ reduces to the trace distance $\Delta\left(\bar{\rho}_{P}, \bar{\rho}_{Q}\right)$ between the density matrices $\bar{\rho}_{P}=\sum_{\rho \in \Omega} P(\rho) \rho$ and $\bar{\rho}_{Q}=\sum_{\rho \in \Omega} Q(\rho) \rho$, which is equal to the Kolmogorov distance between the classical probability distributions $P$ and $Q, D^{\mathrm{EHS}}(P, Q)=$ $\frac{1}{2} \sum_{\rho \in \Omega}|P(\rho)-Q(\rho)|$. 
Proof. The property follows from the fact that via CPTP maps one can go back and forth between any EHS representations of the ensembles $P(\rho)$ and $Q(\rho), \rho \in \Omega$, and the states $\bar{\rho}_{P}$ and $\bar{\rho}_{Q}$.

\section{Properties of the EHS fidelity}

The properties of the EHS fidelity 86 can be proven analogously to the properties of the EHS distance, which is why we present them without proof.

Property 1 (Positivity and normalization).

$$
\begin{gathered}
0 \leq F^{\mathrm{EHS}}(P, Q) \leq 1, \\
\forall P, Q \in \mathcal{P}_{\Omega},
\end{gathered}
$$

with

$$
F^{\mathrm{EHS}}(P, Q)=1 \text { iff } P(\rho)=Q(\rho), \quad \forall \rho \in \Omega,
$$

and

$$
F^{\mathrm{EHS}}(P, Q)=0
$$

if and only if the supports of $\mathrm{P}$ and $\mathrm{Q}$ are orthogonal sets of states.

Property 2 (Symmetry).

$$
\begin{gathered}
F^{\mathrm{EHS}}(P, Q)=F^{\mathrm{EHS}}(Q, P), \\
\forall P, Q \in \mathcal{P}_{\Omega} .
\end{gathered}
$$

Property 3 (Strong concavity).

$$
\begin{gathered}
F^{\mathrm{EHS}}\left(p P_{1}+(1-p) P_{2}, q Q_{1}+(1-q) Q_{2}\right) \\
\geq \sqrt{p q} F^{\mathrm{EHS}}\left(P_{1}, Q_{1}\right)+\sqrt{(1-q)(1-p)} F^{\mathrm{EHS}}\left(P_{2}, Q_{2}\right), \\
\forall P_{1}, P_{2}, Q_{1}, Q_{2} \in \mathcal{P}_{\Omega}, \quad \forall p, q \in[0,1] .
\end{gathered}
$$

Property 4 (Monotonicity under generalized measurements). $F^{\mathrm{EHS}}(P, Q)$ is monotonic under generalized measurements in the sense of Definition 3, $F^{\mathrm{EHS}}(P, Q) \leq F^{\mathrm{EHS}}(M(P), M(Q))$.

Corollary (Monotonicity under CPTP maps and invariance under unitary maps). $F^{\mathrm{EHS}}(P, Q)$ is monotonic under CPTP maps and invariant under unitary maps.

Property 5 (Monotonicity under averaging). Let $\bar{P}$ denote the singleton ensemble consisting of the average state of $P(\rho), \bar{\rho}_{P}=\sum_{\rho \in \Omega} P(\rho) \rho$. Then

$$
F^{\mathrm{EHS}}(P, Q) \leq F^{\mathrm{EHS}}(\bar{P}, \bar{Q}) .
$$

Corollary. If two distributions are close, their average states are also close, i.e.,

$$
\text { if } F^{\mathrm{EHS}}(P, Q) \geq 1-\varepsilon \text {, then } F\left(\bar{\rho}_{P}, \bar{\rho}_{Q}\right) \geq 1-\varepsilon \text {. }
$$

Property 6 (The EHS fidelity is lower bounded by the Kantorovich fidelity).

$$
F^{\mathrm{EHS}}(P, Q) \geq F^{K}(P, Q) \text {. }
$$

Property 7 (Stability). Let $P(\rho)$ and $Q(\rho)$ be two ensembles of states in $\Omega$ and $R\left(\sigma^{\prime}\right)$ be an ensemble of states in $\Omega^{\prime}$, where $\Omega$ and $\Omega^{\prime}$ are sets of states of two different systems. Then,

$$
F^{\mathrm{EHS}}(P \otimes R, Q \otimes R)=F^{\mathrm{EHS}}(P, Q)
$$

Property 8 (Convex optimization). The task of finding the optimal $P(\rho, \sigma)$ and $Q(\rho, \sigma)$ in Eq. 86 is a convex optimization problem.

Limiting case 1 (Two singleton ensembles). Let $P(\rho)=\delta_{\rho \tau}, \rho, \tau \in \Omega$ and $Q(\rho)=\delta_{\rho \sigma}, \rho, \sigma \in \Omega$, i.e., each of the ensembles $P$ and $Q$ consists of only a single state. Then the fidelity between the ensembles is equal to the fidelity between the respective states,

$$
F^{\mathrm{EHS}}(P, Q)=F(\tau, \sigma) \text {. }
$$

Limiting case 2 (One singleton ensemble). Unlike the Kantorovich fidelity, when the ensemble $Q(\rho)$ consists of only one state $\sigma$, i.e., $Q(\rho)=\delta_{\rho \sigma}, \rho, \sigma \in \Omega$, the EHS fidelity between $P(\rho)$ and $Q(\rho)$ is generally not equal to the average fidelity between a state drawn from the ensemble $P(\rho)$ and the state $\sigma$,

$$
F^{\mathrm{EHS}}(P, Q) \neq \sum_{\rho \in \Omega} P(\rho) F(\rho, \sigma) .
$$

Limiting case 3 (Classical distributions). If the set $\Omega$ consists of perfectly distinguishable density matrices, i.e., $F(\rho, \sigma)=\delta_{\rho \sigma}, \forall \rho, \sigma \in \Omega$, then $F^{\mathrm{EHS}}(P, Q)$ reduces to the fidelity $F\left(\bar{\rho}_{P}, \bar{\rho}_{Q}\right)$ between the density matrices $\bar{\rho}_{P}=\sum_{\rho \in \Omega} P(\rho) \rho$ and $\bar{\rho}_{Q}=\sum_{\rho \in \Omega} Q(\rho) \rho$, which is equal to the Bhattacharyya overlap between the classical probability distributions $P$ and $Q, F^{\mathrm{EHS}}(P, Q)=$ $\sum_{\rho \in \Omega} \sqrt{P(\rho) Q(\rho)}$.

Comment. Unlike the Kantorovich fidelity, here both 'classical' limits are the same.

\section{Operational interpretations of the EHS measures}

Similarly to the Kantorovich measures, we can understand the meaning of the EHS measures from an operational point of view. However, we present an interpretation in the spirit of Sec. IV.D only for the EHS distance. For the EHS fidelity, we present an interpretation of a different type, in which an ensemble of density matrices is looked upon as the output of a stochastic quantum channel with a pure-state input. 


\section{The EHS distance}

Observe that Eq. (87) can be written as

$$
\begin{aligned}
& D^{\mathrm{EHS}}(P, Q)=\min _{P(\rho, \sigma), Q(\rho, \sigma)} \sum_{\rho, \sigma \in \Omega} \frac{P(\rho, \sigma)+Q(\rho, \sigma)}{2} \times \\
& \left\|\frac{P(\rho, \sigma)}{P(\rho, \sigma)+Q(\rho, \sigma)} \rho-\frac{Q(\rho, \sigma)}{P(\rho, \sigma)+Q(\rho, \sigma)} \sigma\right\| .
\end{aligned}
$$

It is not difficult to see that

$$
\begin{gathered}
\left\|\frac{P(\rho, \sigma)}{P(\rho, \sigma)+Q(\rho, \sigma)} \rho-\frac{Q(\rho, \sigma)}{P(\rho, \sigma)+Q(\rho, \sigma)} \sigma\right\| \\
=2 p_{\max }(\rho, \sigma)-1,
\end{gathered}
$$

where $p_{\max }(\sigma, \rho)$ is the maximum average probability with which the two states $\sigma$ and $\rho$, each occurring with prior probability $\frac{P(\rho, \sigma)}{P(\rho, \sigma)+Q(\rho, \sigma)}$ and $\frac{Q(\rho, \sigma)}{P(\rho, \sigma)+Q(\rho, \sigma)}$, respectively, can be distinguished by a measurement [ 6$]$. In the case when each of the states $\rho$ and $\sigma$ is equally likely, the quantity (132) reduces to $\frac{1}{2}\|\rho-\sigma\|$.

Imagine that Alice is given two ensembles $P(\rho)$ and $Q(\rho), \rho \in \Omega$, which are also known to Bob. With probability $1 / 2$, she chooses one of the two ensembles and draws a random state from it. Let us say that she draws the state $\rho$ from the first ensemble. She then sends this state to Bob but tells him that she is sending either the state $\rho$ drawn from the first ensemble or the state $\sigma$ drawn from the second ensemble, where Alice can choose to say a particular $\sigma$ depending on the $\rho$ she actually got. Bob's task is to distinguish from which ensemble the state he receives has been drawn, and the figure of merit of his success is the average number of times he guesses correctly. Alice's goal is to make Bob's task as difficult as possible, with the caveat that, although she is free to choose her strategy, she has to reveal it to Bob. Alice's strategy is described by the probabilities $T_{1}(\rho \mid \sigma)$ with which, when having drawn state $\rho$ from the first ensemble, she will tell Bob that the state is either $\rho$ from the first ensemble or $\sigma$ from the second ensemble, and the probabilities $T_{2}(\rho \mid \sigma)$ with which, when having drawn state $\sigma$ from the second ensemble, she will say that the sate is either $\sigma$ from the second ensemble or $\rho$ from the first ensemble. In other words, Bob is aware of the joint probabilities $P(\rho, \sigma)=P(\rho) T_{1}(\rho \mid \sigma)$ and $Q(\rho, \sigma)=T_{2}(\rho \mid \sigma) Q(\sigma)$. Obviously, the probability that Bob will be told that the state he receives is either $\rho$ from the first ensemble or $\sigma$ from the second ensemble is equal to $\frac{P(\rho, \sigma)+Q(\rho, \sigma)}{2}$, and the prior probability that in such a case the state is $\rho$ is $\frac{P(\rho, \sigma)}{P(\rho, \sigma)+Q(\rho, \sigma)}$, while the prior probability that the state is $\sigma$ is $\frac{Q(\rho, \sigma)}{P(\rho, \sigma)+Q(\rho, \sigma)}$. Then assuming that Bob performs the optimal measurement to distinguish these states with these prior probabilities, the optimal strategy for Alice is to choose $T_{1}(\rho \mid \sigma)$ and $T_{2}(\rho \mid \sigma)$ (or equivalently, $P(\rho, \sigma)$ and $Q(\rho, \sigma))$ that minimize the quantity (131). The EHS distance can then be understood as

$$
D^{\mathrm{EHS}}(P, Q)=2 p_{\max }^{\mathrm{Bob}}(P, Q)-1,
$$

where $p_{\max }^{\mathrm{Bob}}(P, Q)$ is Bob's maximal probability of success when Alice chooses her strategy optimally.

\section{The EHS fidelity}

For the EHS fidelity, we propose an interpretation which is similar to the one proposed for the square root fidelity in Ref. [38],

$$
F(\rho, \sigma)=\max |\langle\psi \mid \phi\rangle|,
$$

where maximization is taken over all pure states $|\psi\rangle$ and $|\phi\rangle$ such that $\rho=\mathcal{E}(|\psi\rangle\langle\psi|)$ and $\sigma=\mathcal{E}(|\phi\rangle\langle\phi|)$ for some CPTP map $\mathcal{E}$. According to this interpretation, if $\rho$ and $\sigma$ are the outputs of a deterministic quantum channel with pure-state inputs, the square root fidelity is an upper bound on the overlap between the input states. It turns out that the EHS fidelity provides a generalization of this idea to stochastic quantum channels.

When a generalized measurement $\mathbf{M}$ with measurement superoperators $\left\{\mathcal{M}_{i}\right\}$ is applied to a given state $\sigma$, it gives rise to an ensemble $P(\rho), \rho \in \Omega$, with $P(\rho)=\sum_{i: \sigma_{i}=\rho} p_{i}$, where $p_{i}=\operatorname{Tr}\left(\mathcal{M}_{i}(\sigma)\right)$ are the probabilities for the different measurement outcomes, and $\sigma_{i}=\mathcal{M}_{i}(\sigma) / p_{i}$ are their corresponding output states. In other words, $\mathbf{M}$ can be viewed as a stochastic quantum channel which for a given input state outputs an ensemble of states. We will use the short-cut notation $\mathbf{M}(\sigma)$ to denote the ensemble of states resulting from the action of the channel $\mathbf{M}$ on the state $\sigma$.

Theorem 3 (Channel-based interpretation of the EHS fidelity). Let $P(\rho)$ and $Q(\rho), \rho \in \Omega$, be two ensembles of density matrices on $\mathcal{H}^{S}$. Then,

$$
F^{\mathrm{EHS}}(P, Q)=\max |\langle\psi \mid \phi\rangle|,
$$

where maximization is taken over all pure states $|\psi\rangle \in$ $\mathcal{H}^{S}$ and $|\phi\rangle \in \mathcal{H}^{S}$ such that $\mathbf{M}(|\psi\rangle\langle\psi|)=\{(P(\rho), \rho)\}$, $\rho \in \Omega$, and $\mathbf{M}(|\phi\rangle\langle\phi|)=\{(Q(\rho), \rho)\}, \rho \in \Omega$, for some stochastic channel $\mathbf{M}$.

Proof. From the monotonicity of the EHS fidelity under generalized measurements it follows that for any generalized measurement $\mathbf{M}$ and two states $|\psi\rangle$ and $|\phi\rangle$,

$$
F^{\mathrm{EHS}}(\mathbf{M}(|\psi\rangle\langle\psi|), \mathbf{M}(|\phi\rangle\langle\phi|)) \geq|\langle\psi \mid \phi\rangle| .
$$

Therefore, we only have to show that there exist states $|\psi\rangle,|\phi\rangle \in \mathcal{H}^{S}$ and a generalized measurement $\mathbf{M}$, for which equality is attained.

Let $P(\rho, \sigma)$ and $Q(\rho, \sigma)$ be two joint probability distributions which achieve the maximum in Eq. (88) for the pair of probability distribution $P(\rho)$ and $Q(\rho)$. From Uhlmann's theorem 1] we know that for any pair $(\rho, \sigma) \in$ $\Omega \times \Omega$, there exist purifications $\left|\psi_{\rho, \sigma}\right\rangle^{S B} \in \mathcal{H}^{S} \otimes \mathcal{H}^{B}$ and $\left|\phi_{\rho, \sigma}\right\rangle^{S B} \in \mathcal{H}^{S} \otimes \mathcal{H}^{B}$ of $\rho$ and $\sigma$, respectively, such that $F(\rho, \sigma)=\left\langle\psi_{\rho, \sigma} \mid \phi_{\rho, \sigma}\right\rangle^{S B}$. The second system $B$ can be chosen to have the same dimension as that of $S$. Let us introduce a third system with a Hilbert space $\mathcal{H}^{E}$ of 
dimension $N^{2}$, where $N$ is the cardinality of the set $\Omega$. Let $\left\{|(\rho, \sigma)\rangle^{E}\right\},(\rho, \sigma) \in \Omega \times \Omega$, be an orthonormal basis of $\mathcal{H}^{E}$. From Eq. (88) one can readily see that the pure states

$$
\begin{aligned}
|P\rangle^{S B E} & =\sum_{\rho, \sigma \in \Omega} \sqrt{P(\rho, \sigma)}\left|\psi_{\rho, \sigma}\right\rangle^{S B}|(\rho, \sigma)\rangle^{E} \\
|Q\rangle^{S B E} & =\sum_{\rho, \sigma \in \Omega} \sqrt{Q(\rho, \sigma)}\left|\phi_{\rho, \sigma}\right\rangle^{S B}|(\rho, \sigma)\rangle^{E}
\end{aligned}
$$

by construction satisfy

$$
\langle P \mid Q\rangle^{S B E}=F^{\mathrm{EHS}}(P, Q) .
$$

Notice that there exists a unitary transformation $U \in$ $\mathcal{B}\left(\mathcal{H}^{S} \otimes \mathcal{H}^{B} \otimes \mathcal{H}^{E}\right)$ such that

$$
\begin{aligned}
& U|\psi\rangle^{S}|0\rangle^{B E}=|P\rangle^{S B E}, \\
& U|\phi\rangle^{S}|0\rangle^{B E}=|Q\rangle^{S B E},
\end{aligned}
$$

where $|0\rangle^{B E}$ is some state in $\mathcal{H}^{B} \otimes \mathcal{H}^{E}$, and $|\psi\rangle^{S}$ and $|\phi\rangle^{S}$ are states in $\mathcal{H}^{S}$. Since unitary operations preserve the overlap between states,

$$
\langle\psi \mid \phi\rangle^{S}=\langle P \mid Q\rangle^{S B E}=F^{\mathrm{EHS}}(P, Q) .
$$

But from the states $|P\rangle^{S B E}$ and $|Q\rangle^{S B E}$ we can obtain the ensembles $\{(P(\rho), \rho)\}$ and $\{(Q(\rho), \rho)\}$, respectively, by performing a destructive measurement on subsystem $\mathcal{H}^{E}$ in the basis $\left\{|(\rho, \sigma)\rangle^{E}\right\}$ and tracing out subsystem $\mathcal{H}^{B}$. Therefore, starting from the two states $|\psi\rangle^{S}$ and $|\phi\rangle^{S}$ we can obtain the ensembles $\{(P(\rho), \rho)\}$ and $\{(Q(\rho), \rho)\}$ by appending the state $|0\rangle^{B E}$, applying the unitary operation $U$, measuring in the basis $\left\{|(\rho, \sigma)\rangle^{E}\right\}$ and discarding system $B$. This operation is equivalent to a generalized measurement $\mathbf{M}$ on system $S$. This completes the proof.

\section{AN ENSEMBLE-BASED INTERPRETATION OF THE SQUARE ROOT FIDELITY}

As we pointed out in Sec. V, the EHS fidelity can be formulated without reference to an extended Hilbert space (Eq. (88)). In the case when the set $\Omega$ consists of pure states, the quantity (88) can be written as

$F^{\mathrm{EHS}}(P, Q)=\max _{P(\psi, \phi), Q(\psi, \phi)} \sum_{\psi, \phi \in \Omega} \sqrt{P(\psi, \phi) Q(\psi, \phi)}|\langle\psi \mid \phi\rangle|$,

where optimization is taken over all joint distributions $P(\psi, \phi)$ with left marginal $P(\psi)$, and $Q(\psi, \phi)$ with right marginal $Q(\phi)$. Notice that for fixed $P(\psi, \phi)$ and $Q(\psi, \phi)$, the quantity $\sum_{\psi, \phi \in \Omega} \sqrt{P(\psi, \phi) Q(\psi, \phi)}|\langle\psi \mid \phi\rangle|$ can be thought of as a generalization of the Bhattacharyya overlap between classical probability distributions over the variable $(\psi, \phi)$, where the overlap
$\sqrt{P(\psi, \phi) Q(\psi, \phi)}$ between the probabilities $P(\psi, \phi)$ and $Q(\psi, \phi)$ is modified by the factor $|\langle\psi \mid \phi\rangle|$. Heuristically, we could think that the probabilities of the two distributions are of a quantum nature, i.e., instead of $P(\psi, \phi)$ and $Q(\psi, \phi)$ at a given point $(\psi, \phi)$, we have $P(\psi, \phi)|\psi\rangle\langle\psi|$ and $Q(\psi, \phi)|\phi\rangle\langle\phi|$, whose overlap is given by $\sqrt{P(\psi, \phi) Q(\psi, \phi)}|\langle\psi \mid \phi\rangle|$. Note that expression (143) is formulated without any reference to mixed-state fidelity.

Theorem 4. The square root fidelity $F(\rho, \sigma)=$ $\operatorname{Tr} \sqrt{\sqrt{\sigma} \rho \sqrt{\sigma}}$ is equal to the maximum of the fidelity (143) between all possible pure-state ensembles whose average density matrices are equal to $\rho$ and $\sigma$, i.e.,

$$
F(\rho, \sigma)=\max _{P, Q} F^{\mathrm{EHS}}(P, Q),
$$

where maximization is taken over all $P=$ $\{(P(\psi),|\psi\rangle\langle\psi|)\}$ and $Q=\{(Q(\phi),|\phi\rangle\langle\phi|)\}$, such that

$$
\begin{aligned}
& \sum_{\psi} P(\psi)|\psi\rangle\langle\psi|=\rho, \\
& \sum_{\phi} Q(\phi)|\phi\rangle\langle\phi|=\sigma .
\end{aligned}
$$

More succinctly,

$$
F(\rho, \sigma)=\max _{P(\psi, \phi), Q(\psi, \phi),} \sum_{\gamma, \phi \in \Omega} \sqrt{P(\psi, \phi) Q(\psi, \phi)}|\langle\psi \mid \phi\rangle|,
$$

where maximization is taken over all sets of pure states $\Omega$ and joint distributions $P(\psi, \phi)$ and $Q(\psi, \phi), \psi, \phi \in \Omega$, such that

$$
\begin{aligned}
& \sum_{\psi, \phi \in \Omega} P(\psi, \phi)|\psi\rangle\langle\psi|=\rho, \\
& \sum_{\psi, \phi \in \Omega} Q(\psi, \phi)|\phi\rangle\langle\phi|=\sigma .
\end{aligned}
$$

Proof. From the monotonicity of $F^{\mathrm{EHS}}(P, Q)$ under averaging, it follows that

$$
F(\rho, \sigma) \geq \max _{P(\psi, \phi), Q(\psi, \phi),} \sum_{\psi, \phi \in \Omega} \sqrt{P(\psi, \phi) Q(\psi, \phi)}|\langle\psi \mid \phi\rangle| .
$$

To prove that there are pure-state ensembles for which equality is achieved, we will make use of Uhlmann's theorem [1] according to which

$$
F(\rho, \sigma)=\max _{|\widetilde{\psi}\rangle,|\widetilde{\phi}\rangle}|\langle\widetilde{\psi} \mid \widetilde{\phi}\rangle|,
$$

where maximization is taken over all possible purifications $|\widetilde{\psi}\rangle$ and $|\widetilde{\phi}\rangle$ of $\rho$ and $\sigma$, respectively. Let $\left|\widetilde{\psi}_{0}\right\rangle$ and $\left|\widetilde{\phi}_{0}\right\rangle$ be two purifications for which the maximum in Eq. (151) is attained. Choose an orthonormal basis 
$\{|i\rangle\}$ in the auxiliary system needed for the purification. We can write

$$
\begin{aligned}
& \left|\widetilde{\psi}_{0}\right\rangle=\sum_{i} \alpha_{i}\left|\psi_{i}\right\rangle|i\rangle, \\
& \left|\widetilde{\phi}_{0}\right\rangle=\sum_{i} \beta_{i}\left|\phi_{i}\right\rangle|i\rangle .
\end{aligned}
$$

The overlap between these states can be written as

$$
\left|\left\langle\widetilde{\psi}_{0} \mid \widetilde{\phi}_{0}\right\rangle\right|=\left|\sum_{i} \alpha_{i}^{*} \beta_{i}\left\langle\psi_{i} \mid \phi_{i}\right\rangle\right| \leq \sum_{i}\left|\alpha_{i}^{*} \beta_{i}\left\langle\psi_{i} \mid \phi_{i}\right\rangle\right| .
$$

Notice that if we change arbitrarily the phases of $\alpha_{i}$ and $\beta_{i}$ in Eqs. (152) and (153), we obtain valid (although not necessarily optimal) purifications of $\rho$ and $\sigma$. If we choose the phases such that each of the quantities $\alpha_{i}^{*} \beta_{i}\left\langle\psi_{i} \mid \phi_{i}\right\rangle$ have the same phase, then equality in Eq. (154) is attained. Therefore, for optimal purifications we have

$$
\left|\left\langle\widetilde{\psi}_{0} \mid \widetilde{\phi}_{0}\right\rangle\right|=\sum_{i}\left|\alpha_{i}\right|\left|\beta_{i}\right|\left|\left\langle\psi_{i} \mid \phi_{i}\right\rangle\right|
$$

Notice that the ensembles $\left\{\left|\alpha_{i}\right|^{2},\left|\psi_{i}\right\rangle\left\langle\psi_{i}\right|\right\}$ and $\left\{\left|\beta_{i}\right|^{2},\left|\phi_{i}\right\rangle\left\langle\phi_{i}\right|\right\}$ are such that their averages give rise to $\rho$ and $\sigma$, i.e., they are among those ensembles over which maximization in Eq. (144) is taken. But $\sum_{i}\left|\alpha_{i}\right|\left|\beta_{i}\right|\left|\left\langle\psi_{i} \mid \phi_{i}\right\rangle\right|$ is exactly of the form on the righthand side of Eq. (147), i.e., equality in Eq. (150) is attained by $\left\{\left|\alpha_{i}\right|^{2},\left|\psi_{i}\right\rangle\left\langle\psi_{i}\right|\right\}$ and $\left\{\left|\beta_{i}\right|^{2},\left|\phi_{i}\right\rangle\left\langle\phi_{i}\right|\right\}$. This completes the proof.

Clearly, all interpretations of the fidelity must be equivalent, but they provide different intuitive ways of understanding the same quantity. Theorem 4 gives an interpretation based on the pure-state ensembles from which a mixed state can be prepared by averaging and thus reflects the common intuition of mixed states as describing mixtures of pure states.

\section{DISTANCE AND FIDELITY BETWEEN STOCHASTIC QUANTUM OPERATIONS}

In practice, it often makes sense to ask how close two quantum processes are. For example, we may want to compare an ideal quantum operation which we would like to implement, with an imperfect operation that we are able to implement. Distance measures between deterministic quantum operations (CPTP maps) have been defined, e.g., in Ref. [20]. However, a similar treatment for stochastic quantum operations (generalized measurements) has been missing. Stochastic operations are an important tool for quantum information processing with applications in various areas, such as quantum control, state estimation, entanglement manipulation, and error correction, to name a few. Identifying such measures could thus be very useful.
Before we propose distinguishability measures between stochastic quantum operations, let us discuss what we mean when we say that two such operations are different. For the purposes of the present paper, we will identify a stochastic quantum operation $\mathbf{M}$ (or a generalized measurement) with an ensemble $\left\{\left(m_{i}, \overline{\mathcal{M}}_{i}\right)\right\}, m_{i} \geq 0$, of different completely positive measurement superoperators $\overline{\mathcal{M}}_{i}(\cdot)=\sum_{j} \bar{M}_{i j}(\cdot) \bar{M}_{i j}^{\dagger}$ which are normalized as

$$
\operatorname{Tr}\left(\sum_{j} \bar{M}_{i j}^{\dagger} \bar{M}_{i j}\right)=d, \forall i
$$

and satisfy

$$
\sum_{i, j} m_{i} \bar{M}_{i j}^{\dagger} \bar{M}_{i j}=I
$$

The unnormalized measurement superoperators $\mathcal{M}_{i}$ which appear in the usual description of a measurement (Eq. (3) ) are related to the normalized ones via

$$
\begin{gathered}
\overline{\mathcal{M}}_{i}=\mathcal{M}_{i} / m_{i}, \\
m_{i}=\operatorname{Tr}\left(\sum_{j} M_{i j}^{\dagger} M_{i j}\right) / d .
\end{gathered}
$$

Notice that the weights $m_{i}$ satisfy $\sum_{i} m_{i}=1$, i.e., they can be thought of as 'probabilities' and $\left\{\left(m_{i}, \overline{\mathcal{M}}_{i}\right)\right\}$ can be thought of as a 'probabilistic' ensemble of normalized superoperators $\overline{\mathcal{M}}_{i}$. Note, however, that $m_{i}$ are not equal to the probabilities of the measurement outcomes which generally depend on the input state $\rho$ and are given by $p_{i}=m_{i} \operatorname{Tr}\left(\overline{\mathcal{M}}_{i}(\rho)\right)$.

The reason why we associate different outcomes with normalized superoperators is that we want our description to explicitly emphasize the fact that measurement outcomes whose measurement superoperators differ from each other only by a factor are not considered different. This is because for us a generalized measurement is not a characterization of a particular physical device (which could produce classical readings not necessarily related to the quantum system of interest), but the most abstract characterization of an operation on the state of the quantum system, which includes information extraction as well as state transformation. Clearly, two measurement superoperators which differ from each other by a factor do not provide any different information about the state of the system prior to the measurement (according to Bayes's rule) nor give rise to different post-measurement states. Note that when we say that two normalized measurement superoperators $\overline{\mathcal{M}}_{i}(\cdot)=\sum_{j} \bar{M}_{i j}(\cdot) \bar{M}_{i j}^{\dagger}$ and $\overline{\mathcal{N}}_{k}(\cdot)=\sum_{k} \bar{N}_{k l}(\cdot) \bar{N}_{k l}^{\dagger}$ are the same, we compare them as completely positive maps, i.e., irrespectively of their operator-sum representations. In other words, $\overline{\mathcal{M}}_{i}=\overline{\mathcal{N}}_{k}$ if and only if there exists a unitary matrix with components $U_{j l}$, such that $\bar{M}_{i j}=\sum_{l} U_{j l} \bar{N}_{k l}, \forall j$ [2]. In that sense, if two measurements are described by identical ensembles of normalized measurement superoperators, they are the same measurement. Conversely, if two measurements are described by different ensembles of normalized 
measurement superoperators, they should be considered different because they either give rise to different output ensembles for some input, or provide different information about the input state, or both. Therefore, we will specify a generalized measurement $\mathbf{M}$ by the correspondence

$$
\mathbf{M} \leftrightarrow\left\{\left(m_{i}, \overline{\mathcal{M}}_{i}\right)\right\}
$$

There are many possible ways in which one can define distance between two quantum operations. The following desirable properties for a distance $D$ between deterministic quantum operations $\mathcal{E}$ and $\mathcal{F}$ were pointed out and discussed in Ref. [20]: (1) metric - the measure should be positive, symmetric, satisfy the triangle inequality, and vanish if and only if the two operations are identical; (2) computability - it should be possible to evaluate $D$ in a direct manner; (3) measurability - there should be an achievable experimental procedure for determining $D$; (4) physical interpretation - the distance should have a well motivated physical interpretation; (5) stability $D(\mathcal{I} \otimes \mathcal{E}, \mathcal{I} \otimes \mathcal{F})=D(\mathcal{E}, \mathcal{F})$, which means that unrelated physical systems should not affect the value of $D$; (6) chaining- $D\left(\mathcal{E}_{2} \otimes \mathcal{E}_{1}, \mathcal{F}_{2} \otimes \mathcal{F}_{1}\right) \leq D\left(\mathcal{E}_{1}, \mathcal{F}_{1}\right)+D\left(\mathcal{E}_{2}, \mathcal{F}_{2}\right)$, i.e., for a process composed of several steps, the total error should be less than the sum of the errors in the individual steps. We will consider the same requirements for a distance between stochastic quantum operations. In the deterministic case, in view of the above desiderata, two main approaches to distinguishing quantum operations stand out - comparison based on the Jamiołkowski isomorphism and worst-case comparison. We will adopt the same approaches here.

Since many of the properties for the following measures and their proofs are similar to those discussed in Ref. [20], we will only comment on them briefly. In what follows, we will use $D$ and $F$ to denote distance and fidelity between ensembles, which can be either of the Kantorovich or of the EHS type. We will use $\mathbf{M}(\rho)$ to denote the ensemble of output states that results from the action of a stochastic quantum operation $\mathbf{M}$ on an input state $\rho$.

\section{A. Measures based on the Jamiołkowski isomorphism}

The Jamiołkowski isomorphism [27] is a one-to-one correspondence between completely positive maps (superoperators) $\mathcal{M}: \mathcal{B}\left(\mathcal{H}^{S}\right) \rightarrow \mathcal{B}\left(\mathcal{H}^{S}\right)$ and positive operators $\rho_{\mathcal{M}} \in \mathcal{B}\left(\mathcal{H}^{A} \otimes \mathcal{H}^{S}\right)$, where $\operatorname{dim}\left(\mathcal{H}^{A}\right)=\operatorname{dim}\left(\mathcal{H}^{S}\right)=d$. The correspondence is established via

$$
\rho_{\mathcal{M}}=\mathcal{I}^{A} \otimes \mathcal{M}^{S}\left(|\Phi\rangle\left\langle\left.\Phi\right|^{A S}\right),\right.
$$

where $|\Phi\rangle^{A S}=\sum_{j}|j\rangle^{A}|j\rangle^{S} / \sqrt{d}$ is a maximally entangled state on $\mathcal{H}^{A} \otimes \mathcal{H}^{S}$ (here $\left\{|j\rangle^{A}\right\}$ and $\left\{|j\rangle^{S}\right\}$ are orthonormal bases of $\mathcal{H}^{A}$ and $\mathcal{H}^{S}$, respectively). Notice that if the completely positive map $\mathcal{M}$ is trace-preserving, the corresponding positive operator $\rho_{\mathcal{M}}$ is a density matrix, i.e, $\operatorname{Tr}\left(\rho_{\mathcal{M}}\right)=1$. However, not all density matrices on $\mathcal{H}^{A} \otimes \mathcal{H}^{S}$ correspond to CPTP maps, but only those whose reduced density matrix on subsystem $A$ is the maximally mixed state $I / d$. It is easy to see that most generally, a density matrix on $\mathcal{H}^{A} \otimes \mathcal{H}^{S}$ corresponds to a completely positive superoperator $\overline{\mathcal{M}}(\cdot)=\sum_{i} \bar{M}_{i}(\cdot) \bar{M}_{i}^{\dagger}$, which is normalized as

$$
\operatorname{Tr}\left(\sum_{i} \bar{M}_{i}^{\dagger} \bar{M}_{i}\right)=d .
$$

The reverse is also true - every completely positive superoperator on $\mathcal{B}\left(\mathcal{H}^{S}\right)$, which satisfies Eq. (162), gives rise to a density matrix when applied to $|\Phi\rangle\left\langle\left.\Phi\right|^{A S}\right.$. We therefore see that there is an isomorphism

$$
\left\{\left(m_{i}, \overline{\mathcal{M}}_{i}\right)\right\} \leftrightarrow\left\{\left(m_{i}, \rho_{\overline{\mathcal{M}}_{i}}\right)\right\}
$$

between ensembles of normalized completely positive superoperators and ensembles of density matrices. Of course, just like not every completely positive map corresponding to a density matrix is trace preserving, not every ensemble $\left\{\left(m_{i}, \mathcal{M}_{i}\right)\right\}, \sum_{i} m_{i}=1$, forms a generalized measurement $\left(\sum_{i, j} m_{i} \bar{M}_{i j}^{\dagger} \bar{M}_{i j}=I\right)$. But since the reverse is true, we can use the isomorphism to define distance and fidelity between generalized measurements through the distance and fidelity between ensembles of states.

Definition 7 (Distance between generalized measurements based on the Jamiołkowski isomorphism). Let $\mathbf{M}$ and $\mathbf{N}$ be two generalized measurements acting on $\mathcal{B}\left(\mathcal{H}^{S}\right)$. Then,

$$
\begin{gathered}
D_{\text {iso }}(\mathbf{M}, \mathbf{N}) \equiv \\
D\left(\mathcal { I } ^ { A } \otimes \mathbf { M } ^ { S } \left(|\Phi\rangle\left\langle\left.\Phi\right|^{A S}\right), \mathcal{I}^{A} \otimes \mathbf{N}^{S}\left(|\Phi\rangle\left\langle\left.\Phi\right|^{A S}\right)\right),\right.\right.
\end{gathered}
$$

where $\mathcal{I}^{A} \otimes \mathbf{M}^{S}$ and $\mathcal{I}^{A} \otimes \mathbf{N}^{S}$ denote the generalized measurements $\mathbf{M}$ and $\mathbf{N}$ applied locally on subsystem $S$ and $|\Phi\rangle^{A S}=\sum_{j}|j\rangle^{A}|j\rangle^{S} / \sqrt{d}$ is a maximally entangled state on $\mathcal{H}^{A} \otimes \mathcal{H}^{S}$.

Property 1 (Metric). It follows from the metric properties of $D$.

Property 2 (Computability). It follows from the computability of $D$ which is either a linear program (in the Kantorovich case) or a convex-optimization problem (in the EHS case).

Property 3 (Measurability). As in the deterministic case, $D_{\text {iso }}$ can be determined by doing full process tomography [41, 42].

Property 4 (Physical interpretation). In addition to the obvious meaning of $D_{\text {iso }}$ following from its definition, it was pointed out in Ref. [20] that in the deterministic case, $D_{\text {iso }}(\mathcal{E}, \mathcal{F}) \geq \frac{1}{d} \sum_{x} \Delta(\mathcal{E}(|x\rangle\langle x|), \mathcal{F}(|x\rangle\langle x|))$, where the sum is over a set of orthonormal basis states $|x\rangle$ which can be thought of as the different instances of a computational problem. In a similar manner, it can be seen that $D_{\text {iso }}(\mathbf{M}, \mathbf{N}) \geq \frac{1}{d} \sum_{x} \Delta(\mathbf{M}(|x\rangle\langle x|), \mathbf{N}(|x\rangle\langle x|))$.

Property 5 (Stability). It follows from the stability of $D$. 
Property 6 (Chaining). The proof of this property assumes monotonicity of $D$ under generalized measurements and therefore it holds for the EHS distance. Similarly to the deterministic case [20], it can be shown that $D_{\text {iso }}$ satisfies $D_{\text {iso }}\left(\mathbf{M}_{2} \circ \mathbf{M}_{1}, \mathbf{N}_{2} \circ \mathbf{N}_{1}\right) \leq D_{\text {iso }}\left(\mathbf{M}_{2}, \mathbf{N}_{2}\right)+$ $D_{\text {iso }}\left(\mathbf{M}_{1}, \mathbf{N}_{1}\right)$, provided that $\mathbf{N}_{1}$ is a unital measurement, i.e., $\sum_{j} n_{1 j} \mathcal{N}_{1 j}(I)=I$, where $\left\{\left(n_{1 j}, \overline{\mathcal{N}}_{1 j}\right)\right\}$ is the ensemble of normalized measurement superoperators corresponding to $\mathbf{N}_{1}$.

Definition 8 (Fidelity between generalized measurements based on the Jamiołkowski isomorphism). Let $\mathbf{M}$ and $\mathbf{N}$ be two generalized measurements acting on $\mathcal{B}\left(\mathcal{H}^{S}\right)$. Then,

$$
\begin{gathered}
F_{\text {iso }}(\mathbf{M}, \mathbf{N}) \equiv \\
F\left(\mathcal { I } ^ { A } \otimes \mathbf { M } ^ { S } \left(|\Phi\rangle\left\langle\left.\Phi\right|^{A S}\right), \mathcal{I}^{A} \otimes \mathbf{N}^{S}\left(|\Phi\rangle\left\langle\left.\Phi\right|^{A S}\right)\right) .\right.\right.
\end{gathered}
$$

The fidelity satisfies similar properties to those of the distance, except for the triangle inequality.

\section{B. Measures based on worst-case comparison}

Definition 9 (Distance between generalized measurements based on the worst case). Let $\mathbf{M}$ and $\mathbf{N}$ be two generalized measurements acting on $\mathcal{B}\left(\mathcal{H}^{S}\right), \operatorname{dim}\left(\mathcal{H}^{S}\right)=d$. Introduce an ancillary system $A$ with a Hilbert space $\mathcal{H}^{A}, \operatorname{dim}\left(\mathcal{H}^{A}\right)=d$. Then,

$$
\begin{gathered}
D_{\max }(\mathbf{M}, \mathbf{N}) \equiv \\
\max _{|\psi\rangle} D\left(\mathcal{I}^{A} \otimes \mathbf{M}^{S}(|\psi\rangle\langle\psi|), \mathcal{I}^{A} \otimes \mathbf{N}^{S}(|\psi\rangle\langle\psi|)\right),
\end{gathered}
$$

where maximum is taken over all $|\psi\rangle \in \mathcal{H}^{A} \otimes \mathcal{H}^{S}$.

The definition is based on a maximization over states in an extended Hilbert space in order to guarantee stability of the distance, as it is known that without this extension even the analogously defined distance between CPTP maps based on the trace distance is not stable [39]. Note that this definition takes maximum over pure-state inputs. As we saw in Sec. IV.E, a generalized measurement can be defined to act on ensembles of mixed states so that it most generally transforms ensembles of density matrices into ensembles of density matrices. However, it is easy to see that one cannot obtain a larger value by maximizing over mixed states or ensembles of mixed states. This follows from the joint convexity of $D$ with respect to ensembles and from the joint convexity of $\Delta$ with respect to mixed states.

Property 1 (Metric). It follows from the metric properties of $D$. (The fact that the distance between different measurements is non-zero follows from the fact that for the input state $|\Phi\rangle^{S A}$, different measurements yield different output ensembles.)

Property 2 (Computability). We already pointed out that the measure $D$ for any particular pair of ensembles is computable. In Ref. [20] it was argued that in the case of deterministic operations, the corresponding optimization in Eq. (166) is a convex optimization problem and therefore computable. By a similar argument it can be seen that for stochastic quantum operations, finding the maximum in Eq. (166) is also a convex optimization problem.

Property 3 (Measurability). Here too, the value of $D_{\max }$ can be determined using quantum process tomography [41, 42].

Property 4 (Physical interpretation). The physical meaning of $D_{\max }$ follows directly from its definition and the physical meaning of $D$.

Property 5 (Stability). The proof goes along the same lines as the proof for the deterministic case (Ref. [20]) - all one needs to show is that the quantity (166) is independent of the dimension of system $A$, as long as this dimension is greater than or equal to $d$. This follows from the observation that an input state which achieves the maximum in Eq. (166) can have at most $d$ Schmidt coefficients, which implies that there is a subspace of $\mathcal{H}^{A}$ with dimension $d$ such that the maximum can be achieved by maximization inside that subspace.

Property 6 (Chaining). The chaining property follows from the triangle inequality and the monotonicity of $D$ under generalized measurements, i.e., it holds for the EHS distance.

Definition 10 (Fidelity between generalized measurements based on the worst case). Let $\mathbf{M}$ and $\mathbf{N}$ be two generalized measurements acting on $\mathcal{B}\left(\mathcal{H}^{S}\right), \operatorname{dim}\left(\mathcal{H}^{S}\right)=d$. Introduce an ancillary system $A$ with a Hilbert space $\mathcal{H}^{A}, \operatorname{dim}\left(\mathcal{H}^{A}\right)=d$. Then,

$$
\begin{gathered}
F_{\min }(\mathbf{M}, \mathbf{N}) \equiv \\
\min _{|\psi\rangle} F\left(\mathcal{I}^{A} \otimes \mathbf{M}^{S}(|\psi\rangle\langle\psi|), \mathcal{I}^{A} \otimes \mathbf{N}^{S}(|\psi\rangle\langle\psi|)\right),
\end{gathered}
$$

where minimum is taken over all $|\psi\rangle \in \mathcal{H}^{A} \otimes \mathcal{H}^{S}$.

The fidelity $F_{\min }$ satisfies properties analogous to those of $D_{\max }$ with the exception of the triangle inequality.

\section{Distance and fidelity between POVMs}

A very useful concept in quantum information is that of a positive operator-valued measure (POVM) - a set of positive operators $\left\{E_{i}\right\}, E_{i}>0$, which sum up to the identity, $\sum_{i} E_{i}=I$. A POVM provides the most general description of a quantum measurement in situations where one is not interested in the post-measurement state. In terms of the measurement superoperators $\mathcal{M}_{i}$, the POVM elements are given by $E_{i}=\sum_{j} M_{i j}^{\dagger} M_{i j}$, i.e., there is no unique generalized measurement which corresponds to a given POVM. Similarly to the case of generalized measurements, we can express a POVM as an ensemble of normalized POVM elements, $\left\{\left(m_{i}, \bar{E}_{i}\right)\right\}$, where $m_{i}=\operatorname{Tr}\left(E_{i}\right) / d, \bar{E}_{i}=E_{i} / m_{i}$. Notice that the operators

$$
\rho_{\bar{E}_{i}} \equiv \bar{E}_{i} / d
$$

are density matrices $\left(\operatorname{Tr}\left(\rho_{\bar{E}_{i}}\right)=1\right)$, i.e., there is a one-toone correspondence between POVMs and ensembles of 
density matrices $\left\{\left(m_{i}, \rho_{\bar{E}_{i}}\right)\right\}$ which satisfy $\sum_{i} m_{i} \rho_{\bar{E}_{i}}=$ $I / d$. Therefore, we can compare POVMs directly using the distinguishability measures between ensembles of states.

Definition 11 (Distance between POVMs). Let $\left\{E_{i}\right\}$ and $\left\{G_{j}\right\}$ be two POVMs and let $P_{E} \equiv\left\{\left(m_{i}, \rho_{\bar{E}_{i}}\right)\right\}$ $\left(m_{i}=\operatorname{Tr}\left(E_{i}\right) / d, \rho_{\bar{E}_{i}}=E_{i} /\left(m_{i} d\right)\right)$ and $P_{G} \equiv\left\{\left(n_{j}, \rho_{\bar{G}_{j}}\right)\right\}$ $\left(n_{j}=\operatorname{Tr}\left(G_{j}\right) / d, \rho_{\bar{G}_{j}}=G_{j} /\left(n_{j} d\right)\right)$ be the ensembles of density matrices that correspond to them. Then,

$$
D_{\mathrm{POVM}}\left(\left\{E_{i}\right\},\left\{G_{j}\right\}\right) \equiv D\left(P_{E}, P_{G}\right) .
$$

Definition 12 (Fidelity between POVMs). Let $\left\{E_{i}\right\}$ and $\left\{G_{j}\right\}$ be two POVMs and let $P_{E} \equiv\left\{\left(m_{i}, \rho_{\bar{E}_{i}}\right)\right\}$ $\left(m_{i}=\operatorname{Tr}\left(E_{i}\right) / d, \rho_{\bar{E}_{i}}=E_{i} /\left(m_{i} d\right)\right)$ and $P_{G} \equiv\left\{\left(n_{j}, \rho_{\bar{G}_{j}}\right)\right\}$ $\left(n_{j}=\operatorname{Tr}\left(G_{j}\right) / d, \rho_{\bar{G}_{j}}=G_{j} /\left(n_{j} d\right)\right)$ be the ensembles of density matrices that correspond to them. Then,

$$
F_{\mathrm{POVM}}\left(\left\{E_{i}\right\},\left\{G_{j}\right\}\right) \equiv F\left(P_{E}, P_{G}\right)
$$

The properties of these measures can be obtained in a straightforward manner from the properties of the distance and fidelity between states. We only remark that the ensemble of states $P_{E}=\left\{\left(m_{i}, \rho_{\bar{E}_{i}}\right)\right\}$ that corresponds to a given POVM $\left\{E_{i}\right\}$ has the following operational meaning - it is the ensemble of states of system $A$ that we obtain from the maximally entangled state $|\Phi\rangle^{A S}$ if we perform the destructive POVM $\left\{E_{i}\right\}$ on subsystem $S$,

$$
|\Phi\rangle\left\langle\left.\Phi\right|^{A S} \rightarrow \rho_{\bar{E}_{i}}^{A}=\operatorname{Tr}_{S}\left(I^{A} \otimes E_{i}^{S}|\Phi\rangle\left\langle\left.\Phi\right|^{A S}\right) / m_{i},\right.\right.
$$

with probability $m_{i}=\operatorname{Tr}\left(I^{A} \otimes E_{i}^{S}|\Phi\rangle\left\langle\left.\Phi\right|^{A S}\right)=\operatorname{Tr}\left(E_{i}^{S}\right)\right.$.

As quantum detector tomography is now within the reach of experimental technology [26], it becomes relevant to ask how much a real quantum detector differs from an ideal one. The distance and fidelity between POVMs introduced in this section provide rigorous means of quantifying such difference.

\section{CONCLUSION}

In this paper we defined measures of distance and fidelity between probabilistic ensembles of quantum states and used them to define measures of distance and fidelity between stochastic quantum operations. We proposed two types of measures between ensembles.

The first one is based on the ability of one ensemble to mimic another and leads to measures of a Kantorovich type, which appear in the context of optimal transportation and can be computed as linear programs. However, when based on the trace distance or the square root fidelity, these measures are not monotonic under generalized measurements. We derived necessary and sufficient conditions that the basic measures of distance and fidelity between states have to satisfy in order for the corresponding Kantorovich distance and fidelity to be monotonic under measurements (Theorem 2). An interesting open problem is whether measures of distance and fidelity that satisfy the conditions of Theorem 2 exist.

The second type of measures is based on the notion of an extended-Hilbert-space (EHS) representation of an ensemble. We showed that for every ensemble there is a class of valid EHS representations and defined the measures as a minimum (maximum) of the trace distance (square root fidelity) between all EHS representations of the ensembles being compared. These measures, which are monotonic under generalized measurements, can be computed as convex optimization problems. We provided operational interpretations for the measures and showed that the EHS fidelity is an upper bound of the overlap between all possible pure-state inputs that could give rise to the two ensembles being compared under the action of a stochastic quantum operation. We also used the EHS fidelity between ensembles to provide a novel interpretation of the square root fidelity between density matrices. We showed that the square root fidelity is equal to the minimum fidelity between all possible pure-state ensembles from which the density matrices being compared can be obtained.

An interesting question is whether any of the measures between ensembles that we introduced can be used to define a Riemannian metric on the space of ensembles, which endows the space with geometrical notions such as volume or geodesics. Clearly, the measures based on the trace distance would not induce a Riemannian metric because the trace distance is known not to be Riemannian [40]. The Kantorovich fidelity is not a good candidate either because in one of the classical limits it reduces to a function of the Kolmogorov distance. However, we can define an EHS distance which is a generalization of the Bures distance between density matrices, $B^{\mathrm{EHS}}(P, Q)=$ $\sqrt{1-F^{\mathrm{EHS}}(P, Q)}$, or an EHS angle which is a generalization of the Bures angle, $A^{\mathrm{EHS}}(P, Q)=\arccos F^{\mathrm{EHS}}(P, Q)$. It is known that the Bures distance and angle induce a Riemannian metric, and it would be interesting to see if their EHS generalizations induce such a metric on the space of ensembles. This problem is left open for future investigation.

Finally, based on the measures between ensembles, we defined two types of distinguishability measures between generalized measurements. The first one is based on the Jamiołkowski isomorphism and the second one on the worst-case comparison. These measures are generalizations of the distance and fidelity between CPTP maps proposed in Ref. [20] and similarly to them satisfy the desiderata outlined in Ref. [20]. One of the desired properties - the chaining property - is satisfied only by the measures based on the EHS distance and fidelity since this property requires monotonicity under generalized measurements of the corresponding measures between ensembles of states. In addition to generalized measurements, we also defined distinguishability measures between POVMs. The proposed measures may find various applications as they provide a rigorous general tool for assessing the performance of non-destructive and 
destructive measurement schemes.

\section{Appendix A: CONTINUITY OF THE AVERAGE OF A CONTINUOUS FUNCTION WITH RESPECT TO THE KANTOROVICH DISTANCE}

Let $h(\rho)$ be a bounded function which is continuous with respect to the distance $\Delta$, i.e., for every $\delta>0$, there exists $\varepsilon>0$, such that for all $\rho$ and $\sigma$ for which

$$
\Delta(\rho, \sigma) \leq \varepsilon,
$$

we have

$$
|h(\rho)-h(\sigma)| \leq \frac{1}{2} \delta .
$$

(The factor of $\frac{1}{2}$ in front of $\delta$ is chosen for convenience.) Let $\bar{h}_{P}$ denote the average of the function $h(\rho)$ over the ensemble $P(\rho), \rho \in \Omega$,

$$
\bar{h}_{P}=\sum_{\rho \in \Omega} P(\rho) h(\rho) .
$$

We will prove that for every $\delta>0$, there exists $\varepsilon^{\prime}>0$, such that for all $P, Q \in \mathcal{P}_{\Omega}$ for which

$$
D^{K}(P, Q) \leq \varepsilon^{\prime},
$$

we have

$$
\left|\bar{h}_{P}-\bar{h}_{Q}\right| \leq \delta .
$$

Assume that $D^{K}(P, Q) \leq \varepsilon^{\prime}$. Let $\Pi(\rho, \sigma)$ be a joint distribution for which the minimum in the definition (12) of $D^{K}(P, Q)$ is achieved, i.e.,

$$
D^{K}(P, Q)=\sum_{\rho, \sigma \in \Omega} \Pi(\rho, \sigma) \Delta(\rho, \sigma) \leq \varepsilon^{\prime} .
$$

Define the sets $\Omega_{>\varepsilon}$ and $\Omega_{<\varepsilon}$ as the sets of all pairs of states $(\rho, \sigma)$ for which $\Delta(\rho, \sigma)>\varepsilon$ and $\Delta(\rho, \sigma) \leq \varepsilon$, respectively. The sum in Eq. (A6) can then be split in two sums,

$$
\sum_{\Omega_{>\varepsilon}} \Pi(\rho, \sigma) \Delta(\rho, \sigma)+\sum_{\Omega_{\leq \varepsilon}} \Pi(\rho, \sigma) \Delta(\rho, \sigma) \leq \varepsilon^{\prime} .
$$

The first sum obviously can be bounded as follows,

$$
\sum_{\Omega_{>\varepsilon}} \Pi(\rho, \sigma) \varepsilon \leq \sum_{\Omega_{>\varepsilon}} \Pi(\rho, \sigma) \Delta(\rho, \sigma) \leq \varepsilon^{\prime},
$$

which implies that

$$
\sum_{\Omega_{>\varepsilon}} \Pi(\rho, \sigma) \leq \frac{\varepsilon^{\prime}}{\varepsilon} .
$$

On the other hand, we have

$$
\begin{gathered}
\left|\bar{h}_{P}-\bar{h}_{Q}\right|=\left|\sum_{\rho \in \Omega} P(\rho) h(\rho)-\sum_{\sigma \in \Omega} Q(\sigma) h(\sigma)\right| \\
=\left|\sum_{\rho, \sigma \in \Omega} \Pi(\rho, \sigma) h(\rho)-\sum_{\rho, \sigma \in \Omega} \Pi(\rho, \sigma) h(\sigma)\right| \\
\leq \sum_{\rho, \sigma \in \Omega} \Pi(\rho, \sigma)|h(\rho)-h(\sigma)|= \\
\sum_{\Omega_{>\varepsilon}} \Pi(\rho, \sigma)|h(\rho)-h(\sigma)|+\sum_{\Omega_{\leq \varepsilon}} \Pi(\rho, \sigma)|h(\rho)-h(\sigma)| .
\end{gathered}
$$

Since $h(\rho)$ is bounded, there exists a constant $h_{\max }>0$ such that $|h(\rho)-h(\sigma)| \leq h_{\max }$ for all $\rho$ and $\sigma$. Using this fact, together with Eq. (A9) and the assumption that for all $(\rho, \sigma) \in \Omega_{\leq \varepsilon},|h(\rho)-h(\sigma)| \leq \frac{1}{2} \delta$, we can upper bound the last line in Eq. (A10) as follows:

$$
\begin{gathered}
\sum_{\Omega_{>\varepsilon}} \Pi(\rho, \sigma)|h(\rho)-h(\sigma)|+\sum_{\Omega_{\leq \varepsilon}} \Pi(\rho, \sigma)|h(\rho)-h(\sigma)| \leq \\
\frac{\varepsilon^{\prime}}{\varepsilon} h_{\max }+\sum_{\Omega_{\leq \varepsilon}} \Pi(\rho, \sigma) \frac{1}{2} \delta \leq \\
\frac{\varepsilon^{\prime}}{\varepsilon} h_{\max }+\frac{1}{2} \delta .
\end{gathered}
$$

Therefore, we see that by choosing

$$
\varepsilon^{\prime} \leq \frac{\delta \varepsilon}{2 h_{\max }},
$$

we obtain

$$
\left|\bar{h}_{P}-\bar{h}_{Q}\right| \leq \delta .
$$

Since $\delta$ was arbitrarily chosen, the property follows.

\section{Appendix B: NON-MONOTONICITY UNDER GENERALIZED MEASUREMENTS OF THE KANTOROVICH MEASURES}

To show that the Kantorovich distance is not monotonic under measurements, let us look at a particular example. Consider the case of two singleton ensembles consisting of the states $\sum_{i} p_{i} \rho_{i} \otimes|i\rangle\langle i|$ and $\sum_{i} q_{i} \sigma_{i} \otimes|i\rangle\langle i|$, respectively, where the states $\{|i\rangle\}$ are an orthonormal set, $\langle i \mid j\rangle=\delta_{i j}$. Imagine that we apply a nondestructive projective measurement on the second subsystem in the basis $\{|i\rangle\}$. This measurement yields the ensembles $\left\{\left(p_{i}, \rho_{i} \otimes|i\rangle\langle i|\right)\right\}$ and $\left\{\left(q_{i}, \sigma_{i} \otimes|i\rangle\langle i|\right)\right\}$, which we will denote by $p$ and $q$ for short. Observe that the Kantorovich distance between the resulting ensembles, as defined in Eq. (12), is equal to

$$
D^{K}(p, q)=\frac{1}{2} \sum_{i}\left(\min \left(p_{i}, q_{i}\right)\left\|\rho_{i}-\sigma_{i}\right\|+\left|p_{i}-q_{i}\right|\right) .
$$


This follows from the observation that for any joint probability distribution $\Pi\left(\rho_{i} \otimes|i\rangle\left\langle i\left|, \sigma_{j} \otimes\right| j\right\rangle\langle j|\right)$, the quantity in Eq. (11) reads

$$
\begin{aligned}
D_{\Pi}(p, q)= & \frac{1}{2} \sum_{i} \Pi\left(\rho_{i} \otimes|i\rangle\left\langle i\left|, \sigma_{i} \otimes\right| i\right\rangle\langle i|\right)\left\|\rho_{i}-\sigma_{i}\right\| \\
& +\sum_{i \neq j} \Pi\left(\rho_{i} \otimes|i\rangle\left\langle i\left|, \sigma_{j} \otimes\right| j\right\rangle\langle j|\right)
\end{aligned}
$$

because $\langle i \mid j\rangle=\delta_{i j}$. Since $\sum_{i} \Pi\left(\rho_{i} \otimes|i\rangle\left\langle i\left|, \sigma_{i} \otimes\right| i\right\rangle\langle i|\right)+$ $\sum_{i \neq j} \Pi\left(\rho_{i} \otimes|i\rangle\left\langle i\left|, \sigma_{j} \otimes\right| j\right\rangle\langle j|\right)=1$, and $\left\|\rho_{i}-\sigma_{i}\right\| \leq 1$, if each of the terms $\Pi\left(\rho_{i} \otimes|i\rangle\left\langle i\left|, \sigma_{i} \otimes\right| i\right\rangle\langle i|\right)$ is equal to its maximal possible value consistent with the marginal conditions, then the value of $D_{\Pi}(p, q)$ would be minimal and it would be equal to the Kantorovich distance $D^{K}(p, q)$. The maximum possible value of $\Pi\left(\rho_{i} \otimes|i\rangle\left\langle i\left|, \sigma_{i} \otimes\right| i\right\rangle\langle i|\right)$ consistent with the marginal probability distributions is $\min \left(p_{i}, q_{i}\right)$ because if, say, $\min \left(p_{i}, q_{i}\right)=p_{i}$ and $\Pi\left(\rho_{i} \otimes\right.$ $\left.|i\rangle\left\langle i\left|, \sigma_{i} \otimes\right| i\right\rangle\langle i|\right)>p_{i}$, then $\sum_{j} \Pi\left(\rho_{i} \otimes|i\rangle\left\langle i\left|, \sigma_{j} \otimes\right| j\right\rangle\langle j|\right)$ would be strictly larger than $p_{i}$, while by definition it has to be equal to $p_{i}$. Each of these values is achievable because there exist joint probability distributions $\Pi\left(\rho_{i} \otimes|i\rangle\left\langle i\left|, \sigma_{j} \otimes\right| j\right\rangle\langle j|\right)$ with the correct marginals that satisfy

$$
\Pi\left(\rho_{i} \otimes|i\rangle\left\langle i\left|, \sigma_{i} \otimes\right| i\right\rangle\langle i|\right)=\min \left(p_{i}, q_{i}\right), \forall i .
$$

The latter can be seen from the fact that $\Pi\left(\rho_{i} \otimes|i\rangle\langle i|, \sigma_{j} \otimes\right.$ $|j\rangle\langle j|)$ describes a transportation plan which tells us what probability weights taken from $p_{i}$ and $q_{j}$ come together as we transport one distribution on top of the other. The condition $\Pi\left(\rho_{i} \otimes|i\rangle\left\langle i\left|, \sigma_{i} \otimes\right| i\right\rangle\langle i|\right)=\min \left(p_{i}, q_{i}\right)$ simply specifies how to pair certain parts of the two distributions, each having a total weight of $\sum_{i} \min \left(p_{i}, q_{i}\right)$. Since the remaining parts of the two distributions have equal weights, $1-\sum_{i} \min \left(p_{i}, q_{i}\right)$, there certainly exists a transportation plan according to which one can be mapped on top of the other. Therefore, the Kantorovich distance between $p$ and $q$ is given by Eq. (B1).

However, the Kantorovich distance between the original singleton ensembles is equal to the trace distance between the two states,

$$
\begin{gathered}
\frac{1}{2} \| \sum_{i} p_{i} \sigma_{i} \otimes|i\rangle\left\langle i\left|-\sum_{j} q_{j} \sigma_{j} \otimes\right| j\right\rangle\langle j| \| \\
=\frac{1}{2} \sum_{i}\left\|p_{i} \rho_{i}-q_{i} \sigma_{i}\right\| .
\end{gathered}
$$

Assume that for a given $i, \min \left(p_{i}, q_{i}\right)=p_{i}$. We can write

$$
\left\|p_{i} \rho_{i}-q_{i} \sigma_{i}\right\|=p_{i}\left\|\rho_{i}-\frac{q_{i}}{p_{i}} \sigma_{i}\right\| .
$$

But from the triangle inequality we have

$$
\begin{gathered}
\left\|\rho_{i}-\frac{q_{i}}{p_{i}} \sigma_{i}\right\| \leq\left\|\rho_{i}-\sigma_{i}\right\|+\left\|\sigma_{i}-\frac{q_{i}}{p_{i}} \sigma_{i}\right\| \\
=\left\|\rho_{i}-\sigma_{i}\right\|+\left(\frac{q_{i}}{p_{i}}-1\right),
\end{gathered}
$$

i.e.,

$$
\begin{gathered}
\left\|p_{i} \rho_{i}-q_{i} \sigma_{i}\right\| \leq p_{i}\left(\left\|\rho_{i}-\sigma_{i}\right\|+\left(\frac{q_{i}}{p_{i}}-1\right)\right) \\
=p_{i}\left\|\rho_{i}-\sigma_{i}\right\|+\left(q_{i}-p_{i}\right) \\
=\min \left(p_{i}, q_{i}\right)\left\|\rho_{i}-\sigma_{i}\right\|+\left|p_{i}-q_{i}\right| .
\end{gathered}
$$

Since we arbitrarily assumed which is the smaller of the two values $p_{i}$ and $q_{i}$, the inequality (B7) must hold for every $i$. Comparing Eq. (B1) and Eq. (B4), we see that

$$
\frac{1}{2} \| \sum_{i} p_{i} \sigma_{i} \otimes|i\rangle\left\langle i\left|-\sum_{j} q_{j} \sigma_{j} \otimes\right| j\right\rangle\langle j| \| \leq D^{K}(p, q) .
$$

For most choices of $\rho_{i}$ and $\sigma_{i}$, the inequality (B8 $)$ is strict since the triangle inequality used in Eq. (B6) is generally strict. Thus we see that the Kantorovich distance is not monotonically decreasing under measurements. Obviously it is not monotonically increasing either because it decreases under CPTP maps (Property 6, Sec. IV.B).

For the Kantorovich fidelity, we already observed that its values in the two classical limits are not the same: the fidelity between the two singleton distributions consisting of states of the form $\rho=\sum_{i} p_{i}|i\rangle\langle i|$ and $\sigma=\sum_{i} q_{i}|i\rangle\langle i|$, where $\{|i\rangle\}$ is an orthonormal set, is equal to

$$
F^{K}(P, Q)=F(\rho, \sigma)=\sum_{i} \sqrt{p_{i} q_{i}},
$$

whereas the fidelity between the ensembles $\left\{\left(p_{i},|i\rangle\langle i|\right)\right\}$ and $\left\{\left(q_{i},|i\rangle\langle i|\right)\right\}$ is equal to $\sum_{i} \min \left(p_{i}, q_{i}\right)$, which is strictly smaller than $F(\rho, \sigma)$ unless $p_{i}=q_{i}, \forall i$. The latter pair of ensembles are exactly the ensembles that result from a measurement in the $\{|i\rangle\}$ basis applied to the states $\rho$ and $\sigma$. Therefore, the Kantorovich fidelity can decrease under measurements. Clearly, it is not always decreasing because it increases under CPTP maps (Property 4, Sec. IV.C).

We can now see that the difference of the values of the Kantorovich fidelity in the two 'classical' limits discussed earlier can be linked to its lack of monotonicity under measurements. Obviously, through a projective measurement and averaging, we can go back and forth between these two limits. Since the Kantorovich fidelity is monotonic under averaging, if it were also monotonic under measurements, it would have to remain invariant under these operations since they are reversible. By the same token, any measure of distinguishability between ensembles, which is monotonic both under measurements and averaging of the ensembles, would have to have the same values in the two classical limits. As we saw for the case of the Kantorovich distance, however, the latter property by itself is not a guarantee for monotonicity.

\section{Appendix C: PROOF OF THEOREM 2}

From the proof of Property 7 in Sec. IV.B, it can be seen that if the distance (fidelity) between states is jointly 
convex (concave), the corresponding Kantorovich measure would be monotonic under averaging of the ensembles. The necessity of the conditions in Theorem 2 follows from the observation that if we apply a measurement on the second subsystem in the basis $\{|i\rangle\}$, we obtain the ensembles $\left\{\left(p_{i}, \rho_{i} \otimes|i\rangle\langle i|\right)\right\}$ and $\left\{\left(q_{i}, \sigma_{i} \otimes|i\rangle\langle i|\right)\right\}$, and if we follow the measurement by an averaging of the ensembles, we obtain the original states. If the Kantorovich measures are monotonic both under measurements and averaging, they must be invariant during the process. By an argument analogous to the one following Eq. (B1), it can be seen that a Kantorovich distance $D_{d}^{K}$ between ensembles of the form $\left\{\left(p_{i}, \rho_{i} \otimes|i\rangle\langle i|\right)\right\}$ and $\left\{\left(q_{i}, \sigma_{i} \otimes|i\rangle\langle i|\right)\right\}$ is equal to $\sum_{i}\left(\min \left(p_{i}, q_{i}\right) d\left(\rho_{i}, \sigma_{i}\right)+\frac{1}{2}\left|p_{i}-q_{i}\right|\right)$. Similarly, a Kantorovich fidelity $F_{f}^{K}$ between ensembles of the form $\left\{\left(p_{i}, \rho_{i} \otimes|i\rangle\langle i|\right)\right\}$ and $\left\{\left(q_{i}, \sigma_{i} \otimes|i\rangle\langle i|\right)\right\}$ is equal to $\sum_{i} \min \left(p_{i}, q_{i}\right) f\left(\rho_{i}, \sigma_{i}\right)$.

To prove the sufficiency of condition (71), consider two ensembles of states $P(\rho)$ and $Q(\sigma)$. Let $\Pi(\rho, \sigma)$ be a joint probability distribution that attains the minimum in Eq. (12), i.e.,

$$
D_{d}^{K}(P, Q)=\sum_{\rho, \sigma \in \Omega} \Pi(\rho, \sigma) d(\rho, \sigma) .
$$

According to condition (71),

$$
\begin{gathered}
D_{d}^{K}(P, Q)= \\
d\left(\sum_{\rho, \sigma \in \Omega} \Pi(\rho, \sigma) \rho \otimes|\rho \sigma\rangle\left\langle\rho \sigma\left|, \sum_{\rho, \sigma \in \Omega} \Pi(\rho, \sigma) \sigma \otimes\right| \rho \sigma\right\rangle\langle\rho \sigma|\right),
\end{gathered}
$$

where $|\rho \sigma\rangle$ is a set of orthonormal states, $\left\langle\rho \sigma \mid \rho^{\prime} \sigma^{\prime}\right\rangle=$ $\delta_{\rho \rho^{\prime}} \delta_{\sigma \sigma^{\prime}}$. Let $\left\{\mathcal{M}_{i}\right\}, \mathcal{M}_{i}(\rho)=\sum_{j} M_{i j} \rho M_{i j}^{\dagger}$, be a set of completely positive maps that form a generalized measurement, $\sum_{i, j} M_{i j}^{\dagger} M_{i j}=I$. Consider the following CPTP map:

$$
\mathcal{M}(\rho)=\sum_{i} \mathcal{M}_{i}(\rho) \otimes|i\rangle\langle i|,
$$

where $\{|i\rangle\}$ is an orthonormal set of states in the Hilbert space of some additional system (this map is not dimension-preserving). From the monotonicity of $d(\rho, \sigma)$ under CPTP maps and property (71), it follows that

$$
\begin{gathered}
D_{d}^{K}(P, Q)= \\
d\left(\sum_{\rho, \sigma \in \Omega} \Pi(\rho, \sigma) \rho \otimes|\rho \sigma\rangle\left\langle\rho \sigma\left|, \sum_{\rho, \sigma \in \Omega} \Pi(\rho, \sigma) \sigma \otimes\right| \rho \sigma\right\rangle\langle\rho \sigma|\right) \\
\geq d\left(\sum_{\rho, \sigma \in \Omega} \sum_{i} \Pi(\rho, \sigma) \mathcal{M}_{i}(\rho) \otimes|\rho \sigma\rangle\langle\rho \sigma|\otimes| i\rangle\langle i|,\right. \\
\left.\sum_{\rho, \sigma \in \Omega} \sum_{i} \Pi(\rho, \sigma) \mathcal{M}_{i}(\sigma) \otimes|\rho \sigma\rangle\langle\rho \sigma|\otimes| i\rangle\langle i|\right) \\
=\sum_{\rho, \sigma \in \Omega} \sum_{i} \min \left(\Pi(\rho, \sigma) p_{i}(\rho), \Pi(\rho, \sigma) p_{i}(\sigma)\right) d\left(\rho_{i}, \sigma_{i}\right)+ \\
\frac{1}{2} \sum_{\rho, \sigma \in \Omega} \sum_{i}\left|\Pi(\rho, \sigma) p_{i}(\rho)-\Pi(\rho, \sigma) p_{i}(\sigma)\right|,
\end{gathered}
$$

where $p_{i}(\rho)=\operatorname{Tr}\left(\mathcal{M}_{i}(\rho)\right), \rho_{i}=\mathcal{M}_{i}(\rho) / p_{i}(\rho)$. Now observe that there exists a joint probability distribution $\breve{\Pi}\left(\rho_{i}, \sigma_{j}\right)$ that satisfies

$$
\breve{\Pi}\left(\sigma_{i}, \rho_{i}\right)=\min \left(\Pi(\rho, \sigma) p_{i}(\rho), \Pi(\rho, \sigma) p_{i}(\sigma)\right)
$$

and has marginals

$$
\begin{aligned}
& \sum_{\sigma \in \Omega} \sum_{j} \breve{\Pi}\left(\rho_{i}, \sigma_{j}\right)=P(\rho) p_{i}(\rho), \\
& \sum_{\rho \in \Omega} \sum_{i} \breve{\Pi}\left(\rho_{i}, \sigma_{j}\right)=Q(\sigma) p_{j}(\sigma) .
\end{aligned}
$$

This is because condition (C5) is compatible with the marginal conditions (C6) and (C7), which follows from an argument analogous to the one in the paragraph after Eq.(B3). For this distribution, we can write

$$
\begin{gathered}
\sum_{\rho, \sigma \in \Omega} \sum_{i, j} \breve{\Pi}\left(\rho_{i}, \sigma_{j}\right) d\left(\rho_{i}, \sigma_{j}\right)= \\
\sum_{\rho, \sigma \in \Omega} \sum_{i} \min \left(\Pi(\rho, \sigma) p_{i}(\rho), \Pi(\rho, \sigma) p_{i}(\sigma)\right) d\left(\rho_{i}, \sigma_{i}\right)+ \\
\sum_{\rho, \sigma \in \Omega} \sum_{i \neq j} \breve{\Pi}\left(\rho_{i}, \sigma_{j}\right) d\left(\rho_{i}, \sigma_{j}\right)
\end{gathered}
$$

But we have that

$$
d\left(\rho_{i}, \sigma_{j}\right) \leq 1
$$

and

$$
\begin{gathered}
\sum_{\rho, \sigma \in \Omega} \sum_{i \neq j} \breve{\Pi}\left(\rho_{i}, \sigma_{j}\right)= \\
1-\sum_{\rho, \sigma \in \Omega} \sum_{i} \min \left(\Pi(\rho, \sigma) p_{i}(\rho), \Pi(\rho, \sigma) p_{i}(\sigma)\right),
\end{gathered}
$$

from which we obtain that the second sum on the righthand side of Eq. (C8) satisfies

$$
\begin{gathered}
\sum_{\rho, \sigma \in \Omega} \sum_{i \neq j} \breve{\Pi}\left(\rho_{i}, \sigma_{j}\right) d\left(\rho_{i}, \sigma_{j}\right) \leq \\
1-\sum_{\rho, \sigma \in \Omega} \sum_{i} \min \left(\Pi(\rho, \sigma) p_{i}(\rho), \Pi(\rho, \sigma) p_{i}(\sigma)\right) \\
=\frac{1}{2} \sum_{\rho, \sigma \in \Omega} \sum_{i}\left|\Pi(\rho, \sigma) p_{i}(\rho)-\Pi(\rho, \sigma) p_{i}(\sigma)\right| .
\end{gathered}
$$

Combining Eqs. (C8) and (C11), we see that the expression on the right-hand side of the last equality in Eq. (C4) is greater than or equal to

$$
\sum_{\rho, \sigma \in \Omega} \sum_{i, j} \breve{\Pi}\left(\rho_{i}, \sigma_{j}\right) d\left(\rho_{i}, \sigma_{j}\right) \equiv D_{d, \breve{\Pi}}(M(P), M(Q)) .
$$

But notice that the quantity $\mathrm{C12}$ is greater than or equal to $D_{d}^{K}(M(P), M(Q))$, where $M: \mathcal{P}_{\Omega} \rightarrow \mathcal{P}_{\Omega_{\mathrm{M}}}$ is 
the map on the original probability distributions induced by the measurement $\mathbf{M}$ with measurement superoperators $\left\{\mathcal{M}_{i}\right\}$. This is because $\breve{\Pi}\left(\rho_{i}, \sigma_{j}\right)$ is a joint probability distribution with marginals $P(\rho) p_{i}(\rho)$ and $Q(\sigma) p_{j}(\sigma)$, which are consistent with the distributions $M(P)$ and $M(Q)$ over $\Omega_{\mathbf{M}}$, and therefore the quantity Eq. (C12) is among those quantities over which the minimum in the definition of $D_{d}^{K}(M(P), M(Q))$ is taken. Therefore, we have shown that for an arbitrary generalized measurement,

$$
D_{d}^{K}(P, Q) \geq D_{d}^{K}(M(P), M(Q)) .
$$

This completes the proof of the sufficiency of Eq. (71). The proof of the sufficiency of Eq. (72) follows in a similar manner, and we do not present it here.

\section{Appendix D: TRIANGLE INEQUALITY FOR THE EHS DISTANCE}

$$
\begin{aligned}
& \text { Let } \\
& \qquad D^{\mathrm{EHS}}(P, Q)= \\
& \Delta\left(\sum_{\rho, \sigma \in \Omega} P(\rho, \sigma) \rho \otimes[\rho \sigma], \sum_{\rho, \sigma \in \Omega} Q(\rho, \sigma) \sigma \otimes[\rho \sigma]\right)
\end{aligned}
$$

and

$$
\begin{gathered}
D^{\mathrm{EHS}}(Q, R)= \\
\Delta\left(\sum_{\kappa, \sigma \in \Omega} Q^{\prime}(\kappa, \sigma) \sigma \otimes[\kappa \sigma], \sum_{\kappa, \sigma \in \Omega} R^{\prime}(\kappa, \sigma) \kappa \otimes[\kappa \sigma]\right) .
\end{gathered}
$$

Here, the joint probability distributions $P(\rho, \sigma), Q(\rho, \sigma)$, $Q^{\prime}(\rho, \sigma), \quad R^{\prime}(\rho, \sigma)$ are such that the maxima for $D^{\mathrm{EHS}}(P, Q)$ and $D^{\mathrm{EHS}}(Q, R)$ in Eq. (82) are achieved. (The left marginals of $P(\rho, \sigma)$ and $R^{\prime}(\rho, \sigma)$ are $P(\rho)$ and $R(\rho)$, respectively, and the right marginals of $Q(\rho, \sigma)$ and $Q^{\prime}(\rho, \sigma)$ are equal to $Q(\sigma)$.)

Note that $Q(\rho, \sigma)$ and $Q^{\prime}(\rho, \sigma)$ are generally different, and we cannot use directly the triangle inequality of $\Delta$ to prove Eq. (95). This is why, we will construct two CPTP maps, $\mathcal{M}$ and $\mathcal{M}^{\prime}$, which map the states $\sum_{\rho, \sigma \in \Omega} Q(\rho, \sigma) \sigma \otimes[\rho \sigma]$ and $\sum_{\kappa, \sigma \in \Omega} Q^{\prime}(\kappa, \sigma) \sigma \otimes$ $[\kappa \sigma]$, respectively, to the same state, while at the same time transform the states $\sum_{\rho, \sigma \in \Omega} P(\rho, \sigma) \rho \otimes[\rho \sigma]$ and $\sum_{\kappa, \sigma \in \Omega} R^{\prime}(\kappa, \sigma) \kappa \otimes[\kappa \sigma]$, respectively, to valid EHS representations of the ensembles $P(\rho)$ and $R(\rho)$. Then using the monotonicity under CPTP maps of $\Delta$, it will follow that

$$
\begin{gathered}
D^{\mathrm{EHS}}(P, Q)+D^{\mathrm{EHS}}(Q, R) \geq \\
\Delta\left(\mathcal{M}\left(\sum_{\rho, \sigma \in \Omega} P(\rho, \sigma) \rho \otimes[\rho \sigma]\right), \mathcal{M}\left(\sum_{\rho, \sigma \in \Omega} Q(\rho, \sigma) \sigma \otimes[\rho \sigma]\right)\right)+ \\
\Delta\left(\mathcal{M}^{\prime}\left(\sum_{\kappa, \sigma \in \Omega} Q^{\prime}(\kappa, \sigma) \sigma \otimes[\kappa \sigma]\right), \mathcal{M}^{\prime}\left(\sum_{\kappa, \sigma \in \Omega} R^{\prime}(\kappa, \sigma) \kappa \otimes[\kappa \sigma]\right)\right) \\
\geq \Delta\left(\mathcal{M}\left(\sum_{\rho, \sigma \in \Omega} P(\rho, \sigma) \rho \otimes[\rho \sigma]\right), \mathcal{M}^{\prime}\left(\sum_{\kappa, \sigma \in \Omega} R^{\prime}(\kappa, \sigma) \kappa \otimes[\kappa \sigma]\right)\right) \\
=\Delta(\widehat{\rho}, \widehat{\kappa}) \geq D^{\mathrm{EHS}}(P, R),
\end{gathered}
$$

where $\widehat{\rho}$ and $\widehat{\kappa}$ are EHS representations of $P(\rho)$ and $R(\rho)$. What remains to be shown is that maps $\mathcal{M}$ and $\mathcal{M}^{\prime}$ with the above properties exist.

The maps that we propose act on the pointer space as follows:

$$
\begin{gathered}
\mathcal{M}([\rho \sigma])=T_{\sigma}(\kappa \mid \rho)[\kappa \rho \sigma], \\
\mathcal{M}^{\prime}([\kappa \sigma])=T_{\sigma}^{\prime}(\rho \mid \kappa)[\kappa \rho \sigma],
\end{gathered}
$$

where for every $\sigma, T_{\sigma}(\kappa \mid \rho)$ and $T_{\sigma}^{\prime}(\rho \mid \kappa)$ describe transition probabilities from $\rho$ to $\kappa$ and from $\kappa$ to $\rho$, respectively, such that

$$
T_{\sigma}(\kappa \mid \rho) Q(\rho, \sigma)=T_{\sigma}^{\prime}(\rho \mid \kappa) Q^{\prime}(\kappa, \sigma) \equiv J_{\sigma}(\kappa, \rho) .
$$

The fact that such transition probabilities exist follows from the fact that for every $\sigma, \sum_{\rho} Q(\rho, \sigma)=$ $\sum_{\kappa} Q^{\prime}(\kappa, \sigma)=Q(\sigma)$, i.e., for every fixed $\sigma, Q(\rho, \sigma)$ and $Q^{\prime}(\kappa, \sigma)$ describe (unnormalized) distributions of $\rho$ and $\kappa$ that have the same weight and therefore can be mapped one on top of each other via stochastic matrices that map $\rho$ to $\kappa$ or $\kappa$ to $\rho$.

By construction, we have

$$
\begin{gathered}
\mathcal{M}\left(\sum_{\rho, \sigma \in \Omega} Q(\rho, \sigma) \sigma \otimes[\rho \sigma]\right)=\mathcal{M}^{\prime}\left(\sum_{\kappa, \sigma \in \Omega} Q^{\prime}(\kappa, \sigma) \sigma \otimes[\kappa \sigma]\right) \\
=\sum_{\kappa, \rho, \sigma} J_{\sigma}(\kappa, \rho) \sigma \otimes[\kappa \rho \sigma] .
\end{gathered}
$$

Let us now verify that $\mathcal{M}$ and $\mathcal{M}^{\prime}$ applied to $\sum_{\rho, \sigma \in \Omega} P(\rho, \sigma) \rho \otimes[\rho \sigma]$ and $\sum_{\kappa, \sigma \in \Omega} R^{\prime}(\kappa, \sigma) \kappa \otimes[\kappa \sigma]$, respectively, give rise to valid EHS representations of $P$ and $R$. From the definition of the maps (D4) and (D5), one immediately obtains

$$
\begin{aligned}
& \mathcal{M}\left(\sum_{\rho, \sigma \in \Omega} P(\rho, \sigma) \rho \otimes[\rho \sigma]\right)= \\
& \sum_{\kappa, \rho, \sigma} T_{\sigma}(\kappa \mid \rho) P(\rho, \sigma) \rho \otimes[\kappa \rho \sigma]
\end{aligned}
$$

and

$$
\begin{gathered}
\mathcal{M}^{\prime}\left(\sum_{\kappa, \sigma \in \Omega} R^{\prime}(\kappa, \sigma) \kappa \otimes[\kappa \sigma]\right)= \\
\sum_{\kappa, \rho, \sigma} T_{\sigma}^{\prime}(\rho \mid \kappa) R^{\prime}(\kappa, \sigma) \kappa \otimes[\kappa \rho \sigma] .
\end{gathered}
$$

The fact that these are EHS representations of the ensembles $P$ and $R$ follows from two observations. The first one is that from the pointer $[\kappa \rho \sigma]$ one can unambiguously determine the state $\rho$ or $\kappa$ in the ensemble $P$ or $R$. The second one is that the joint probability distributions $T_{\sigma}(\kappa \mid \rho) P(\rho, \sigma)$ and $T_{\sigma}^{\prime}(\rho \mid \kappa) R^{\prime}(\kappa, \sigma)$ have the correct marginals,

$$
\begin{gathered}
\sum_{\kappa, \sigma} T_{\sigma}(\kappa \mid \rho) P(\rho, \sigma)= \\
\sum_{\sigma}\left(\sum_{\kappa} T_{\sigma}(\kappa \mid \rho)\right) P(\rho, \sigma)=\sum_{\sigma} P(\rho, \sigma)=P(\rho),
\end{gathered}
$$




$$
\begin{gathered}
\sum_{\rho, \sigma} T_{\sigma}^{\prime}(\rho \mid \kappa) R^{\prime}(\kappa, \sigma)= \\
\sum_{\sigma}\left(\sum_{\rho} T_{\sigma}^{\prime}(\rho \mid \kappa)\right) R^{\prime}(\kappa, \sigma)=\sum_{\sigma} R^{\prime}(\kappa, \sigma)=R(\kappa) .
\end{gathered}
$$

This completes the proof.

\section{Appendix E: CONTINUITY OF THE AVERAGE OF A CONTINUOUS FUNCTION WITH RESPECT TO THE EHS DISTANCE}

Let $h(\rho)$ be a bounded function, which is continuous with respect to the distance $\Delta$, i.e., for every $\delta>0$, there exists $\varepsilon>0$, such that for all $\rho$ and $\sigma$ for which

$$
\Delta(\rho, \sigma) \leq \varepsilon,
$$

we have

$$
|h(\rho)-h(\sigma)| \leq \frac{1}{2} \delta
$$

Let $\bar{h}_{P}$ denote the average of the function $h(\rho)$ over the ensemble $P(\rho), \rho \in \Omega$,

$$
\bar{h}_{P}=\sum_{\rho \in \Omega} P(\rho) h(\rho) .
$$

We will show that for every $\delta>0$, there exists $\varepsilon^{\prime}>0$, such that for all $P, Q \in \mathcal{P}_{\Omega}$ for which

$$
D^{\text {EHS }}(P, Q) \leq \varepsilon^{\prime},
$$

we have

$$
\left|\bar{h}_{P}-\bar{h}_{Q}\right| \leq \delta .
$$

Assume that $D^{\mathrm{EHS}}(P, Q) \leq \varepsilon^{\prime}$. Let $P(\rho, \sigma)$ and $Q(\rho, \sigma)$ be two joint distributions for which the minimum in Eq. (87) is attained. We then have

$$
D^{\mathrm{EHS}}(P, Q)=\frac{1}{2} \sum_{\rho, \sigma \in \Omega}\|P(\rho, \sigma) \rho-Q(\rho, \sigma) \sigma\| \leq \varepsilon^{\prime} .
$$

Define the sets $\Omega_{>\varepsilon}$ and $\Omega_{\leq \varepsilon}$ as the sets of all pairs of states $(\rho, \sigma)$ for which $\Delta(\rho, \sigma)>\varepsilon$ and $\Delta(\rho, \sigma) \leq \varepsilon$, respectively. The sum in Eq. (E6) can then be split in two sums,

$$
\begin{gathered}
\frac{1}{2} \sum_{\Omega_{>\varepsilon}}\|P(\rho, \sigma) \rho-Q(\rho, \sigma) \sigma\|+ \\
\frac{1}{2} \sum_{\Omega_{\leq \varepsilon}}\|P(\rho, \sigma) \rho-Q(\rho, \sigma) \sigma\| \leq \varepsilon^{\prime} .
\end{gathered}
$$

The first sum obviously can be bounded from above as

$$
\frac{1}{2} \sum_{\Omega_{>\varepsilon}}\|P(\rho, \sigma) \rho-Q(\rho, \sigma) \sigma\| \leq \varepsilon^{\prime} .
$$

Notice also that since the trace distance is monotonic under tracing, we have

$$
\begin{gathered}
\frac{1}{2} \sum_{\rho, \sigma \in \Omega}|P(\rho, \sigma)-Q(\rho, \sigma)| \leq \\
\frac{1}{2} \sum_{\rho, \sigma \in \Omega}\|P(\rho, \sigma) \rho-Q(\rho, \sigma) \sigma\| \leq \varepsilon^{\prime} .
\end{gathered}
$$

Therefore,

$$
\frac{1}{2} \sum_{\Omega_{>\varepsilon}}|P(\rho, \sigma)-Q(\rho, \sigma)| \leq \varepsilon^{\prime},
$$

and

$$
\frac{1}{2} \sum_{\Omega_{\leq \varepsilon}}|P(\rho, \sigma)-Q(\rho, \sigma)| \leq \varepsilon^{\prime} .
$$

On the other hand, we have

$$
\begin{gathered}
\sum_{\Omega_{>\varepsilon}} P(\rho, \sigma) \varepsilon \leq \frac{1}{2} \sum_{\Omega_{>\varepsilon}} P(\rho, \sigma)\|\rho-\sigma\| \leq \\
\frac{1}{2} \sum_{\Omega_{>\varepsilon}}\|P(\rho, \sigma) \rho-Q(\rho, \sigma) \sigma\|+\frac{1}{2} \sum_{\Omega_{>\varepsilon}}|Q(\rho, \sigma)-P(\rho, \sigma)| \\
\leq \varepsilon^{\prime}+\varepsilon^{\prime}=2 \varepsilon^{\prime}, \quad \text { (E12) }
\end{gathered}
$$

where the second inequality follows from the triangle inequality for the trace distance and the third inequality follows from Eqs. (E8) and (E10). This implies

$$
\sum_{\Omega_{>\varepsilon}} P(\rho, \sigma) \leq \frac{2 \varepsilon^{\prime}}{\varepsilon}
$$

Let us now look at the difference between the average functions over the two ensembles.

$$
\begin{gathered}
\left|\bar{h}_{P}-\bar{h}_{Q}\right|=\left|\sum_{\rho \in \Omega} P(\rho) h(\rho)-\sum_{\sigma \in \Omega} Q(\sigma) h(\sigma)\right| \\
=\left|\sum_{\rho, \sigma \in \Omega} P(\rho, \sigma) h(\rho)-\sum_{\rho, \sigma \in \Omega} Q(\rho, \sigma) h(\sigma)\right| \\
\leq \sum_{\rho, \sigma \in \Omega}|P(\rho, \sigma) h(\rho)-Q(\rho, \sigma) h(\sigma)| \leq \\
\sum_{\rho, \sigma \in \Omega}(P(\rho, \sigma)|h(\rho)-h(\sigma)|+|Q(\rho, \sigma)-P(\rho, \sigma)||h(\sigma)| \\
=\sum_{\Omega_{>\varepsilon}} P(\rho, \sigma)|h(\rho)-h(\sigma)|+\sum_{\Omega_{\leq \varepsilon}} P(\rho, \sigma)|h(\rho)-h(\sigma)|+ \\
\sum_{\rho, \sigma \in \Omega}|Q(\rho, \sigma)-P(\rho, \sigma)||h(\sigma)| .
\end{gathered}
$$

Since $h(\rho)$ is bounded, there exists a constant $h_{\max }>0$ such that $|h(\rho)-h(\sigma)| \leq h_{\max }$ and $|h(\rho)| \leq h_{\max }$ for all $\rho$ and $\sigma$. Using this fact, together with Eqs. (E13) and (E9) and the assumption that for all $(\rho, \sigma) \in \Omega_{\leq \varepsilon}$, 
$|h(\rho)-h(\sigma)| \leq \frac{1}{2} \delta$, we can upper bound the last line in Eq. (E14) as follows:

$$
\begin{gathered}
\sum_{\Omega_{>\varepsilon}} P(\rho, \sigma)|h(\rho)-h(\sigma)|+\sum_{\Omega_{\leq \varepsilon}} P(\rho, \sigma)|h(\rho)-h(\sigma)|+ \\
\sum_{\rho, \sigma \in \Omega}|Q(\rho, \sigma)-P(\rho, \sigma)||h(\sigma)| \leq \\
\frac{2 \varepsilon^{\prime}}{\varepsilon} h_{\max }+\sum_{\Omega_{\leq \varepsilon}} P(\rho, \sigma) \frac{1}{2} \delta+2 \varepsilon^{\prime} h_{\max } \leq \\
\frac{2 \varepsilon^{\prime}}{\varepsilon} h_{\max }+\frac{1}{2} \delta+2 \varepsilon^{\prime} h_{\max } .
\end{gathered}
$$

Therefore, we see that by choosing

$$
\varepsilon^{\prime} \leq \frac{\delta \varepsilon}{4 h_{\max }(1+\varepsilon)},
$$

we obtain

$$
\left|\bar{h}_{P}-\bar{h}_{Q}\right| \leq \delta
$$

Since $\delta$ was arbitrarily chosen, the property follows.

\section{ACKNOWLEDGMENTS}

The authors thank Emili Bagan, Ramón Muñoz-Tapia, Oriol Romero-Isart, Igor Devetak and Nathan K. Langford for helpful discussions. This work was supported by the Spanish MICINN through the Ramón y Cajal program (JC), contract FIS2008-01236/FIS, and project QOIT (CONSOLIDER2006-00019), and by the Generalitat de Catalunya through CIRIT 2005SGR-00994.
[1] A. Uhlmann, Rep. Math. Phys. 9, 273 (1976).

[2] M. Nielsen and I. Chuang, Quantum Computation and Quantum Information (Cambridge Univeristy Press, Cambridge, 2000).

[3] D. Bures, Trans. Am. Math. Soc. 135, 199 (1969).

[4] M. Hübner, Phys. Lett. A 163, 239 (1992).

[5] A. Uhlmann, in Quantum Groups and Related Topics. Proceedings of the First Max Born Symposium, (edited by R. Gielerak, J. Lukierski, and Z. Popovicz), pp. 267 274 (Kluwer Acad. Publishers, 1992).

[6] C.W. Helstrom, Quantum Detection and Estimation Theory (Academic, New York, 1976).

[7] J. Lee, M. S. Kim, and C. Brukner, Phys. Rev. Lett. 91, 087902 (2003).

[8] K. M. R. Audenaert, J. Calsamiglia, R. Munoz-Tapia, E. Bagan, Ll. Masanes, A. Acin, and F. Verstraete, Phys. Rev. Lett. 98, 160501 (2007).

[9] J. Calsamiglia, R. Munoz-Tapia, Ll. Masanes, A. Acin, and E. Bagan, Phys. Rev. A 77, 032311 (2008).

[10] O. Oreshkov, Phys. Rev. A 77, 032333 (2008).

[11] I. Bengtsson and K. Życzkowski, Geometry of Quantum States: An Introduction to Quantum Entanglement (Cambridge Univeristy Press, Cambridge, 2006).

[12] C. A. Fuchs, Ph.D. thesis, University of New Mexico, Albaquerque, NM, 1996, e-print arXiv:quant-ph/9601020

[13] S. L. Braunstein, C. A. Fuchs, and H. J. Kimble, J. Mod. Opt. 47, 267 (2000).

[14] G. Gour and R. W. Spekkens, New J. Phys. 10, 033023 (2008).

[15] G. Vidal, J. Mod. Opt. 47, 255 (2000).

[16] O. Oreshkov and T. A. Brun, Phys. Rev. A 73, 042314 (2006).

[17] L. B. Levitin, "On the quantum measure of the amount of information", in Proceedings of the Fourth All-Union Conference on Information Theory, Tashkent (1969), pp. 111, in Russian.

[18] A. S. Holevo, "Bounds for the quantity of information transmitted by a quantum communication channel", Probl. Peredachi Inf. 9(3), 311 (1973) [in Russian; English translation in Probl. Inf. Transm. (USSR) 9, 177183 (1973)].
[19] I. Devetak and A. Winter, e-print arXiv:quant-ph/0304196 (2003).

[20] A. Gilchrist, N. K. Langford, and M. A. Nielsen, Phys. Rev. A 71, 062310 (2005).

[21] C. H. Bennett, H. J. Bernstein, S. Popescu, B. Schumacher, Phys. Rev. A 532046 (1996).

[22] D. Gottesman, Stabilizer codes and quantum error orrection, Ph.D. thesis, Caltech, 1997, e-print arXiv:quant-ph/9705052 (1997).

[23] A. Luis and L. L. Sanchez-Soto, Phys. Rev. Lett. 83, 3573 (1999).

[24] J. Fiurasek, Phys. Rev. A 64, 024102 (2001).

[25] G. M. D'Ariano, L. Maccone, and P. Lo Presti, Phys. Rev. Lett. 93, 250407 (2004).

[26] J. S. Lundeen, A. Feito, H. Coldenstrodt-Ronge, K. L. Pregnell, Ch. Silberhorn, T. C. Ralph, J. Eisert, M. B. Plenio and I. A. Walmsley, Nature Phys. 5, 27 (2009).

[27] A. Jamiołkowski, Rep. Math. Phys. 3, 275 (1972).

[28] L. V. Kantorovich, Dokl. Akad. Nauk SSSR, 37, No. 78, 227229 (1942).

[29] C. Villani, Optimal Transport: Old and New (Spinger, Berlin, 2009)

[30] L. N. Vasershtein, "Markov processes on a countable product space, describing large systems of automata", Problemy Peredachi Informatsii, 5, 3 (1969), pp. 64-73.

[31] K. Kraus, States, Effects, and Operations: Fundamental Notions of Quantum Theory, Lecture Notes in Physics Vol. 190 (Springer-Verlag, Berlin, 1983).

[32] M. B. Ruskai, Rev. Math. Phys. 6, 1147 (1994).

[33] A. S. Holevo, IEEE Trans. Info. Theory 44, 269 (1998).

[34] B. Schumacher and M. D. Westmoreland, Phys. Rev. A 56, 131 (1997).

[35] K. M. R. Audenaert, J. Phys. A: Math. Theor. 40, 8127 (2007).

[36] M. Fannes, Commun. Math. Phys. 31, 291 (1973).

[37] R. Jozsa, J. Mod. Opt. 41, 2315 (1994).

[38] J. L. Dodd and M. A. Nielsen, e-print arXiv:quant-ph/0111053 (2001).

[39] D. Aharonov, A. Kitaev, and N. Nisan, in Proceedings of the 30th Annual ACM Symposium on Theory of Computation (STOC), pp. 20-30 (1998). 
[40] H.-J. Sommers, and K. Życzkowski, J. Phys. A: Math. Gen. 36, 10083 (2003).

[41] I. L. Chuang and M. A. Nielsen, J. Mod. Opt. 44, 2455 (1997).
[42] J. F. Poyatos, J. I. Cirac, and P. Zoller, Phys. Rev. Lett. 78, 390 (1997). 